UNIVERSIDADE DE BRASÍLIA - UnB INSTITUTO DE GEOCIÊNCIAS - IG

PROGRAMA DE PÓS-GRADUAÇÃO EM GEOLOGIA

\title{
SISMOESTRATIGRAFIA DOS DEPÓSITOS CENOZÓICOS DA BACIA SALAVERRY, PORÇÃO OFFSHORE DO PERU: NOVAS INTERPRETAÇÕES DO SISTEMA PETROLÍFERO
}

DISSERTAÇÃO DE MESTRADO No 356

Diego Fernando Timoteo Martinez

Brasília, DF, Dezembro de 2015 
UNIVERSIDADE DE BRASÍLIA - UnB INSTITUTO DE GEOCIÊNCIAS - IG

PROGRAMA DE PÓS-GRADUAÇÃO EM GEOLOGIA

\section{SISMOESTRATIGRAFIA DOS DEPÓSITOS CENOZÓICOS DA BACIA SALAVERRY, PORÇÃO OFFSHORE DO PERU: NOVAS INTERPRETAÇÕES DO SISTEMA PETROLÍFERO}

Diego Fernando Timoteo Martinez

Orientador:

Prof. Dr. Farid Chemale Junior

Banca Examinadora:

Prof. Dr. Farid Chemale Junior (UnB)

Prof. Dr. Carlos Emanoel de Souza Cruz (UnB)

Prof. Dr. Paulo Sergio Gomes Paim (Unisinos)

Brasília, DF, Dezembro de 2015 
Este trabalho está dedicado a meu pai Fernando, minha mãe Edicta, minha irmã Evelyn e minha sobrinha Camila, como retribuição por todo o amor e apoio que recebo sempre deles. 


\section{RESUMO}

O presente trabalho aborda o preenchimento sedimentar e estrutura da Bacia Salaverry que se formou durante o Cenozóico na porção central da margem ativa do Peru, especificamente na região defronte o Arco Magmático Peruano dos Andes. A Bacia Salaverry é importante por conter três "oil seeps" amostrados e analisados, os quais sugerem a presença de pelo menos um sistema petrolífero ativo no interior desta. Por meio da análise de dados sísmicos com a calibração de dados de subsuperfície obtidos em poços exploratórios e dados de termocronologia por traços de fissão em apatita, pode-se estabelecer a evolução tectono-sedimentar dos sistemas deposicionais cenozóicos desta bacia. A sedimentação desta bacia foi controlada por processos extensionais com tectônica compressiva localizada, os quais estão relacionados à interação das placas de Nazca e Sul-americana e a subducção da dorsal de Nazca. A sucessão Cenozóica na porção central da Bacia Salaverry foi, portanto, subdividida em onze sequências sismoestratigráficas (S1-S11), as quais tiveram controle tectônico importante e também flutuação do nível do mar. Com base ao sistema petrolífero já caracterizado na bacia, novos reservatórios potenciais são definidos nas S1, S2, S4, S5, S7 e S8, enquanto rochas selantes foram identificadas nas sequencias S3, S6, S7 e S9S11. Análises termocronológicas por traços de fissão em apatita permitiram definir no registro da bacia um evento de soerguimento principal em torno de $25 \mathrm{Ma}$, correlacionado à fase tectônica Incaica III, que resultou em erosão das rochas oligocênicas e parte superior dos depósitos eocênicos superiores. Estes novos elementos do sistema petrolíferos tornam a porção central da Bacia Salaverry mais atrativa para atividades exploratórias futuras.

Palavras-chave: Bacia Salaverry, Sismoestratigrafia, Exploração de hidrocarbonetos, Plays, Bacias de forearc, Margem Peruana, Andes. 


\section{ABSTRACT}

This study is focused in Salaverry Basin, Peruvian Andes Forearc, which contains three oil seeps sampled and analyzed, suggesting at least one active petroleum system inside the basin. Seismic stratigraphic analysis calibrated with available well and core data, and integrated with Apatite fission track (AFT) analysis was performed in order to provide the spatial-temporal evolution of Cenozoic depositional systems in this basin. It has been developed in extensional tectonic setting punctuated by compressive periods controlled by the interaction between Nazca and South American plates, and Nazca ridge subduction. As a result, the Cenozoic succession in the central portion of Salaverry Basin is best subdivided into eleven seismic stratigraphic sequences (S1S11), controlled predominantly by tectonism and also by sea-level fluctuations. Indeed, this work adds new potential reservoir rocks in the S1, S2, S4, S5, S7 and S8 sequences; and seal rocks in the S3, S6, S7 and S9-S11 ones for the petroleum systems previously defined in the basin. Fission track thermochronology analysis on apatite suggest a main uplift event in the basin at ca. $25 \mathrm{Ma}$, which triggered the erosion of Oligocene rocks and the uppermost section of the Upper Eocene deposits, and is correlated with the Incaic III tectonic phase. These new petroleum system elements are related to the proposed potential plays, which in turn through a follow-up assessment would become in very attractive exploratory prospects in Salaverry Basin central portion.

Keywords: Salaverry Basin, Seismic stratigraphy, Hydrocarbon exploration, Plays, Forearc Basin, Peruvian margin, Andes. 


\section{SUMÁRIO}

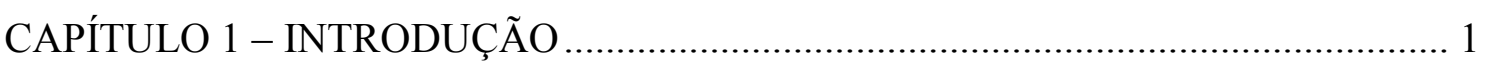

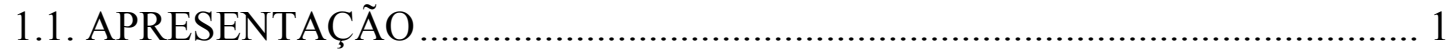

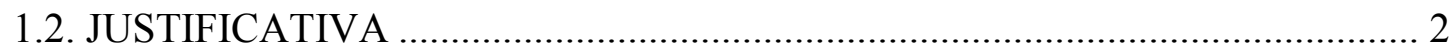

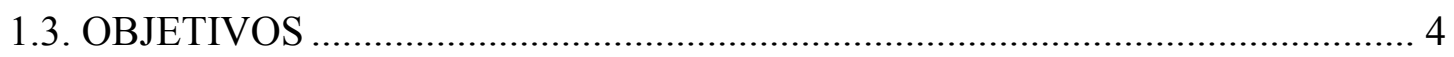

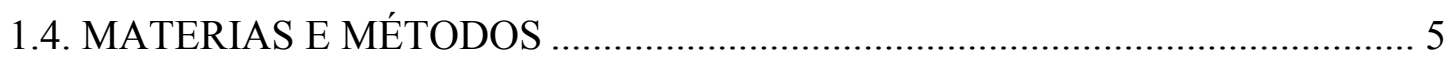

CAPÍTULO 2 - CONTEXTO GEOLÓGICO …………………………………….... 9

2.1. MECANISMOS QUE CONTROLAM A REGIÃO DEFRONTE AO ARCO .... 9

2.2. CICLO OROGÊNICO ANDINO …………………………………………..... 15

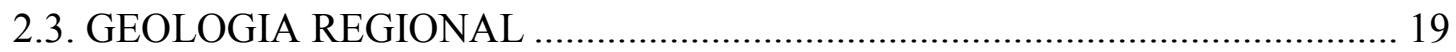

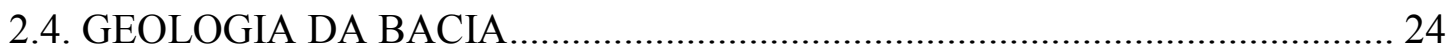

CAPÍTULO 3: TECTONO-SEDIMENTARY EVOLUTION OF THE SALAVERRY BASIN, PERUVIAN ANDES FOREARC: NEW INSIGHTS IN ASSESSING POTENTIAL RESERVOIRS AND PLAYS ......................................................... 39

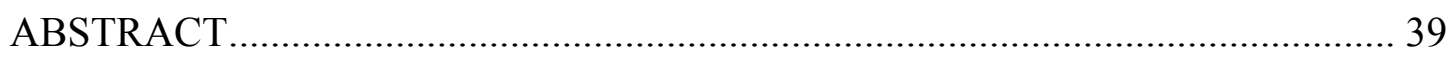

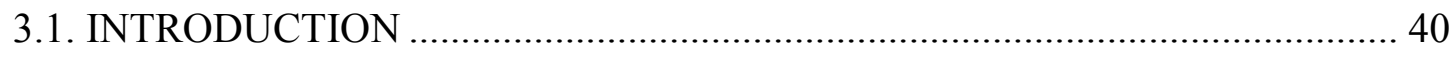

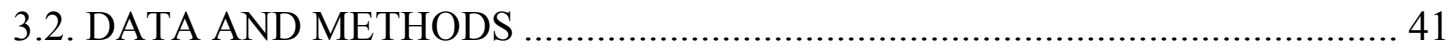

3.2.1. SEISMIC AND WELL LOG DATA......................................................... 41

3.2.2. THERMOCRONOLOGICAL ANALYSIS ……………………………...... 43

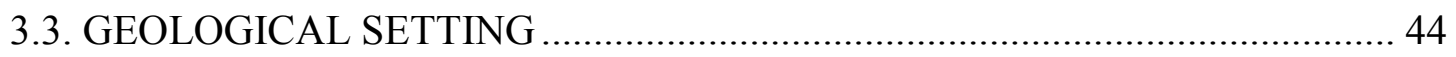

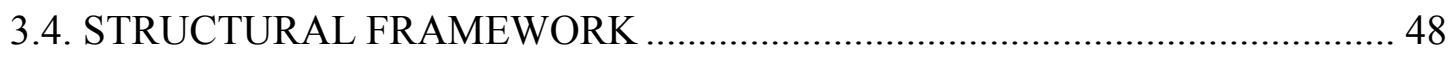

3.5. SEISMIC STRATIGRAPHY OF THE CENOZOIC SUCCESSION.................. 49

3.6. RESULTS OF THERMOCHRONOLOGICAL ANALYSIS ............................... 68

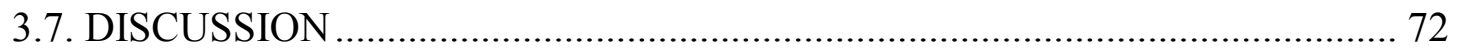

3.7.1. TECTONO-SEDIMENTARY EVOLUTION_............................................ 72

3.7.2. NEW PETROLEUM SYSTEM INSIGHTS ……………………………..... 76

3.7.2.1. HYDROCARBON EVIDENCES ………………………………........ 76

3.7.2.2. POTENTIAL RESERVOIR AND SEAL ROCKS ................................. 78

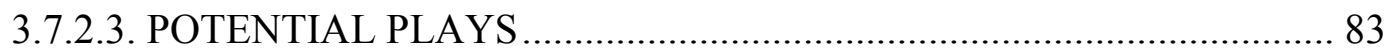

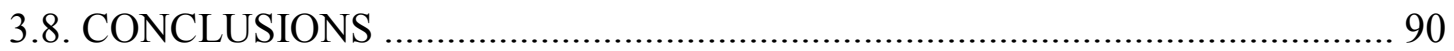

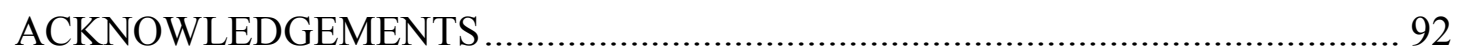

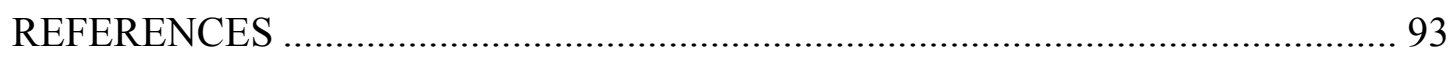


CAPÍTULO 4: CONCLUSÕES

97

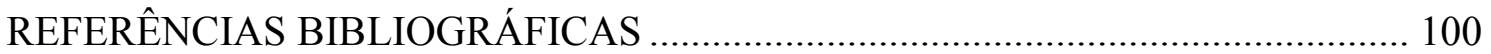

ANEXO 1: Carta de submissão do Artigo …………………………………….... 108 


\section{LISTA DE FIGURAS}

Figura 1. Mapa de localização da Bacia Salaverry (delineada por tracejado amarelo) no segmento de bacias de "fore-arc" (defronte ao arco) prolíferas em hidrocarbonetos no Peru. Observa-se também a indicação de volume em milhões de barris de petróleo (MMBP) nas Bacias Sechura e Talara, poços e oil seeps (Modificado de Timoteo et al., 2014)

\section{.2}

Figura 2. Mapa de localização da área de estudo na porção centro-norte da Bacia Salaverry com a disposição dos dados sísmicos 2D e 3D utilizados no projeto, na seção offshore, assim como as principais unidades estratigráficas aflorantes na margem continental emersa adjacente (onshore).

Figura 3. Modelos conceituais nas margens de tipo Andino: (a) margem compressiva, que é a configuração dominante na maior parte dos Andes hoje em dia; (b) margem trativa como vista na parte norte de America Central (Segundo Ramos, 2010). .9

Figura 4. Relação entre os eventos tectônicos, a taxa de convergência (ou velocidade de convergência) e a idade da placa oceânica durante a subducção na margem oeste de America do Sul (modificado de Jaillard et al., 2000 em Wipf, 2006). .11

Figura 5. Principais parâmetros cinemáticos que controlam o regime tectônico em uma margem do tipo Andino. Vt: velocidade de retrocesso da trincheira; Vup: movimento absoluto da placa superior; Vsh: taxa de encurtamento; Vst: taxa de alongamento e Ver: taxa de erosão crustal (Segundo Ramos, 2010).

Figura 6. Principais cenários na configuração Andina atual: (a) velocidade de retrocesso da trincheira igual a zero; (b) velocidade de retrocesso da trincheira negativa $(\mathrm{Vt}<0)$ na margem tipo Marianas; (c) velocidade de retrocesso da trincheira positiva $(\mathrm{Vt}>0)$ na margem tipo Peru-Chile (Segundo Ramos, 2010). .13

Figura 7. Evolução tectônica idealizada dum Ciclo Orogênico Andino. L: limite litosfera-astenosfera; ML: manto litosférico (Segundo Ramos, 2009b)...

Figura 8. Configuração tectônica da Placa Nazca e sua relação com a área de estudo, a qual está situada na margem oeste da Placa Sul-americana. Estão inseridas as principais feições morfo-estruturais, que influenciam a margem continental peruana, e a idade da placa oceânica (modificado de Lajo, 2014)

Figura 9. Mapa com distribuição do embasamento nos Andes Peruanos e sua relação com as Deflexões de Huancabamba e Abancay. A sutura oriental (paleosutura) indica a protomargem de Gondwana no Perú (modificado de Ramos, 2014). Na região offshore observa-se o OSH que é evidenciado nas rochas expostas nas ilhas e nas rochas metamórficas perfuradas nos poços exploratórios (modificado de Romero et al., 2013) 
Figura 10. Trend NW-SE das bacias de fore-arc Peruanas, limitada a oeste pelo alto da plataforma externa (Outer Shelf High). O mapa estrutural do topo do embasamento PréCambriano-Paleozóico é baseado em interpretação sísmica, enquanto que as cadeias descontinuas de altos de embasamento são baseadas na interpretação de dados gravimétricos e magnetométricos (Romero et al., 2013).

Figura 11. A) Vista da Ilha Las Hormigas de Afuera, onde a amostragem do gnaisse foi realizada. B) Resumo das principais idades dos zircões obtidos no gnaisse: Famatiniano, Grenvilliano, e alguns zircões herdados paleoproterozóicos. Localização da ilha na Figura 9 (Modificado de Romero et al., 2013). .25

Figura 12. Gnaisse perfurado pelo poço exploratório Ballena-1X: A) microfotografia da lamina delgada em nicóis paralelos e B) microfotografia da lamina delgada em nicóis cruzados. Localização do poço na Figura 9

Figura 13. A) Microfotografia da lâmina delgada em nicóis cruzados do diorito perfurado pelo poço exploratório San Miguel-1X. B) Idade Ar-Ar do diorito San Miguel. Localização do poço na Figura 9 (Modificado de Timoteo et al., 2012) .26

Figura 14. A) Afloramentos da Formação La Leche do Triássico superior-Jurássico inferior, no Vale do Rio La Leche, ao norte da área de estudo. B) Ampliação da foto anterior onde se mostram calcários cinza escuros na base da secção.

Figura 15. A) Vista panorâmica dos afloramentos da Formação Chicama do Jurássico superior, no Vale do Rio Chicama. B) Folhelhos cinza escuros laminados que conformam os depósitos turbidíticos na seção basal da Formação Chicama (Modificado de Timoteo, 2013). .28

Figura 16. A) Microfotografia da lâmina delgada em nicóis cruzados do basalto amostrado na Ilha Macabi. B) Zircões identificados no basalto e separados para datação U-Pb TIMS. C) Concórdia das idades dos zircões datados no basalto. D) Ampliação da concórdia, onde se observa a idade máxima que poderia atingir o basalto da Ilha Macabi. Localização da ilha na Figura 9 (modificado de Timoteo et al., 2012) .28

Figura 17. A) Arenitos com estratificação cruzada de grande porte pertencentes ao Grupo Goyllarisquizga na localidade de Simbron. B) Arenitos quartzosos fortemente fraturados pertencentes ao Grupo Goyllarisquizga. C) Afloramentos de folhelhos da Formação La Zorra, Praia La Zorra. D) Folhelhos pretos carbonosos reconhecidos como rocha geradora potencial em toda a secção estratigráfica da Fm. La Zorra. E) Afloramento de calcários do Grupo Pulluicana-Quilquiñan na localidade de Saltur. F) Calcários cinzas escuros fraturados, reconhecidos no topo da secção do Grupo Pulluicana-Quilquiñan (modificado de Timoteo et al., 2013). 
Figura 18. Mapa geológico simplificado do Peru com a disposição da Bacia Salaverry (delineada por tracejado vermelho) e a área de estudo (polígono preto), assim como a localização de afloramentos chaves

Figura 19. Perfil composto do poço exploratório Delfin-1X integrado com os dados de paleobatimetria .33

Figura 20. Perfil composto do poço exploratório Ballena-1X integrado com os dados de paleobatimetria.

Figura 21. Vista panorâmica e de detalhe dos afloramentos de idade PliocenoHoloceno reconhecidos no trabalho de campo. A) e B) Depósitos fuvio-aluviais que se sobrepõem discordantes às rochas vulcânicas do Cretácico superior. C) Depósitos de antepraia e praia. D) arenitos com estratificação cruzada tangencial. E) e F) Depósitos de leques aluviais. G) e H) Depósitos eólicos que se sobrepõem discordante aos granitos do Batólito Costeiro

Figure 1. Location map of the Peruvian Forearc petroliferous/tectonic province, comprising the Salaverry Basin (yellow dotted line) and Northwest petroliferous province (Talara and Sechura Basins). The exploratory wells and oil seeps from southcentral province are also indicated (Modified from Timoteo et al., 2014)

Figure 2. Map of the northern central Peruvian margin showing the location of the study area (green polygon) and its relation with the main fluvial system. The figure also illustrates the location of 2D and 3D seismic surveys, and the exploratory wells used in the present project

Figure 3. Time structure map of the Top Pre-Cenozoic Basement showing the main fault systems and the morphostructural domains in the study area. Contour lines are every $50 \mathrm{~ms} . \quad \mathrm{TB}=$ Trujillo Basin; $\mathrm{PA}=$ Paracas Arch; $\mathrm{CSB}=$ Cenozoic Salaverry Basin; VCBD $=$ Volcanic arc and Coastal Batolith Domain, SWD = Southwestern Depocentre. Also it illustrates the onshore geological settings....

Figure 4. Litostratigraphic subdivisions of the Cenozoic succession in the central portion of Salaverry Basin and the corresponding seismostratigraphic units interpreted in this study....

Figure 5. NE-SW Seismic sections of Salaverry Basin with interpreted Cenozoic seismic sequences $\mathrm{S} 1$ to $\mathrm{S} 11$. Seismic section locations in Figure 3. .51

Figure 6. NW-SW Seismic sections of Salaverry Basin with interpreted Cenozoic seismic sequences $\mathrm{S} 1$ to S11. Seismic section locations in Figure 3.... 52

Figure 7. Reflection configuration and terminations within the Cenozoic seismic sequences. A) Uninterpreted and B) interpreted 2D seismic section across the western margin of the basin over the Paracas Arch. The location of the seismic section is indicated in Figure 5B .56 
Figure 8. Isochron maps of the seismic stratigraphic sequences S1 to S5. Isochron maps depict the two way time (TWT) thickness calculated between top and bottom sequence boundary of each seismic stratigraphic sequence. White areas within the study area (black polygon) depict absence of the sequence or lack seismic interpretation due to data gaps or poor seismic quality. .58

Figure 9. Isochron maps of the seismic stratigraphic sequences S6 to

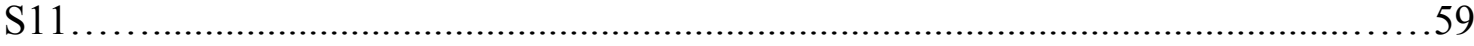

Figure 10. Seismic facies maps of the seismic stratigraphic sequences S1 to S5. Number of seismic facies in the study area (black polygon) increases from the S1 to the S11 sequences indicating that different depositional processes occurred during their deposition. Refer to Figure 3 for position of the basin and structural highs. See text for further explanation.

Figure 11. Seismic facies maps of the seismic stratigraphic sequences S6 to S11. See Table 1 for seismic facies description. .62

Figure 12. A) Uninterpreted and B) interpreted NE-SW seismic profile across the southern Paracas Arch. Note the thinning and absence of the S2 seismic sequence on the flanks and top of the structural high, respectively. C) Uninterpreted and D) interpreted NE-SW seismic profile across a intrabasinal high, where S3 sequence onlaps the SB1 seismic horizon. E) Uninterpreted and F) interpreted NE-SW seismic profile across the basin foredeep. Note the thinning and onlapping of S3 sequence towards the intrabasinal high flanks. The location of the seismic profiles is indicated in Figure $3 \ldots \ldots \ldots \ldots \ldots . .64$

Figure 13. Reflection configuration and terminations within the Cenozoic seismic sequences. A) Uninterpreted and B) interpreted 2D seismic section across the eastern margin of the basin. The location of the seismic section is indicated in Figure $5 \mathrm{~B}$

Figure 14. A) Uninterpreted and B) interpreted NE-SW seismic profile across the eastern margin, showing S4 to S9 sequences truncation against shallow structural high that depicts volcanic/intrusive bodies. The location of the seismic profile is indicated in Figure 3

Figure 15. A) Uninterpreted and B) interpreted dip seismic profile across the Cenozoic Salaverry Basin, showing the depositional strike of major delta within the S8 seismic sequence. C) Uninterpreted and D) interpreted strike seismic profile along the basin foredeep, showing the depositional dip of major delta within the S8 seismic sequence. Note the concave upward prograding clinoforms that conform the delta. The location of the seismic profile is indicated in Figures 3 and $6 \mathrm{E}$ 
Figure 16. Left column: radial plots showing single grain AFT age in detrital samples B1 and B7. Right column: thermal inverse modeling results of AFT ages from B1 and B7 samples of Ballena-1X exploratory well. The models were obtained with the HeFTy program (Ketcham, 2005) (Blue line: average of the good-fit thermal history. Magenta area: statistically good fit paths. Green area: acceptable fit

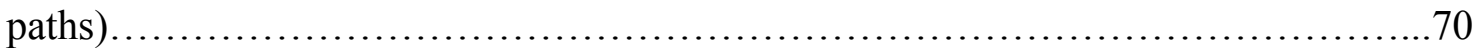

Figure 17. Sequence stratigraphic framework correlated with the Andean tectonic phases that illustrate the geological evolution of the study area. *Age of Incaic III tectonic phase is constrained by the apatite FT thermochronological data. .74

Figure 18. Seismic profiles showing different type of DHIs recognized in the study area. A) Potential shallow gas accumulations marked by bright spots and enhanced reflections. B) Fluid flow systems recognized as gas chimneys. C) and D) Leakage along faults, interpreted as pathways for deep fluid migration in the basin. ... .77

Figure 19. Core lithofacies from Ballena-1X exploratory well. A) Favorable reservoir facies within the Upper Eocene Verdun formation (S1 sequence) are described. B) Interbedded sandstones showing better conditions to potential reservoir rocks within the Lower Miocene Heath Formation (S2 sequence) are described .............................................................. 79

Figure 20. Seismic attribute maps from 3D seismic time slices in the study area: A) Amplitude and B) variance slice showing submarine channel system within S4. C) Variance and D) RMS amplitude slice, which better define another submarine channel system within S5. E) Amplitude and F) variance slices showing carbonate reefs within S7. G) Amplitude and H) RMS amplitude slice showing turbidite lobe at clinoform bottomsets from S8. I) Amplitude and J) variance slice showing deltaic/shoreface deposits at clinoform tops (offlap break) from S8. Refer to Figure 2 for 3D seismic cube location. .82

Figure 21. The colored polygons shown offshore represent the mean areal extent of each potential play identified in the central portion of Salaverry Basin within the study area. The bathymetry related to each potential play is shown in grayscale .85

Figure. 22. Seismic profiles showing many of the most relevant trap types recognized in the study area. A) onlaps and subunconformity truncations. B) Partially inverted normal fault, onlaps and toplaps. C) Embedded turbidite lobes encased in shales. D) Fault propagation folds. E) Normal faulted blocks. F) Faulted Roll-over anticline. G) Faulted Roll-over anticline related to major listric faults. Refer to Figure 21 for seismic profile locations. 


\section{LISTA DE TABELAS}

Table 1. Seismic Facies identified in the Cenozoic succession of Salaverry

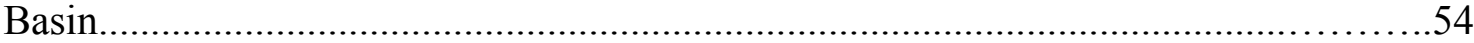

Table 2. Apatite fission track data from detrital samples belongs to Ballena-1X exploratory well from Salaverry Basin, Offshore Central Peru. 


\section{CAPÍTULO 1: INTRODUÇÃO}

\subsection{APRESENTAÇÃO}

A Bacia Salaverry localiza-se na porção de fore-arc dos Andes Peruanos, margem ativa do Perú central, especificamente em frente às costas de Chiclayo e Huacho e imediatamente ao sul das Bacias Sechura e Talara. Estas possuem produção de petróleo e gás importantes, das quais a Bacia Talara tem a maior produção acumulada que totalizam 2 bilhões de barris de petróleo enquanto a Bacia Sechura tem uma produção acumulada de 300 milhoes de barris de petróleo. Na porção centro-norte da Bacia de Salaverry, ao sul da Bacia de Talara, tem somente dois poços exploratórios e ocorrência de petróleo e gás comprovada, o que necessita uma investigação mais detalhada para se avaliar o seu potencial petrolífero (Figura 1).

O esforço exploratório na região contabiliza o poço Ballena-1X, perfurado na borda oeste de Bacia Salaverry e sem evidencias de hidrocarbonetos, o poço Delfin-1X perfurado na borda leste da bacia Trujillo e evidencias de gas shows. Além disso, a identificação de ocorrências de petróleo nos poços exploratórios Morsa-1X e Lobos1X, perfurados na região centro-norte da Bacia Trujillo, revelam a existência de pelo menos um sistema petrolífero ativo na periferia da bacia Salaverry (Quesada et al., 2000). A recente identificação de três oil seeps marinhos na Bacia Salaverry é também uma prova verídica do hidrocarboneto gerado dentro da mesma (Figura 1, Valencia et al., 2011; Valencia et al., 2013 e Timoteo et al., 2014).

Nos últimos anos, o incremento do conhecimento geológico na Bacia Salaverry permitiu delinear leads e prospectos exploratórios de interesse econômico por estar perto de cidades desenvolvidas ao longo da costa do Perú central, o que levou grandes empresas transnacionais concorrerem no ultimo bidding round, em 2013, realizado pela agencia nacional de petróleo - PERUPETRO (web 1, web 2).

Além dos plays estruturais Paleozóicos e Cretácicos reconhecidos pela empresa SAVIA PERU S.A., as sequências sedimentares cenozóicas representam atualmente uma nova frente exploratória, ainda que careça de estudos sismoestratigráficos de maior detalhe no presente conjunto de dados sísmicos da bacia. 
Assim, o presente trabalho tem como estratégia a reavaliação dos dados sísmicos e poucos dados de poços disponíveis sobre a Bacia Salaverry para: (i) definir melhor as sequências sismo-estratigráficas desta bacia e sua relação com os sistemas deposicionais e processos tectônicos andinos; (ii) caracterizar com maior detalhe os prováveis reservatórios de óleo e gás potenciais na bacia para adicionar novos recursos potenciais e posteriormente reservas de hidrocarbonetos numa área localizada perto de grandes cidades da costa do Peru.

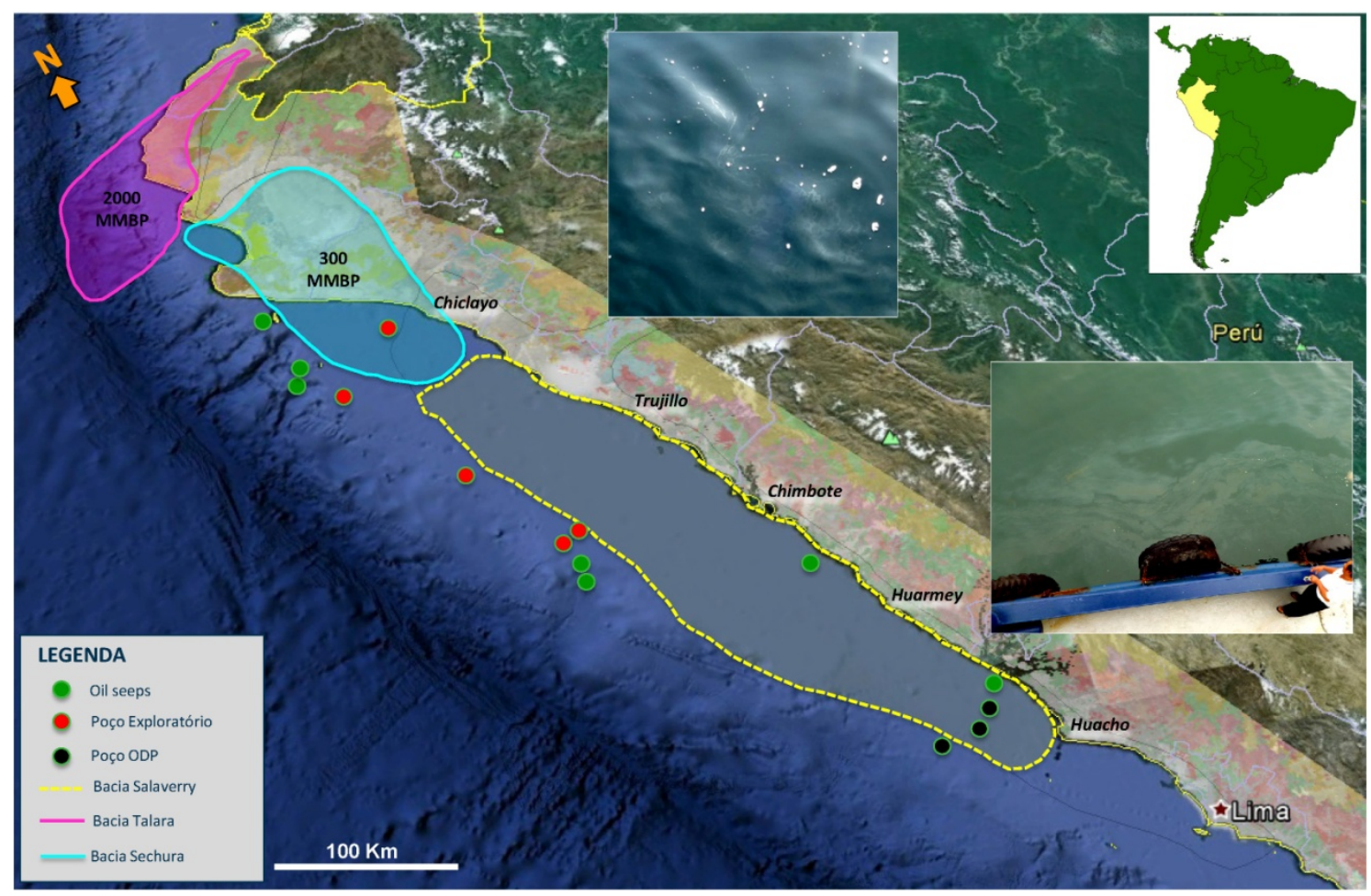

Figura 1. Mapa de localização da Bacia Salaverry (delineada por tracejado amarelo) no segmento de bacias de "fore-arc" (defronte ao arco) prolíferas em hidrocarbonetos, no Peru. Observa-se também a indicação de volume em milhões de barris de petróleo (MMBP) nas bacias Sechura e Talara, poços e oil seeps (modificado de Timoteo et al., 2014).

\subsection{JUSTIFICATIVA}

O presente trabalho justifica-se pelo o potencial petrolífero da Bacia de Salaverry que tem uma base de dados sísmicos $2 \mathrm{D}$ e $3 \mathrm{D}$ pouco explorada e dois poços exploratórios no limite entre as bacias Trujillo e Salaverry (poços Delfin-1X e Ballena-1X) (Figura 2).

Em estudos prévios (Valencia et al., 2011 e Timoteo et al., 2014), no poço Delfin$1 \mathrm{X}$ evidenciou-se gas shows nos intervalos silicilásticos cenozóicos enquanto no poço 


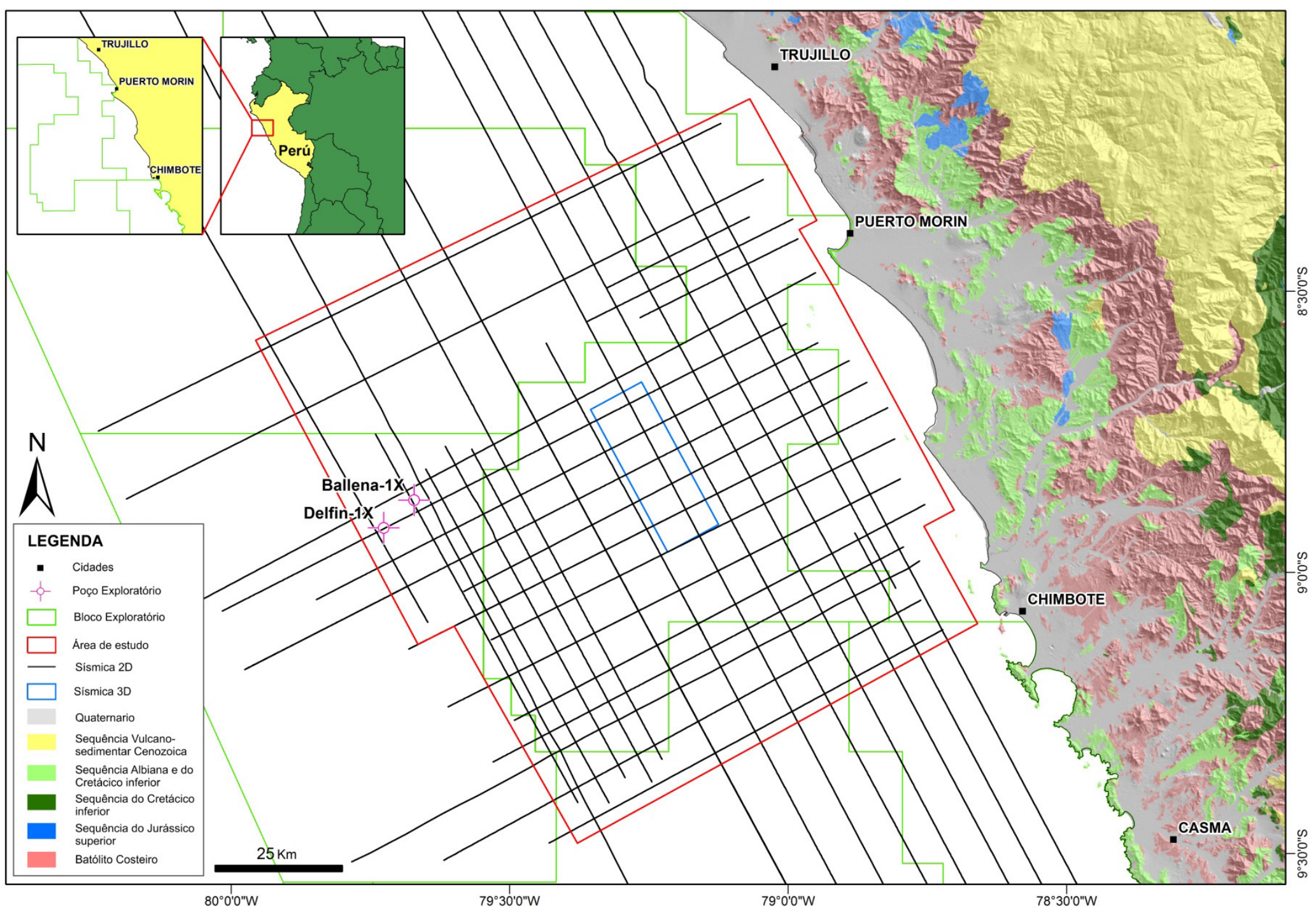

Figura 2. Mapa de localização da área de estudo na porção centro-norte da Bacia Salaverry com a disposição dos dados sísmicos 2D e 3D utilizados no projeto, na seção offshore, assim como as principais unidades estratigráficas aflorantes na margem continental emersa adjacente (onshore). 
Ballena-1X não há, até o momento, ocorrência de hidrocarbonetos. Ainda pode-se definir um local de geração e expulsão de hidrocarbonetos potencial (denominado de Chan Chan oil kitchen), que corresponde a um depocentro Mesozóico reconhecido e interpretado nos dados sísmicos 2D (auxiliado por modelagem térmica 3D. Por meio da modelagem, pode-se inferir que as rochas de idade Jurássica (Tithoniano) ingressaram na janela de petróleo e posteriormente na janela de gás, enquanto as rochas de idade Cretácica (Albiano) atingiram somente a janela inicial de geração de petróleo.

Assim, neste trabalho são integrados novos dados sísmicos 2D e $3 \mathrm{D}$ de boa qualidade com dados de poço, testemunho e afloramento, que permitiram caracterizar com melhor propriedade o arcabouço estratigráfico-estrutural da bacia com a base na interpretação de sismofácies e principais sequências sísmicas, interpretação de sistemas deposicionais e identificação de potenciais rochas reservatório e rochas selantes na sequência cenozóica da Bacia Salaverry. Os estudos sismoestratigráficos foram complementados com análise de traços de fissão em apatita, realizados em rochas siliciclásticas perfuradas no poço exploratório Ballena-1X. Adicionalmente, o presente trabalho permitiu definir melhor as armadilhas já identificadas e novas armadilhas, minimizando os riscos geológicos.

Estudos desta natureza servem como incremento na avaliação de novos recursos prospectivos, auxiliando também na descoberta de novos campos de hidrocarbonetos para a região. Portanto a relevância desta pesquisa é alta e também tem o desafio de ser um dos primeiros estudos deste tipo na Bacia de Salaverry.

\subsection{OBJETIVOS}

- Os objetivos principais desta pesquisa são: (1) a interpretação do arcabouço estratigráfico da Bacia Salaverry utilizando dados sísmicos 2D e 3D; (2) definição de sequências sísmicas; e (3) identificação de sistemas deposicionais.

- Os objetivos específicos da pesquisa incluem:

- Interpretação de linhas sísmicas e identificação de falhamentos e superfícies sismoestratigráficas limítrofes na área de estudo.

- Caracterização de sismofácies e do padrão geométrico das sequências deposicionais. 
○ Interpretação da evolução tectono-sedimentar da região.

○ Identificação de potenciais rochas reservatórios e rochas selantes.

- Análise de potenciais armadilhas (trapas).

A presente dissertação compõe-se de uma parte inicial, na qual são apresentados os capítulos de Introdução, Contexto Geológico e Materiais e Métodos para situar o leitor no tema escolhido. A dissertação é na sequencia complementada com o manuscrito intitulado "Tectono-sedimentary evolution of the Salaverry Basin, Peruvian Andes Forearc: New insights in assessing potential reservoirs and plays", submetido ao Journal Marine and Petroleum Geology.

\subsection{MATERIAIS E MÉTODOS}

Nessa dissertação foram utilizadas 38 linhas sísmicas 2D, um cubo sísmico 3D de $364 \mathrm{~km} 2$, ambos em tempo, e dois poços exploratórios (Ballena-1X e Delfin-1X) com perfis de raio gama, potencial espontâneo, resistividade, densidade e sônico. Esses dados foram fornecidos pela empresa SAVIA PERU S.A. Além disso, amostras de calha e testemunho dos poços exploratórios Ballena-1X e Delfin-1X, foram fornecidas pela Agencia Nacional do Petróleo do Peru (PERUPETRO). Um total de 8 amostras de calha foi coletado, sete pertencentes ao poço Ballena-1X e uma pertencente ao poço Delfin-1X, para datação através de traços de fissão em apatita. Entanto que os testemunhos de sondagem do poço Ballena-1X foram liberados para sua descrição nas instalações de PERUPETRO.

\section{MÉTODO DE TRABALHO}

\section{A. Revisão bibliográfica}

- Levantamento bibliográfico (livros, dissertações, teses e artigos) sobre o contexto geológico regional e sobre a evolução paleogeográfica de área do estudo.

- Leitura aprofundada dos métodos geofísicos utilizados, bem como da utilização de software, se é necessário. 


\section{B. Levantamento de campo}

- Trabalho de campo sobre afloramentos remanescentes de idade Cenozóica localizados ao longo da costa, adjacentes à área de estudo.

\section{Análises das amostras}

- Análises por traços de fissão, nas amostras de calha coletadas.

\section{Análises e interpretação de dados sísmicos}

Análise estrutural

Mapeamento 2D e 3D das principais falhas que controlam o arcabouço estrutural.

Analise de dados dos poços Ballena 1-X e Delfin 1-X (Vail et. al., 1991)

$>$ Integrar rocha e perfil para o reconhecimento das superfícies estratigráficas e sequências deposicionais.

Construir o sismograma sintético do poço utilizando os perfis de densidade e sônico.

Realizar a amarração dos poços com os dados sísmicos.

Avaliar as respostas das unidades sísmicas nos poços.

Análise de sequência sísmica (Vail et. al., 1987, Catuneanu et. al., 2011)

Marcar as superfícies estratigráficas pelo reconhecimento sistemático dos padrões de terminação das reflexões.

Estender ou extrapolar os limites de sequências sobre toda as seções sísmicas 2D.

> Correlacionar as sequências no cubo sísmico 3D e gerar um arcabouço tridimensional.

Mapeamento dos tratos de sistema dentro das sequências diferenciadas.

Análise de sismofácies (Mitchum et. al., 1977, Shell Oil Company, 1987)

Reconhecer e delinear as fácies sísmicas dentro de cada sequência através dos parâmetros das reflexões: configuração interna, continuidade, amplitude, frequência e a forma externa dos pacotes de reflexões. 
Mapeamento da geometria das fácies sísmicas reconhecidas.

$>$ Associação areal das fácies sísmicas e interpretação de sistemas deposicionais.

\section{Interpretação integrada}

Construir mapas de contorno estrutural dos limites de sequências.

- Elaborar mapas de isópacas de cada sequência.

$>$ Construir mapas de fácies sísmicas de cada sequência.

$>$ Integrar a análise sismoestratigráfica com os dados de traços de fissão e interpretar a evolução tectono-sedimentar da área de estudo.

Construir a carta cronoestratigráfica regional.

$>$ Interpretar a distribuição de rochas reservatório potenciais na área de estudo.

\section{TERMOCRONOLOGIA EM APATITA}

As análises de termocronologia de "traços de fissão em apatita" (TFA) foram realizadas na Universidade Federal do Rio Grande do Sul. Os grãos de apatita foram concentrados pelas técnicas convencionais de separação hidrodinâmica, magnética, líquidos densos e separação a mão uma lupa binocular; para posteriormente ser montados numa resina epóxi. Depois os minerais foram lixados e polidos. As montagens de apatita foram cobertas com laminas de mica a fim de obter as idades dos traços de fissão usando o método do detector externo (Gleadow, 1981).

A irradiação de Nêutron foi realizada no FRM-II reator em Garching (TU München, Germany). Os detectores de mica foram revelados por gravura em HF 48\% por 18 minutos. A contagem dos traços de fissão foi realizada em um microscópio Leica sob ampliação de 1000x. As idades dos traços de fissão foram calculadas usando a zeta calibration approach (Hurford e Green, 1982, 1983) com o dosímetro CN5 e idade padrão da apatita de Durango. As idades são expressas como idades centrais com variação percentual (Galbrait e Lasllet, 1993). Para cada grão de apatita analisado, um valor meio para o parâmetro cinético Dpar foi determinado a partir de cinco medições (Donelick et al., 2005).

Com base na distribuição das idades dos traços de fissão de cada grão de apatita, os comprimentos dos traços confinados, os indicadores cinéticos como Dpar, e 
restrições geológicas independentes; a trajetória Temperatura-tempo $(\mathrm{T}-\mathrm{t})$ para cada amostra foi modelada usando o programa HeFTy (Ketcham, 2005) e o algoritmo multikinetic annealing de Ketcham et al., 2007. As restrições para o modelado por inversão utilizado foram: (a) uma restrição inicial foi definida a $200 \pm 20^{\circ} \mathrm{C}$ em um intervalo de tempo mais velho que a correspondente idade aparente da apatita; (b) uma restrição foi definida em um grande intervalo de temperatura $\left(\sim 80-20^{\circ} \mathrm{C}\right)$ no tempo da idade do grão de TFA mais antigo; (c) uma grande caixa de $\mathrm{T}-\mathrm{t}$ foi aplicada com limites fechados de $\mathrm{T}$ nos limites de temperatura da PAZ (partial anneling zone) dos TFA num tempo correspondente à idade média aparente; e (d) a temperatura atual fornece a restrição final para o modelado. 


\section{CAPÍTULO 2: CONTEXTO GEOLÓGICO}

\subsection{MECANISMOS QUE CONTROLAM A REGIÃO DEFRONTE AO ARCO}

A região defronte ao arco (fore-arc) ocorre em margem ativa de subdução de litosfera oceânica e litosfera oceânica ou continental entre o arco vulcânico de ilhas ou magmático continental e a zona de subducção (fossa ou trincheira).

Os modelos contrastantes das margens convergentes ativas mostram que algumas zonas de subducção são dominadas por um enorme prisma de acresção de abundantes sedimentos e rochas oceânicas, enquanto outros, mais especificamente em arcos magmáticos continentais, mostram erosão tectônica onde o fore-arc compõe-se de rochas continentais que são progressivamente deformadas e subduccionadas, resultando em subsidência e extensão (Hussong 1980; von Huene and Scholl, 1991 em Ramos 2010).
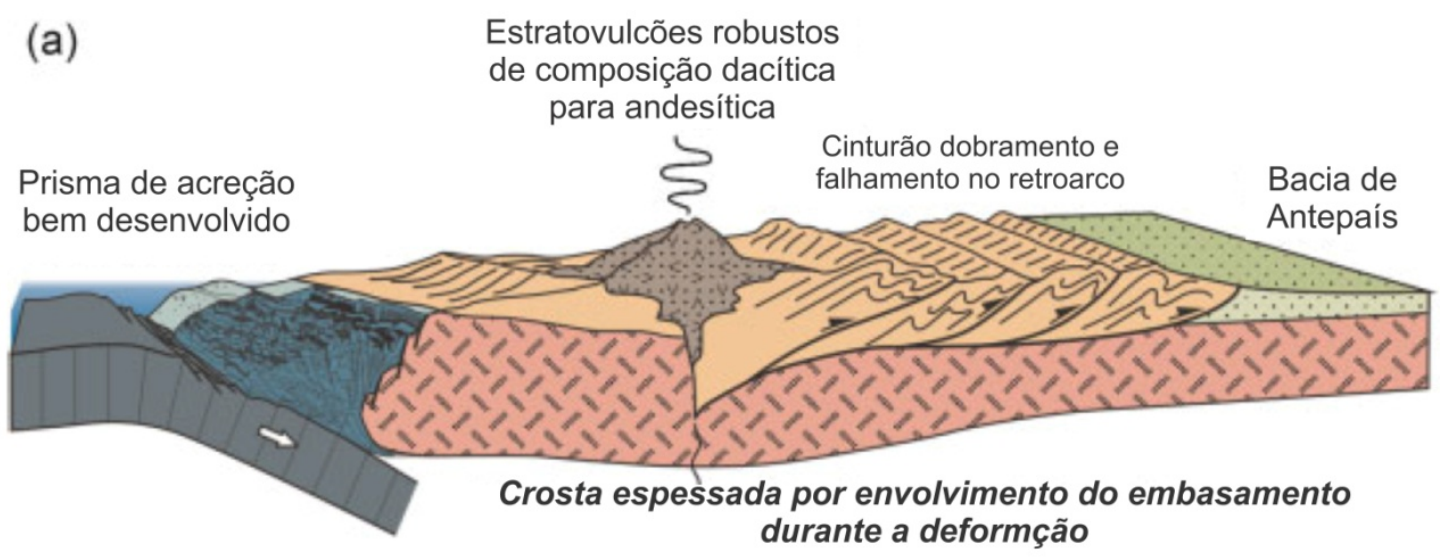

(b)

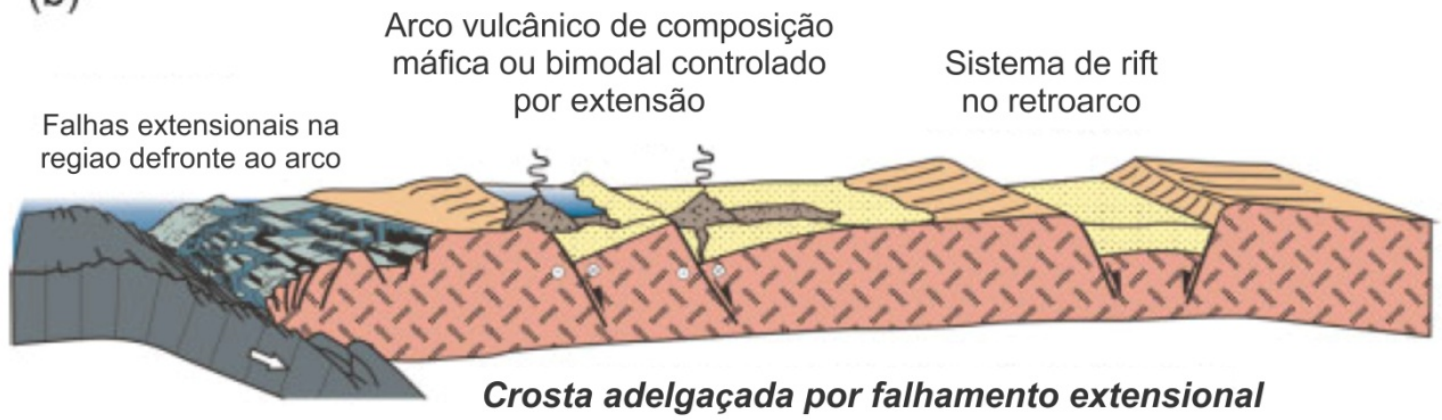

Figura 3. Modelos conceituais nas margens de tipo Andino: (a) margem compressiva, que é a configuração dominante na maior parte dos Andes hoje em dia; (b) margem trativa como vista na parte norte de America Central (segundo Ramos, 2010). 
Ao longo dos Andes há bons exemplos de diferentes tipos de subducção, onde se podem ilustrar os dois modelos conceituais principais (Figura 3). Assim, trincheiras do tipo Andino são, na verdade, frequentemente caracterizadas pela erosão crustal por subducção ao invés de acreção. Portanto, estes modelos devem ser tomados como pontos extremos de configurações possíveis, na medida em que pode ocorrer a combinação de erosão por subducção na região defronte ao arco (contexto extensional no fore-arc, Figura 3b), com um retroarco controlado por compressão convencional (Figura 3a) (Ramos 2010).

Durante os anos 80, basicamente os ciclos de deformação nos Andes foram explicados pela variação da velocidade de convergência. De acordo com isso, se for colocada a velocidade de convergência versus o tempo geológico, pode-se deduzir que o valor máximo de velocidade da Placa de Nazca para o Cenozóico ocorreu a $20 \mathrm{Ma}$ (Mioceno). Do Mioceno ao presente ocorreu gradativamente, por um lado, a diminuição velocidade. Por outro lado, apesar de que a velocidade de convergência de placa tenha diminuído, houve uma deformação crescente nas montanhas andinas (por exemplo, as fases de deformação "Quechua 1", "Quechua 2" e "Quechua 3" se desenvolveram durante este período; Figura 4). Tal fato, induz que a velocidade de convergência não controla necessariamente a deformação nos Andes (Ramos, 2009a).

A subducção de uma crosta continental por uma placa oceânica está longe de ser uma simples interação de placas tectônicas. Há vários fatores que afetam os processos geológicos e seus produtos finais ao longo de uma margem continental ativa. No entanto, há certo consenso sobre os principais parâmetros que controlam a geometria, o acoplamento e o ambiente tectônico da zona de subducção de tipo Andino.

Os parâmetros de primeira ordem são relacionados a: (i) o movimento absoluto da placa superior relativo ao marco referencial do "ponto quente Pacífico" e a consequente velocidade de retrocesso da trincheira (Vt), e (ii) também o acoplamento de cisalhamento na interface das placas. Todos estes parâmetros de primeira ordem devem ser combinados com as características de segunda ordem. Localmente, as influências do preenchimento da trincheira são associadas ao clima dominante na região defronte ao arco e à idade da crosta oceânica subduccionada, i.e., têm papéis secundários. Do mesmo modo colisões de dorsais oceânicas sísmicas e não sísmicas e colisões de zonas 
de fratura têm também um resultado local que pode produzir um aumento no acoplamento que reforça a deformação compressiva.

A cinemática entre a placa superior e inferior no sistema de subducção Andino pode ser expressa pela relação entre o retrocesso da linha de trincheira e o movimento da placa superior com respeito à linha da trincheira dentro de um ponto de referência na astenosfera. Isso é correto apenas em um modelo de manto passivo, porque se um for postulado um movimento global para o oeste da litosfera, tais premissas não são válidas (Doglioni et al. 2009 em Ramos, 2010).

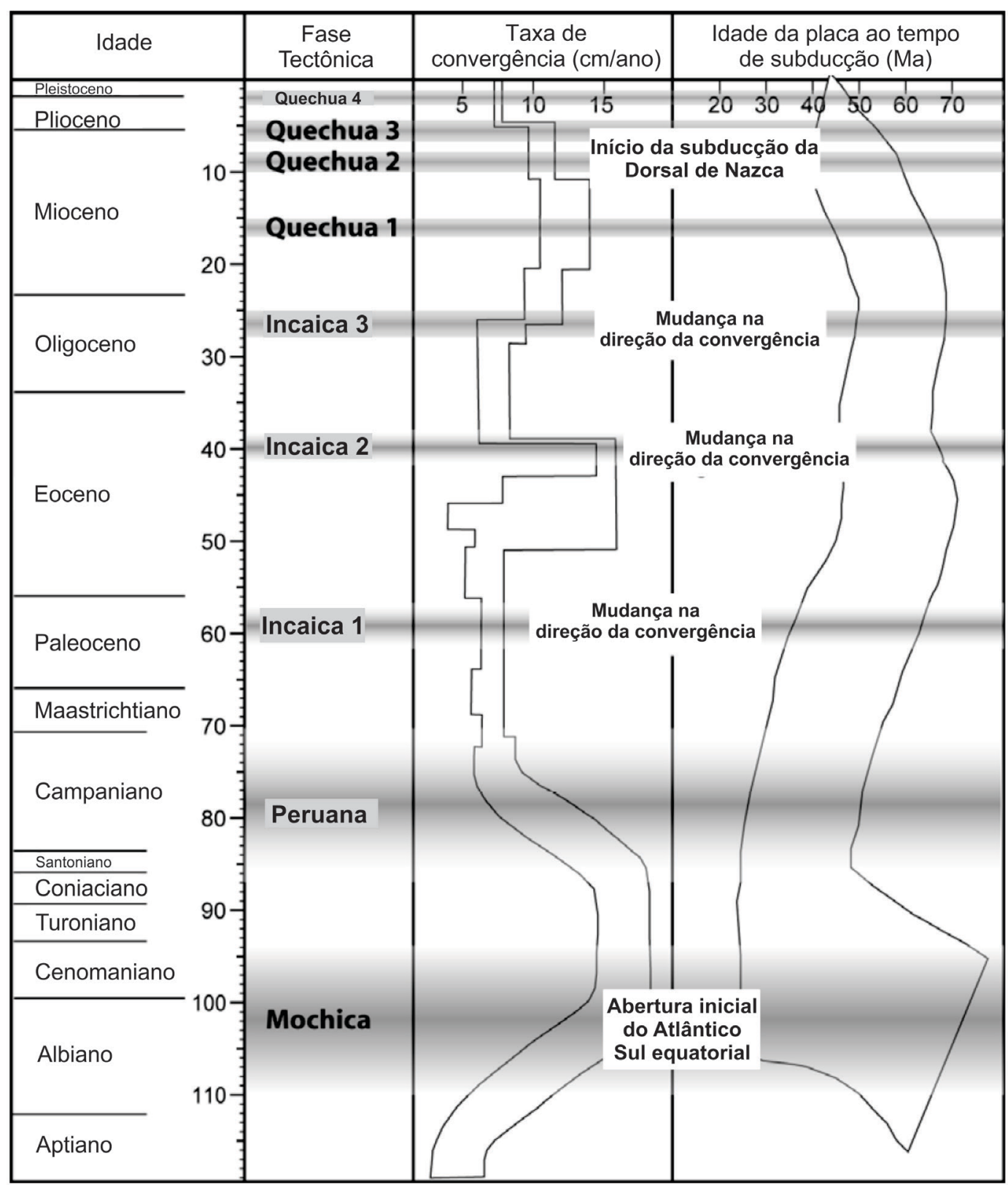

Figura 4. Relação entre os eventos tectônicos, a taxa de convergência (ou velocidade de convergência) e a idade da placa oceânica durante a subducção na margem oeste de America do Sul (modificado de Jaillard et al., 2000 em Wipf, 2006). 
Se forem consideradas a taxa de encurtamento (Vsh) do sistema compressivo e a taxa de estiramento (Vst) de um sistema extensional, a relação entre a taxa de retrocesso da trincheira $(\mathrm{Vt})$ e o movimento absoluto (Vup) pode ser expressa como colocado na Figura 5. O sistema é, obviamente, melhor representado se a erosão crustal (Ver) é tomada em consideração.

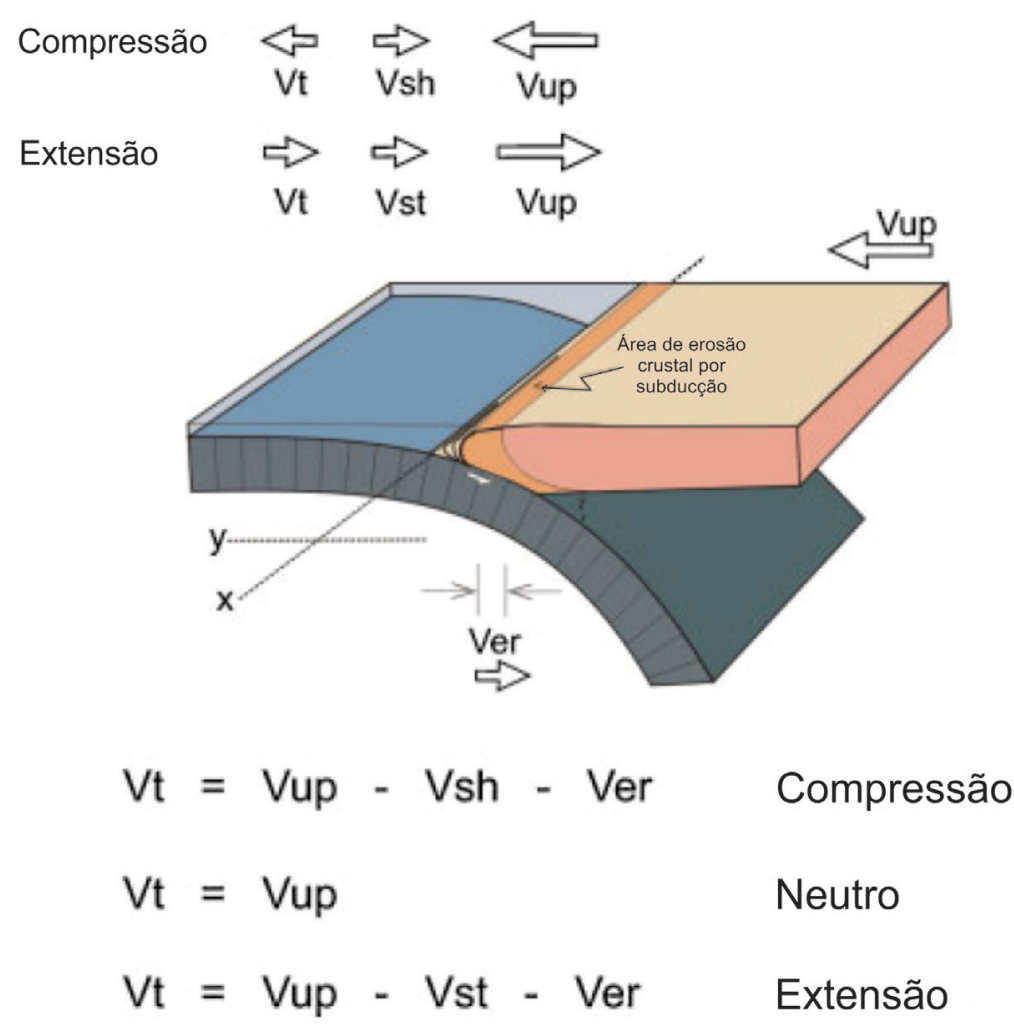

Figura 5. Principais parâmetros cinemáticos que controlam o regime tectônico em uma margem do tipo Andino. Vt: velocidade de retrocesso da trincheira; Vup: movimento absoluto da placa superior; Vsh: taxa de encurtamento; Vst: taxa de alongamento e Ver: taxa de erosão crustal (segundo Ramos, 2010).

Estas relações foram aplicadas à totalidade dos Andes mostrando que o movimento absoluto da placa superior (Vup) relativo ao marco referencial do "ponto quente Pacífico" era um dos parâmetros mais importantes para predizer o esforço tectônico através do tempo, tal como representado pela velocidade de retrocesso da trincheira (Vt). Portanto, um controle de primeira ordem é o movimento absoluto da placa superior (Vup) relativo ao marco referencial do "ponto quente Pacífico", que produz a retirada da linha de trincheira, se afasta da placa superior (retrocesso da trincheira) e gera extensão generalizada (Vt $<0$, Figura 6b); ou, também, produz o avanço da placa superior na direção da placa oceânica gerando um forte acoplamento e uma robusta compressão (Vt $>$ 0, Figura 6c). Alguns exemplos mostram que a linha de charneira 


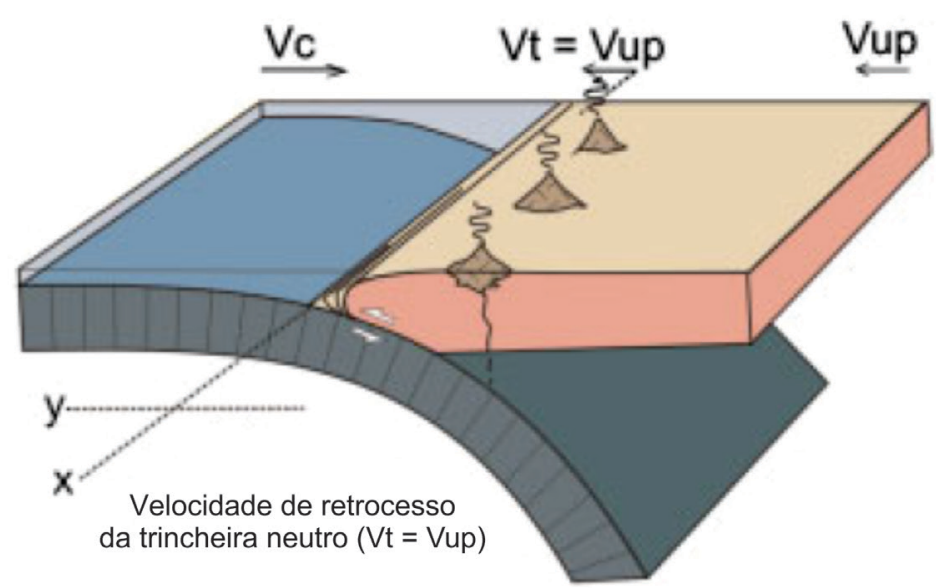

(a) Linha de trincheira estacionária

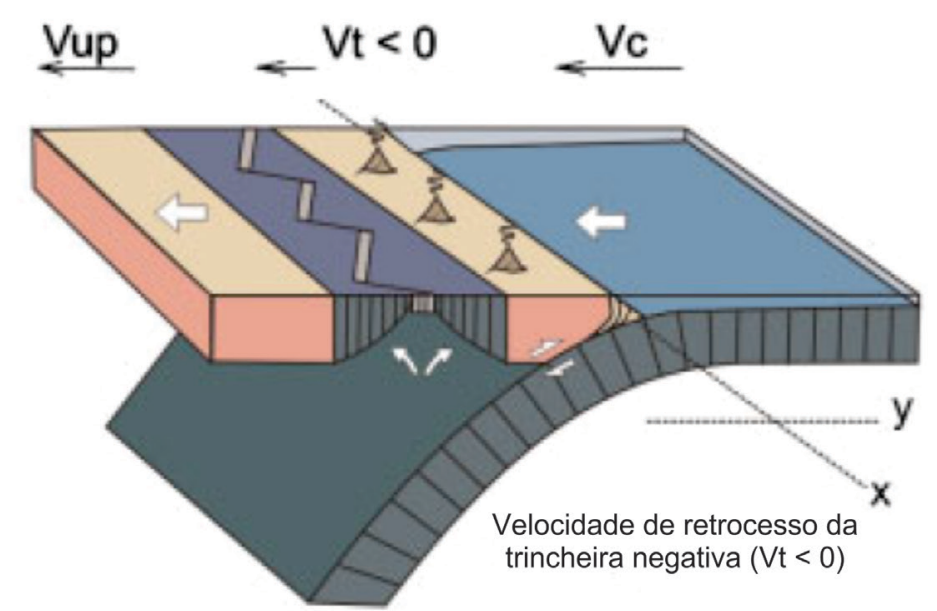

(b) Linha de trincheira em recuo

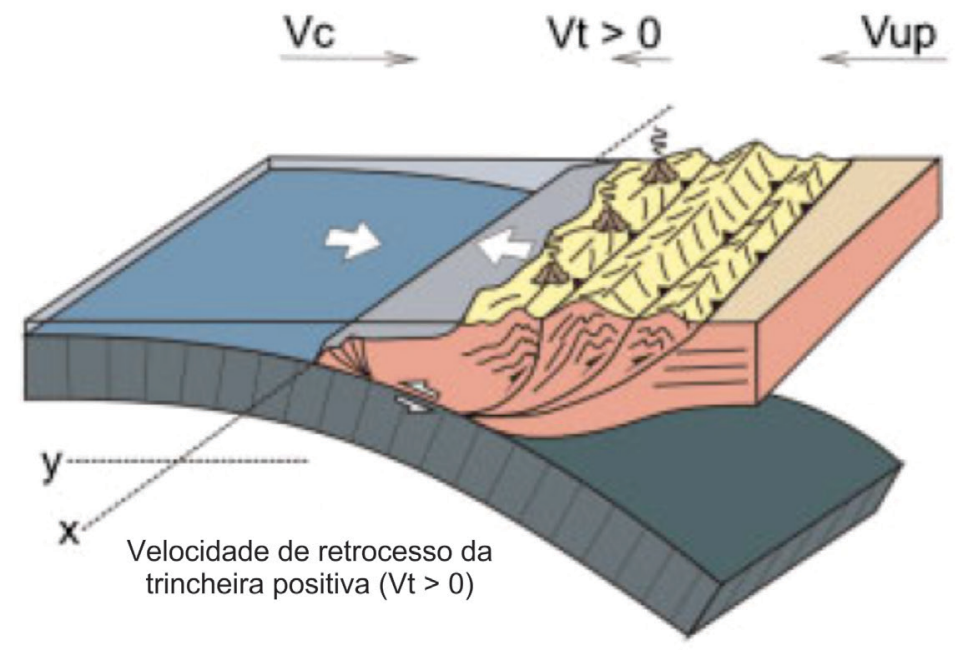

(c) Linha de trincheira com migração frontal

Figura 6. Principais cenários na configuração Andina atual: (a) velocidade de retrocesso da trincheira igual a zero; (b) velocidade de retrocesso da trincheira negativa $(\mathrm{Vt}<0)$ na margem tipo Marianas; (c) velocidade de retrocesso da trincheira positiva $(\mathrm{Vt}>0)$ na margem tipo PeruChile (segundo Ramos, 2010). 
(hinge line) pode ficar quase fixa em um marco referencial na astenosfera (retrocesso da trincheira neutro, Figura 6a), o que gera um sistema de subducção quase estacionário ao longo do tempo.

O acoplamento de placas é um dos principais fatores que controla o levantamento dos Andes, cujo coeficiente de atrito na placa de subducção é um parâmetro de primeira ordem para transmitir deformação na placa superior. $O$ atrito está diretamente relacionado com a quantidade de sedimentos na trincheira: trincheiras famintas em condições áridas extremas, como a trincheira localizada entre Perú e o norte de Chile, terão o acoplamento máximo; enquanto as trincheiras demasiado preenchidas em climas úmidos, como as localizadas nos extremos sul e norte dos Andes, terão mínimo acoplamento.

A colisão de dorsais oceânicas sísmicas e não sísmicas ou a colisão de zonas de fratura podem gerar mudanças no estado de esforço regional da placa superior e também, nos eventos tectônicos localizados no Orógeno Andino. Além disso, zonas de fraqueza na crosta continental, tais como suturas entre diferentes terrenos Paleozóicos ou Mesozóicos e falhas extensionais de uma fase tectônica anterior, também exercerão um controle importante no estilo estrutural e na deformação Andina. Nos últimos anos, a influência do clima tornou-se evidente depois da proposta de Montgomery et al. (2001).

Alguns controles de segunda ordem na cinemática, tal como colisões de dorsais oceânicas ou colisões de zonas de fratura, podem localmente alterar aquelas deformações relacionadas ao controle de primeira ordem, produzindo, por exemplo, alguma compressão localizada adicional em áreas restritas. Um bom exemplo dessa interação é a colisão da dorsal não sísmica de Chile contra a Placa Sul-americana, que produziu um pulso dentro do soerguimento de montanhas do sul para norte nos Andes da Patagônia. Alguns outros fatores de segunda ordem podem mudar o regime de primeira ordem nos Andes como por exemplo a mudança local do mergulho da placa oceânica, geralmente produzida pela colisão de uma dorsal não sísmica. A colisão da Dorsal não sísmica de Nazca no Perú central, com migração de norte para sul, controlou a diminuição do mergulho (shallowing) da placa oceânica e o aumento de acoplamento que levou a uma deformação simultânea, a qual se propagou de norte para sul no antepaís até o final da estruturação do cinturão de dobramento e falhamento sub- 
Andino. Este processo controlou também importantes mudanças no magmatismo com a redução da atividade magmática na região de placa com baixo mergulho (placa de subducção sub-horizontal).

Variações de esforço locais no passado e presente dos Andes não estão relacionadas principalmente com as variações na taxa de convergência relativa (pode ser expressa pela velocidade de convergência), a qual é menos importante do que o movimento absoluto da placa superior relativo ao marco referencial do "ponto quente Pacífico" (pode ser expresso pela velocidade de retrocesso da trincheira; Vt), ou mudanças no estado térmico da placa superior.

\subsection{CICLO OROGÊNICO ANDINO}

É definido como ciclo orogênico, o ciclo de formação de montanhas que explica como uma região, onde todo era plano, de repente começa crescer uma montanha e termina em uma cordilheira. Estes ciclos desenvolvidos na Terra na Europa (Alpes) e na América do Norte (Rochosas) estavam baseados na colisão de placas tectônicas.

No decorrer das últimas décadas pode-se constatar que as montanhas andinas são similares aquelas da Europa e América do Norte. Durante os anos 80 basicamente o arcabouço tectônico do modelo Andino foi fornecido por longos períodos de extensão que foram pontuados por curtos episódios de compressão, dominados pela taxa de convergência ou velocidade de convergência. No entanto, o mencionado modelo Andino integrado com as variações da taxa de convergência desenvolvidas por Pardo Casas \& Molnar (1987) e Somoza (1998) demonstraram que a deformação em alguns momentos se incrementou quando a taxa de convergência diminuiu, como nos últimos 20 milhões de anos (Figura 4). Esses dados também mostraram que a alternância entre compressão e extensão não foi rítmica nem global, nem mesmo ao longo dos Andes, como previamente aceitado. Episódios de deformação foram diácronos (não simultâneos) e as fases diastróficas, quando precisamente datadas, não coincidiam em diferentes segmentos ao longo dos Andes.

Por outro lado, os processos de shallowing (subducção com placas de baixo mergulho ou sub-horizontal) e steepening (subudcção com placas em alto mergulho) da placa oceânica que afetam a margem Andina têm sido reconhecidos em diferentes segmentos, como no norte da Colômbia assim como nos segmentos de subducção sub- 
horizontal (flat-slab) Peruana e Pampeana, no presente. Além disso, trabalhos recentes têm revelado que a atual configuração da subducção sub-horizontal não foi única, ao invés tem sido uma feição comum ao longo do tempo (Ramos \& Folguera, 2009).

A proposta de que esses episódios (shallowing e steepening) não são tão extraordinários têm levado à sugestão que esses processos são os fatores chaves no controle da formação de montanhas e no reconhecimento de um ciclo orogênico, que se assemelha com os processos descritos tempo atrás por Dickinson \& Snyder (1979) no oeste da America do Norte. Com base nessas novas evidencias um ciclo orogênico conceptual é proposto com os seguintes períodos (Ramos, 2009b e Ramos, 2010; Figura 7):

\section{Quietude, ausência de deformação, e magmatismo de arco incipiente}

Períodos de quietude têm sido reconhecidos em diversos momentos em segmentos separados dos Andes. Um dos mais notáveis foi o início de um novo período de subducção ativa depois do rompimento da placa Farallon após 27 Ma, marcando um novo período de magmatismo de arco. Também é reconhecida a subsidência termal, tipo sag, produto da atenuação extensional do período prévio (Figura 7A). Esse período ocorre nos Andes com o início de um novo ciclo de magmatismo posterior à brusca desaceleração do movimento absoluto da placa durante o Oligoceno-Mioceno inferior.

\section{Expansão do arco magmático e deformação associada}

Período de atividade magmática, sem necessidade de variar o angulo de mergulho da placa oceânica, o arco vulcânico começa a expandir-se em direção ao antepaís. Essa migração do magmatismo incrementa o fluxo de calor e posterior desenvolvimento de transições frágil-dúctil na crosta, favorecendo a deformação do embasamento, de tipo pele grossa (thick-skinned tectonics) (Figura 7B). Há também o início do desenvolvimento das bacias de antepaís e os cinturões de dobramento e falhamento. Esse período ocorre nos Andes durante o Mioceno médio a superior.

\section{Shallowing da zona de subducção e espessamento crustal}

Durante o período que há o processo de shallowing com espessamento crustal começa diminuir o ângulo da zona de Benioff e com migração do frente vulcânico. Enfraquecimento térmico associado aumenta o espessamento crustal nessas áreas, 
levando à deformação no antepaís. O desenvolvimento do arco magmático numa crosta espessada induz a colocação de magma na base da crosta, no campo de estabilidade da granada, produzindo um resíduo eclogítico na interface crosta-manto. Inversão tectônica de falhas extensionais formadas em estágios anteriores são comuns, mas a deformação é concentrada na frente (front) orogênico onde a tectônica de pele grossa (thick-skinned) e de pele fina (thin-skinned) estão ativas. Extenso desenvolvimento das bacias de antepaís acompanha a migração da frente de cavalgamento (Figura 7C). Esse período ocorre nos Andes durante o Mioceno superior (Orogenia Quechua).
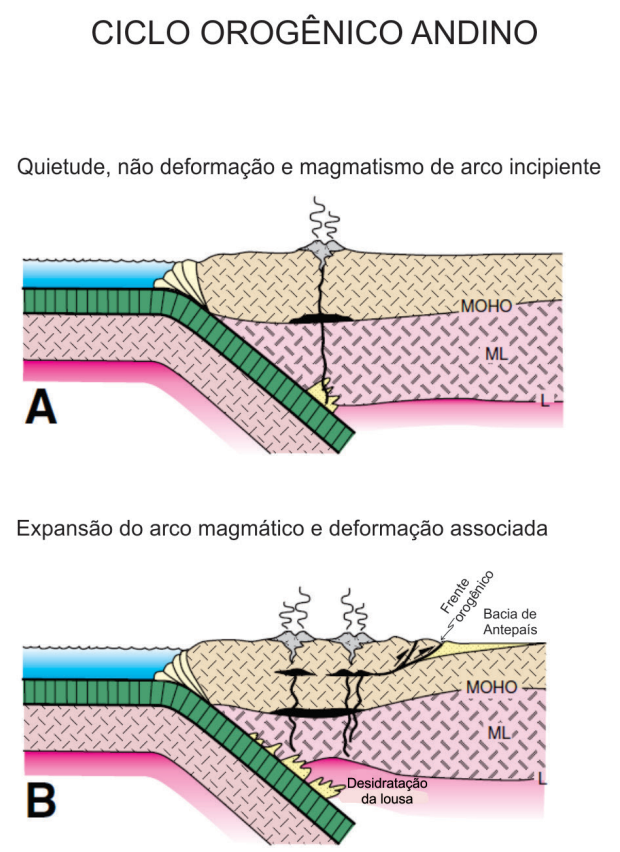

Shallowing da zona de subduç̧ão e deformação crustal

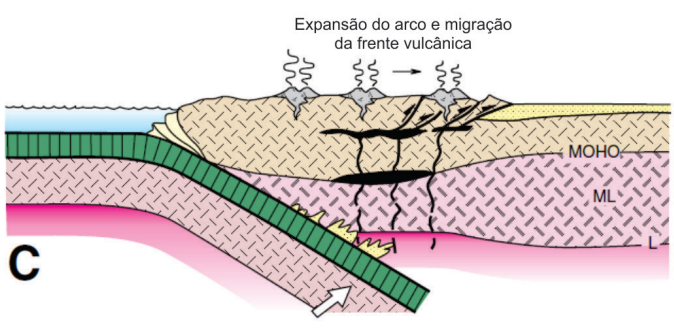

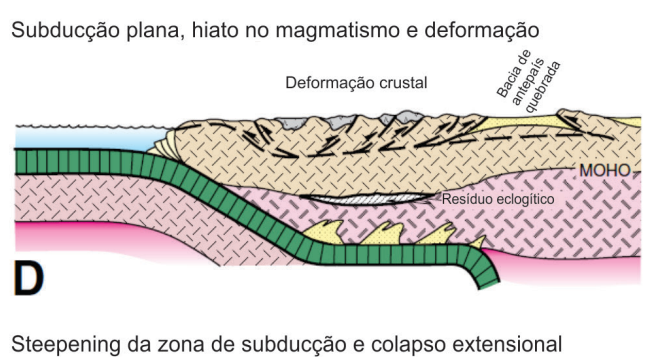

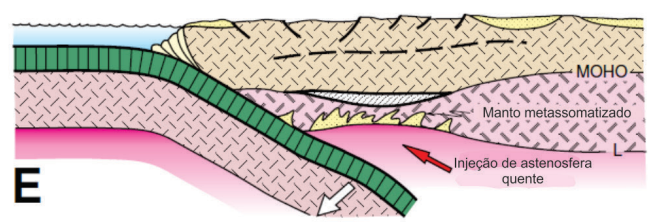

Recuo do arco magmático e delaminação crustal e litosférica

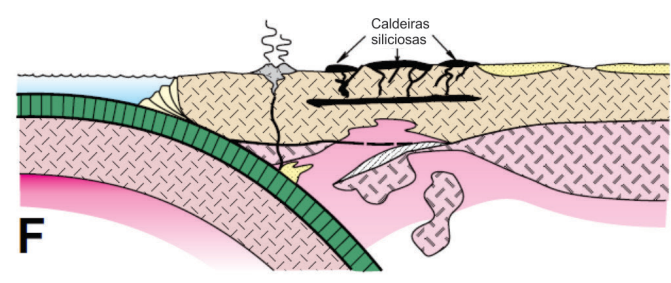

Levantamento e deformação final do antepaís

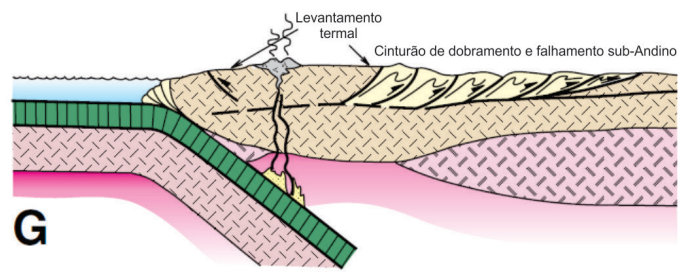

Figura 7. Evolução tectônica idealizada dum Ciclo Orogênico Andino. L: limite litosferaastenosfera; ML: manto litosférico (segundo Ramos, 2009b). 


\section{Subducção sub-horizontal (flat-slab), hiato no magmatismo e deformação}

Devido à subducção sub-horizontal, a desidratação da placa oceânica não contribui para a cunha astenosférica, o que proporciona a última atividade efêmera tão longe quanto $700 \mathrm{~km}$ da trincheira e interrompe o magmatismo com desenvolvimento de resíduos eclogíticos. A deformação crustal está no seu máximo e expande-se em direção ao antepaís; o embasamento é deformado e domina a fragmentação das bacias de antepaís. Bacias intermontanas são frequentes, como aquelas no Perú central e nas Sierras Pampeanas, Argentina (Figura 7D). Esse período ocorre nos Andes ao sul do Perú durante o final do Eoceno, produzindo a deformação no antepaís (modificado de Ramos, 2009a, 2009b).

\section{Steepening da zona de subducção e colapso extensional}

Período de hiato magmático e de inicio do steepening da placa, como consequência a cunha astenosférica avança com injeção de material astenosférico quente sobre o manto litosférico hidratado. Produto dessa injeção começa a subida do limite térmico da litosfera (com afinamento do manto litosférico e inicio dum período de extensão) e se interrompe a deformação no antepaís (Figura 7E). Esse período ocorre nos Andes do sul do Perú durante o inicio do levantamento termal do Altiplano no Oligoceno-Mioceno (modificado de Ramos, 2009a, 2009b).

\section{Recuo do arco magmático e delaminação crustal e litosférica}

O material astenosférico quente delamina o manto devido a que a subida do limite térmico da litosfera põe em contato uma crosta inferior eclogítica mais densa com uma astenosfera menos densa. Essa instabilidade crustal gera extenso desenvolvimento de caldeiras riolíticas e fluxos ignimbríticos na região orogênica. Durante esse período predomina a extensão (Figura 7F). Esse período ocorre nos Andes do sul do Perú com o inicio da delaminação crustal no Altiplano durante o Mioceno superior-Pleistoceno (modificado de Ramos, 2009a, 2009b; Kay \& Coira, 2009).

\section{Levantamento e deformação final do antepaís}

Levantamento termal é evidenciado e estudos no Altiplano Boliviano mostram que a antiga interface (sutura) entre o cráton e o terreno Arequipa é novamente reativada com delaminação crustal. Todos esses processos conduzem a um aumento do fluxo de calor do Altiplano e a Puna, o que favorece o comportamento dúctil da crosta inferior e a 
subsequente extrusão da crosta superior para formar o cinturão de dobramento e falhamento sub-Andino. Além disso, o arco magmático implanta-se novamente e começa, de forma incipiente, um novo ciclo de deformação, magmatismo e subsidência (Figura 7G). Esse período ocorre nos Andes do sul do Perú com o desenvolvimento do sistema sub-Andino na atualidade (modificado de Ramos, 2009a, 2009b).

\section{CICLO OROGÊNICO ANDINO NO PERU}

Se integrarmos a parte norte e sul do Perú teremos os sete (7) períodos do Ciclo Orogênico Andino. Na porção norte do Peru têm sido desenvolvidos os quatro primeiros períodos (Figura 7A-D) e atualmente esse setor do Peru se encontra no período quatro (4), dominado pela subducção sub-horizontal e na fase de máxima compressão e deformação (Figura 7D). Enquanto na porção sul do Peru já se tem desenvolvido os sete períodos, sendo que atualmente esse setor já ingressou na fase de delaminação e apresenta um grande desenvolvimento do cinturão de dobra-falha sub-Andino (Figura 7F). Atualmente, quando se observa a evolução dos Andes, deve ser sempre questionado qual é período do Ciclo Orogênico Andino está sendo trabalhado.

\subsection{GEOLOGIA REGIONAL}

A Bacia Salaverry situa-se na plataforma da margem continental ativa da América do Sul, em frente aos Andes Centrais no Perú, entre as latitudes de $7^{\circ} \mathrm{S}$ e $11^{\circ} \mathrm{S}$. Nessa margem convergente Peruana, as principais feições morfo-estruturais que influenciam a subducção entre a Placa Nazca e a Placa Sul-americana são as Zonas de Fratura de Alvarado, Sarmiento, Mendaña e Nazca e a Dorsal não sísmica de Nazca (Figura 8).

- A Zona de Fratura de Alvarado possui trend $\mathrm{N} 52^{\circ} \mathrm{E}$, estende-se ao longo de 340 $\mathrm{km}$ e intersecta a fossa na latitude $4^{\circ} \mathrm{S}$.

- A Zona de Fratura Sarmiento possui trend N51 ${ }^{\circ}$ E, estende-se ao longo de 345 $\mathrm{km}$ e intersecta a fossa na latitude $5^{\circ} \mathrm{S}$.

- A Zona de Fratura Mendaña possui trend N63 ${ }^{\circ}$ E, estende-se ao longo de 1500 $\mathrm{km}$ e intersecta a fossa na latitude $10^{\circ} \mathrm{S}$.

- A Zona de Fratura de Nazca possui trend $\mathrm{N} 62^{\circ} \mathrm{E}$, estende-se ao longo de 3760 $\mathrm{km}$ e intersecta a fossa na latitude $17.5^{\circ} \mathrm{S}$. 
Por outro lado a Dorsal não sísmica de Nazca está orientada segundo $\mathrm{N} 42^{\circ} \mathrm{E}-\mathrm{S} 42^{\circ} \mathrm{W}$ e intersecta a fossa na latitude $15.5^{\circ} \mathrm{S}$ e o litoral peruano na latitude $14.8^{\circ} \mathrm{S}$. Segundo diversos autores o início da subducção da Dorsal de Nazca sob a Placa Sul-americana ocorreu no Mioceno superior ( 11.2 Ma). Já o deslocamento das placas e a orientação da dorsal induziram o movimento da mesma com propagação na direção $\mathrm{SE}$, desde o norte ao sul do Perú central. A subducção da Dorsal de Nazca, de um lado, influenciou eventos localizados de compressão na região defronte ao arco (fore-arc), gerou a subducção sub-horizontal e consequente hiato do arco magmático; e, por outro lado, adicionou deformação compressiva na porção de antepaís (foreland retro-arc) do Peru Central (Hampel, 2002; Alemán, 2006; Wipf, 2006; Espurt et al., 2007 e Baby et al., 2014).

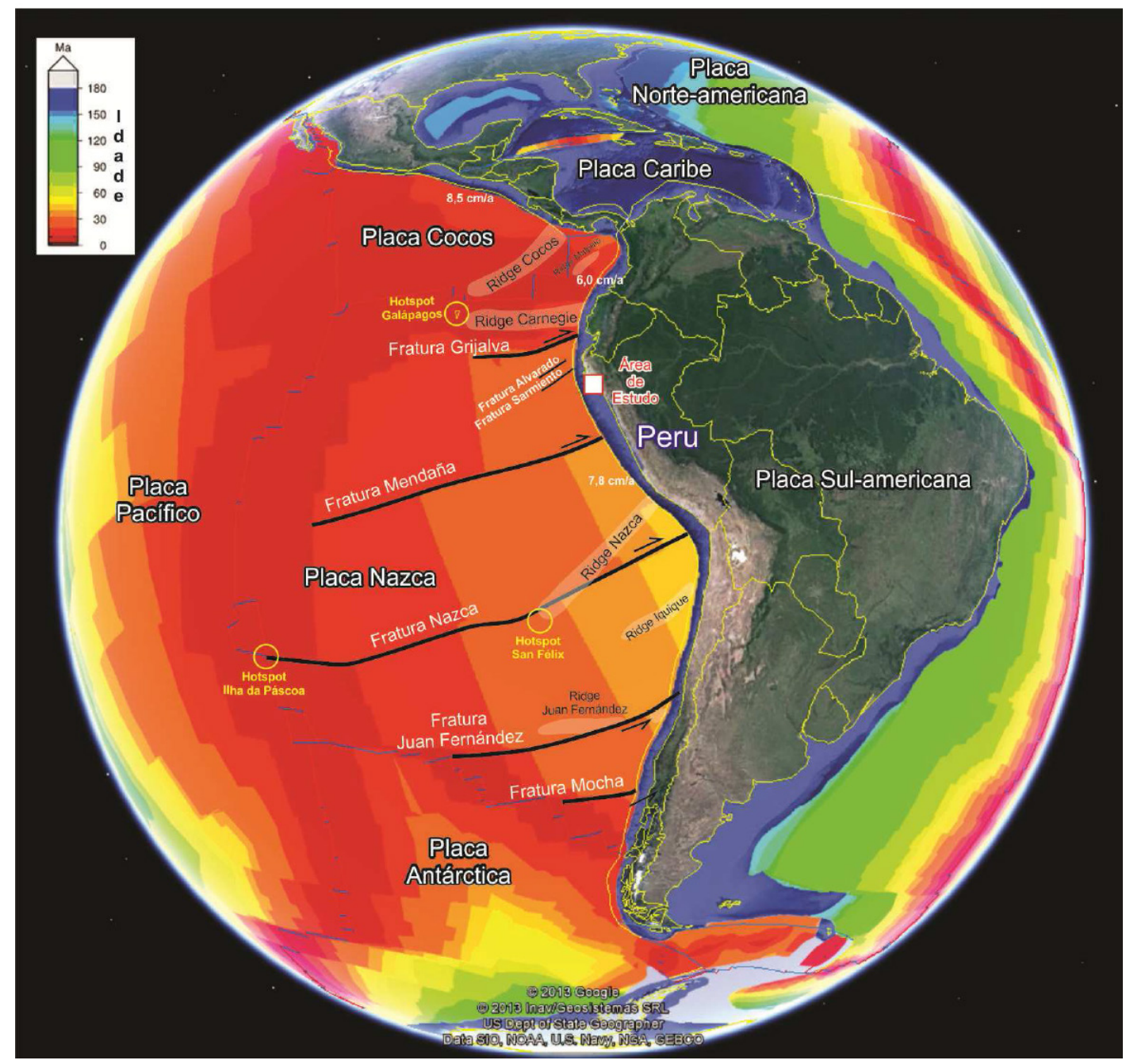

Figura 8. Configuração tectônica da Placa Nazca e sua relação com a área de estudo, a qual está situada na margem oeste da Placa Sul-americana. Estão inseridas as principais feições morfoestruturais, que influenciam a margem continental peruana, e a idade da placa oceânica (modificado de Lajo, 2014). 
Nesse contexto de dinâmica da tectônica de placas, o embasamento da borda ocidental do Perú corresponde ao embasamento siálico composto por diferentes terrenos amalgamados à protomargem ocidental do Gondwana (Figura 9).

O Terreno Paracas, terreno para-autóctono de idade Grenvilliana formado como parte do supercontinente Rodinia, separou-se do cráton Amazônico durante o Neoproterozóico e posteriormente colidiu com o mesmo durante o Ordoviciano. Durante esta colisão formaram-se um arco magmático paleozóico (Ramos, 2009b) e complexo ofiolítico (Willner et al., 2010; Tassinari et al., 2011 e Castroviejo et al., 2014). O Terreno Paracas possui evidencia na plataforma continental de uma cadeia descontinua de altos de embasamento conhecida como "Arco de Paracas" (Aleman \& Ramos, 2000) ou também denominada como “Outer Shelf High" (Kulm et al., 1981). O Arco de Paracas localiza-se na plataforma continental do Peru entre as latitudes de Paracas e Chiclayo, com afloramentos nas Ilhas "Las Hormigas de Afuera", "Lobos de Afuera" e "Lobos de Tierra", e tem sido perfurado em alguns poços exploratórios (Figura 9).

O Terreno Arequipa é um terreno alóctono que colidiu com o cratón Amazônico durante o Mesoproterozóico na formação do supercontinente de Rodinia. A partir daquele momento permaneceu amalgamado na borda ocidental de Gondwana. Ao mesmo tempo, durante essa colisão, o supercontinente Laurentia colidiu com o Terreno de Arequipa, o que originou um cinturão metamórfico de idade Neoproterozóica. Logo após, depois da separação dos supercontinentes Laurentia e Gondwana, o Terreno Arequipa permaneceu conectado ao lado Sul-americano (Ramos, 2008) (Figura 9).

Por outro lado, o Terreno Amotape-Tahuin, localizado no setor norte do Perú e sul de Equador, é proposto como um remanescente do Laurentia que após a colisão com o Terreno Paracas durante a orogenia Alleghanide (no Permiano), ficou amalgamado ao lado Sul-americano. São necessários dados geocronológicos e termocronológicos adicionais que permitam entender a gênese desse terreno e diminuir a especulação nesse modelo (Ramos, 2008; 2009b; Romero et al., 2013 e Ramos, 2014) (Figura 9).

Dessa maneira os três (3) terrenos que conformam o embasamento siálico da borda ocidental do Peru estão limitados por dois (2) grandes alinhamentos que são denominados como a Deflexão de Huancabamba, ao norte, e a Deflexão de Abancay, ao sul (Figura 9). 


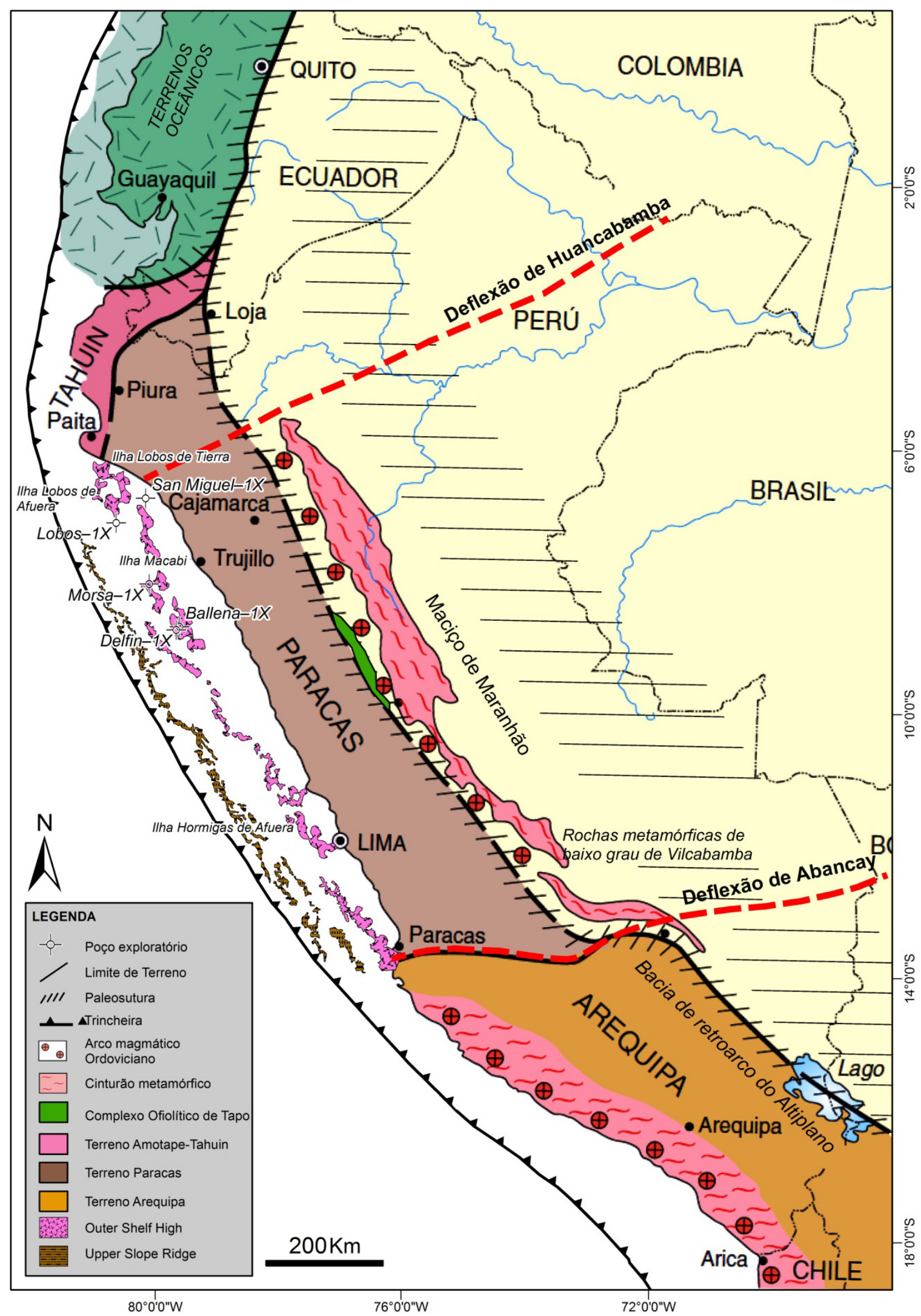

Figura 9. Mapa com distribuição do embasamento nos Andes Peruanos e sua relação com as Deflexões de Huancabamba e Abancay. A sutura oriental (paleosutura) indica a protomargem de Gondwana no Perú (modificado de Ramos, 2014). Na região offshore observa-se o OSH que é evidenciado nas rochas expostas nas ilhas e nas rochas metamórficas perfuradas nos poços exploratórios (modificado de Romero et al., 2013). 
Assim, sobre esse embasamento de idade Pré-cambriana-Paleozóica, desenvolvemse as bacias de fore-arc Peruanas, as quais se encontram limitadas a oeste pelo alto externo da plataforma (Outer Shelf High). Este alto que é interpretado como a extensão offshore da "Cordilheira da Costa" (Figura 10).

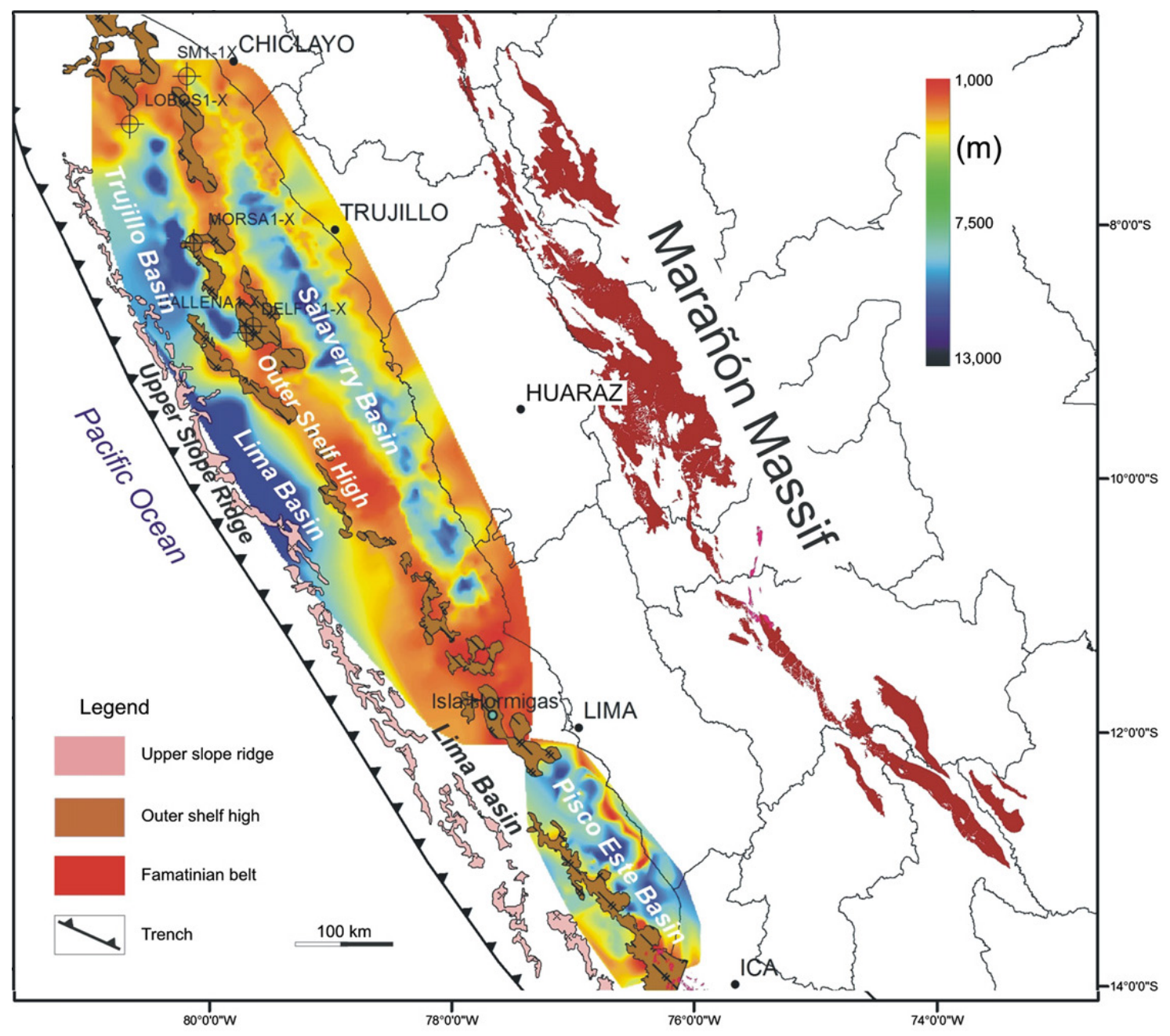

Figura 10. Trend NW-SE das bacias de fore-arc Peruanas, limitada a oeste pelo alto da plataforma externa (Outer Shelf High). O mapa estrutural do topo do embasamento PréCambriano-Paleozóico é baseado em interpretação sísmica, enquanto que as cadeias descontinuas de altos de embasamento são baseadas na interpretação de dados gravimétricos e magnetométricos (Romero et al., 2013).

A Bacia Salaverry faz parte do trend NW-SE das Bacias de fore-arc Peruanas e está localizada imediatamente ao sul das Bacias Sechura e Talara (Figuras 1 e 10). Além disso, a Bacia Cenozóica Salaverry desenvolve-se num ambiente tectônico extensional, com períodos compressivos localizados, e ocorre sobre rochas paleozóicas e jurocretácicas. Estas rochas compõe a bacia paleomesozóica, que evoluiu num contexto 
tectônico extensional associada a um arco vulcânico de margem ativa. Por conseguinte, a coluna estratigráfica da bacia é composta por rochas do Pré-Cambriano, Paleozóico, Mesozóico e Cenozóico.

\subsection{GEOLOGIA DA BACIA}

Ainda quando algumas unidades estratigráficas não afloram em onshore e outras unidades não têm sido perfuradas no offshore, o conhecimento geológico regional baseado na evolução tectono-estratigráfica da margem continental peruana e nos trends regionais das unidades estratigráficas permitem integrar a estratigrafia offshore-onshore da Bacia Salaverry. Nos últimos anos, trabalhos recentes nos afloramentos localizados ao longo da costa e cordilheira ocidental têm permitido corrigir e atualizar o registro estratigráfico da bacia (Valencia et al., 2011, Romero et al., 2013 e Timoteo, 2013, 2014). Tais trabalhos descrevem a estratigrafia da Bacia Salaverry, a qual, na presente pesquisa, é detalhada nas diferentes unidades estratigráficas que compõem o registro sedimentar na área de estudo (Figura 2) bem como serão estudadas segundo os objetivos do projeto e sua importância econômica como elementos potenciais do sistema petrolífero.

\section{EMBASAMENTO PRÉ-CENOZÓICO}

Também denominado como embasamento econômico, é constituído de diferentes unidades sedimentares, vulcano-sedimentares, metamórficas e ígneas que atuam como embasamento dos depósitos Cenozóicos da bacia. O domínio geográfico de cada unidade se diferencia ao longo e largo da bacia.

\section{PRÉ-CAMBRIANO}

Em onshore ainda não se tem evidencias de rochas dessa idade e os afloramentos mais próximos se localizam a partir da latitude de Paracas na Cordilheira da Costa sobre o Terreno Arequipa. Em offshore estaria representado pelas rochas metamórficas que compõem o Arco de Paracas (Outer Shelf High): xistos, quartzitos e gnaisses que afloram nas Ilhas Lobos de Afuera, Lobos de Tierra e Hormigas de Afuera; assim como gnaisses, fillitas, quartzitos e xistos perfurados pelos poços exploratórios Ballena-1X e Delfin-1X (Figura 9). 
A datação mais recente do embasamento metamórfico do Terreno Paracas se realizou num gnaisse de alto grau de metamorfismo amostrado na Ilha Hormigas de Afuera. Ainda a quantidade de zircões é exígua, três importantes episódios de cristalização de zircão são reconhecidos: zircões paleoproterozóicos, mesoproterozóicos (grenvilianos) e ordovicianos (zircões magmáticos de idade Famatiniana) (Romero et al., 2013; Figura 11).

Essas diferentes rochas metamórficas possuem potencial como reservatórios naturalmente fraturados (porosidade secundaria), tendo como caso análogo mais próximo os reservatórios metamórficos fraturados nos Campos de petróleo de "San Pedro" (offshore) e "Portachuelo" (onshore) na Bacia Talara, no noroeste do Peru.
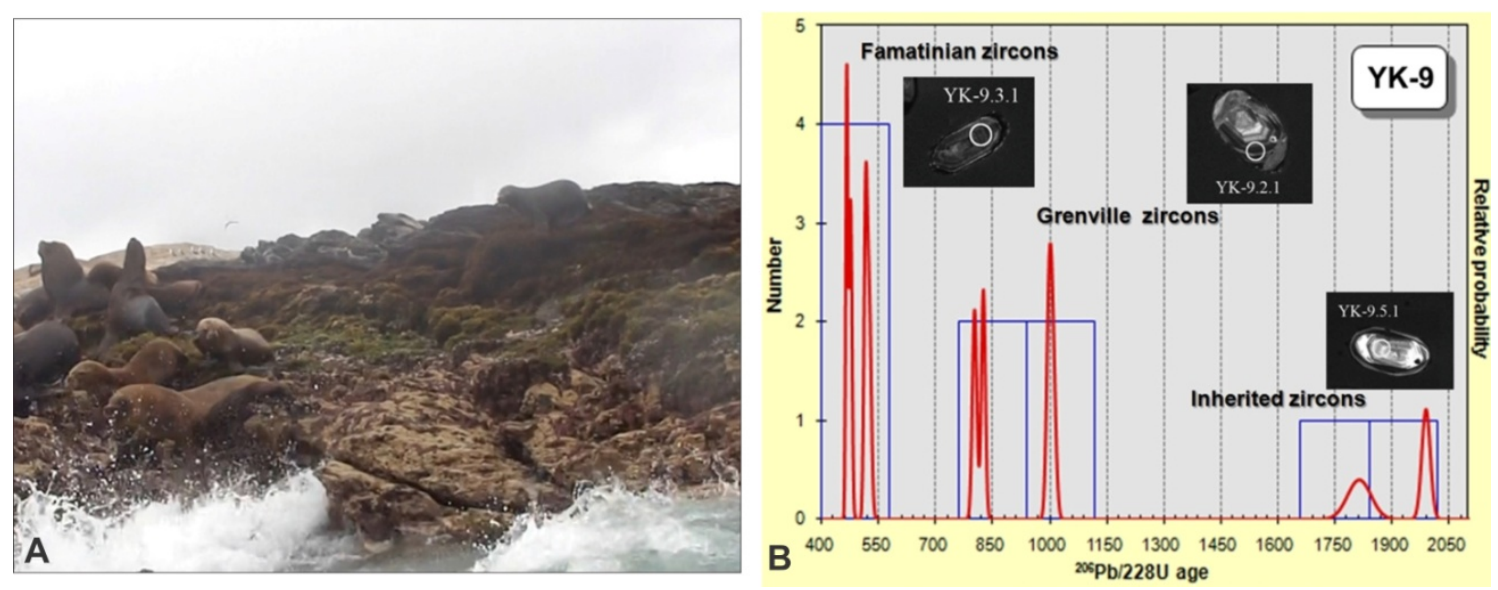

Figura 11. A) Vista da Ilha Las Hormigas de Afuera, onde a amostragem do gnaisse foi realizada. B) Resumo das principais idades dos zircões obtidos no gnaisse: Famatiniano, Grenvilliano, e alguns zircões herdados paleoproterozóicos. Localização da ilha na Figura 9 (Modificado de Romero et al., 2013).
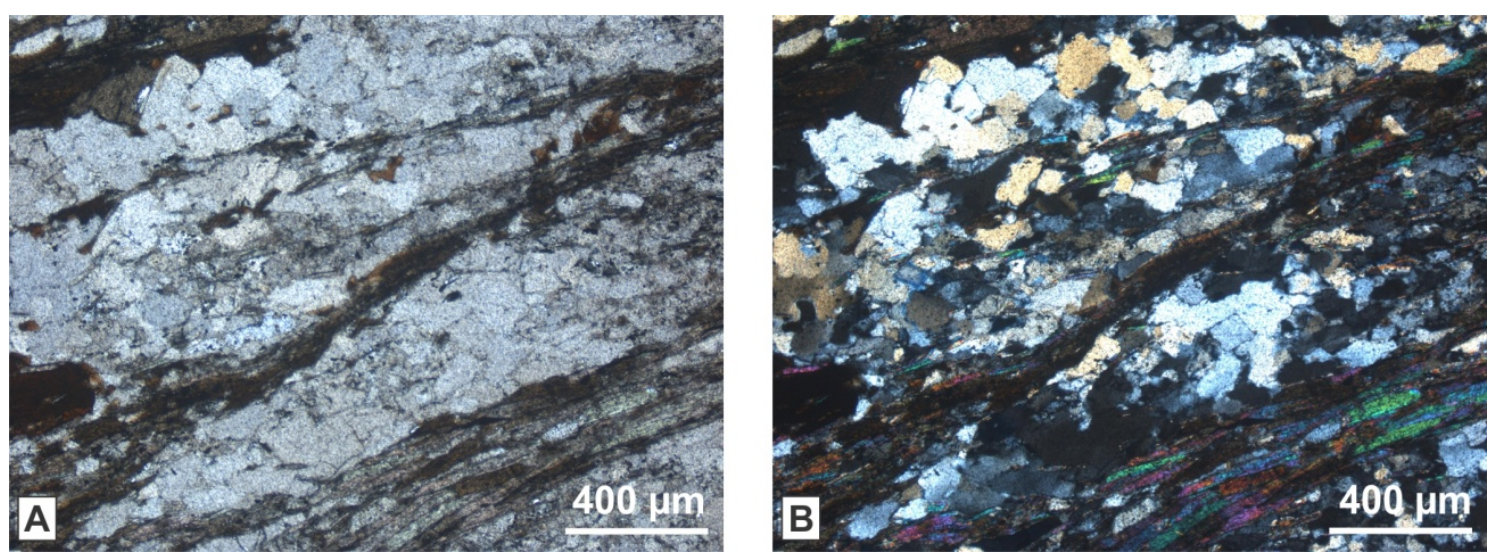

Figura 12. Gnaisse perfurado pelo poço exploratório Ballena-1X: A) microfotografia da lamina delgada em nicóis paralelos e B) microfotografia da lamina delgada em nicóis cruzados. Localização do poço na Figura 9. 


\section{PALEOZÓICO}

Não há rochas paleozóicas na porção aflorante da bacia, mas em offshore estaria representado por rochas ígneas, devido a que alguns grãos de zircão da amostra coletada na Ilha Hormigas de Afuera, que pertence a Bacia Lima, possuem idade Ordoviciana (467,9 \pm 4,5 Ma) (Romero et al., 2013). Além disso, essas idades são similares aos zircões datados no Batólito de San Nicolás ao longo da Cordilheira da Costa sobre o Terreno Arequipa (Figura 9).

Por outro lado, rochas ígneas de composição gabro-diorítica, de idade Permiana $(259.5 \pm 12.3)$, foram perfuradas pelo poço San Miguel-1X, na Bacia Sechura, muito próximo da porção norte da Bacia Salaverry (Timoteo et al., 2012; Ramos, 2014). Estas rochas ígneas correspondem aos remanescentes do arco magmático Permiano e não se descarta que este arco se encontre preservado também em algumas zonas de Bacia Salaverry, ainda que a posição atual do corpo intrusivo com respeito da atual fossa sugere ao menos 200 km de erosão da crosta pós-Permiano.
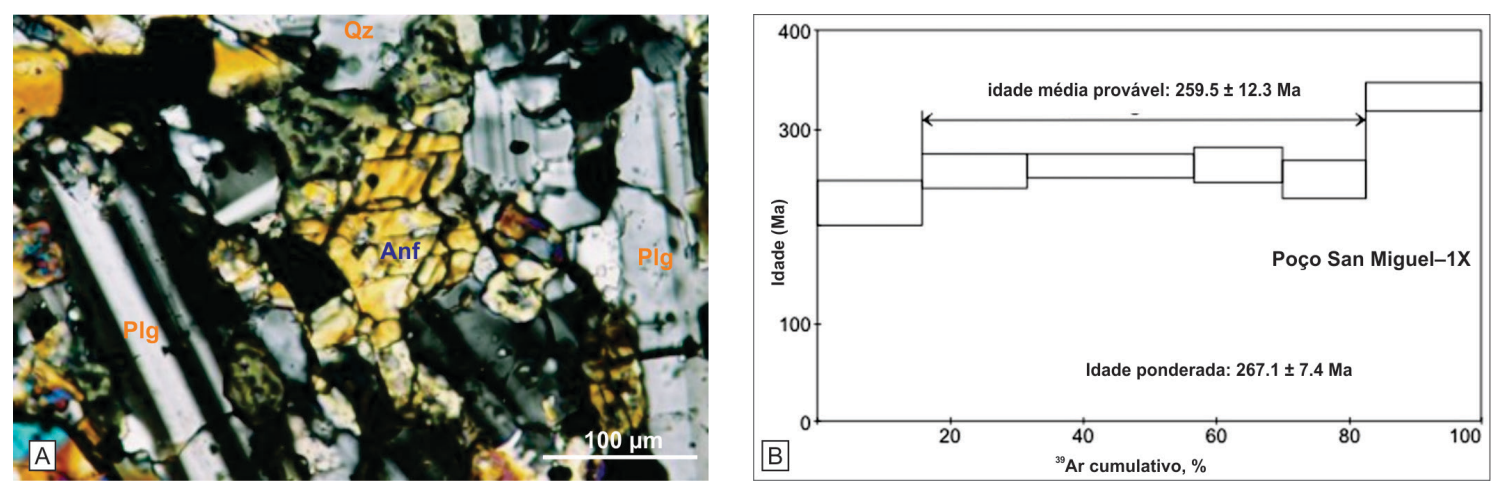

Figura 13. A) Microfotografia da lâmina delgada em nicóis cruzados do diorito perfurado pelo poço exploratório San Miguel-1X. B) Idade Ar-Ar do diorito San Miguel. Localização do poço na Figura 9 (Modificado de Timoteo et al., 2012).

Essas rochas ígneas Paleozóicas possuem potencial como reservatórios naturalmente fraturados, tendo como casos análogos mais próximos os reservatórios ígneos e vulcânicos na região de Rio Grande na Bacia de Neuquén, e no campo Urucu na Bacia de Solimões, respectivamente (Eiras et al., 2003; Zou, 2013). 


\section{MESOZÓICO}

As rochas de idade Mesozóica possuem ampla distribuição ao longo da costa e cordilheira ocidental nos afloramentos adjacentes à bacia.

\section{Triássico - Jurássico}

O registro sedimentar de idade Triásicco-Jurássico é representado por três (3) unidades estratigráficas denominadas como: a Formação La Leche (Triássico superiorJurássico inferior), a Formação Oyotun (Jurássico médio) e a Formação Chicama (Jurássico superior). Na base dessa sucessão estratigráfica há a Formação La Leche, composta predominantemente de calcários e alguns níveis de folhelhos e aflora ao norte da área de estudo no Vale do rio La Leche com espessura entre 200 e 800 m (Figura 14).

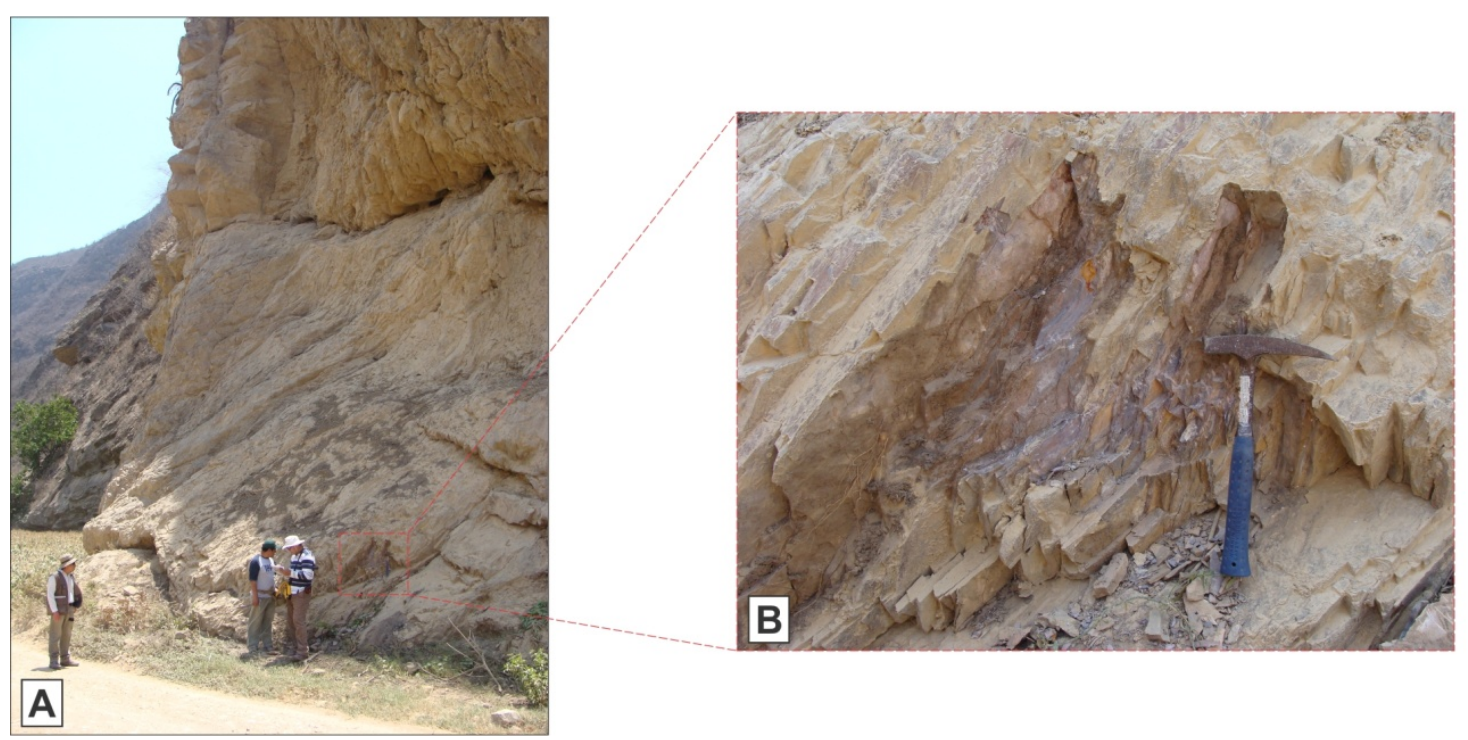

Figura 14. A) Afloramentos da Formação La Leche do Triássico superior-Jurássico inferior, no Vale do Rio La Leche, ao norte da área de estudo. B) Ampliação da foto anterior onde se mostram os calcários cinza escuros na base da secção. Localização do afloramento na Figura 18.

A Formação Oyotun sobrepõe-se a Fm. La Leche e é composta por rochas piroclásticas intercaladas com fluxos lávicos (andesita, basalto) e níveis arenosos. Ocorre entre as localidades de Rio Comaina pelo norte e Cherrepe pelo sul, e possui espessura entre 100 e $500 \mathrm{~m}$.

Para o topo da sucessão ocorre a Formação Chicama que é composta predominantemente por folhelhos pretos a cinzas com intercalações de calcários e níveis 
de arenitos depositados num ambiente marinho profundo. Esta unidade ocorre entre as localidades de Pacasmayo (norte) e Paramonga (sul) com espessura entre 200 e $1200 \mathrm{~m}$ (Figura 15).
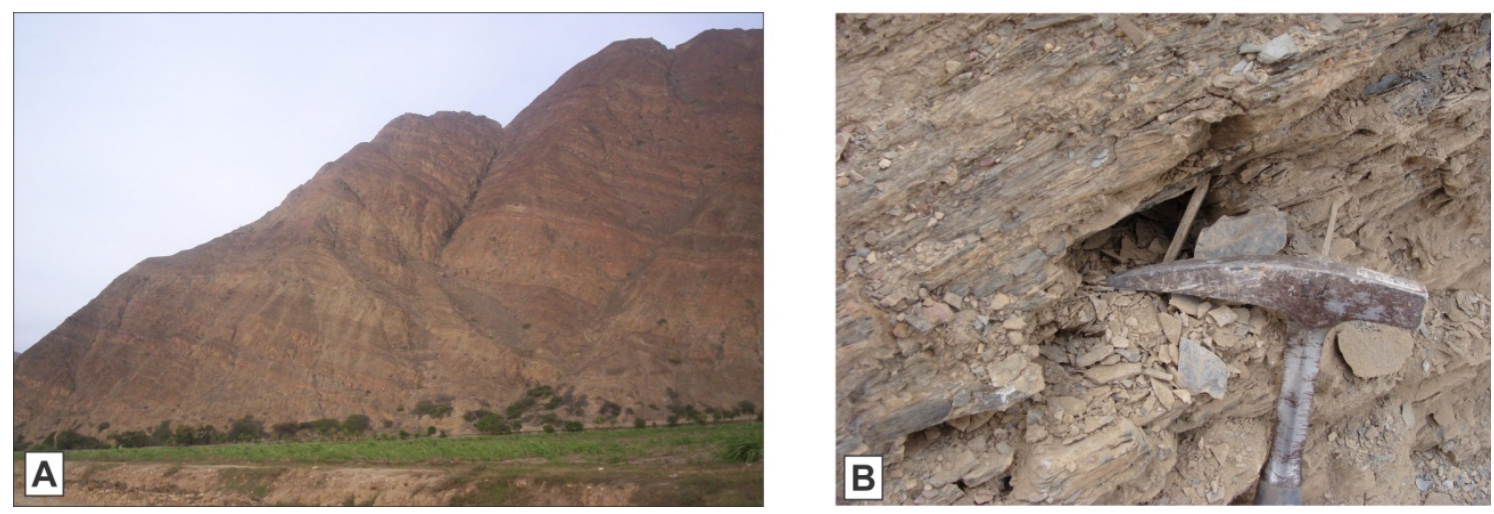

Figura 15. A) Vista panorâmica dos afloramentos da Formação Chicama do Jurássico superior, no Vale do Rio Chicama. B) Folhelhos cinza escuros laminados que conformam os depósitos turbidíticos na seção basal da Formação Chicama (Modificado de Timoteo, 2013). Localização do afloramento na Figura 18.
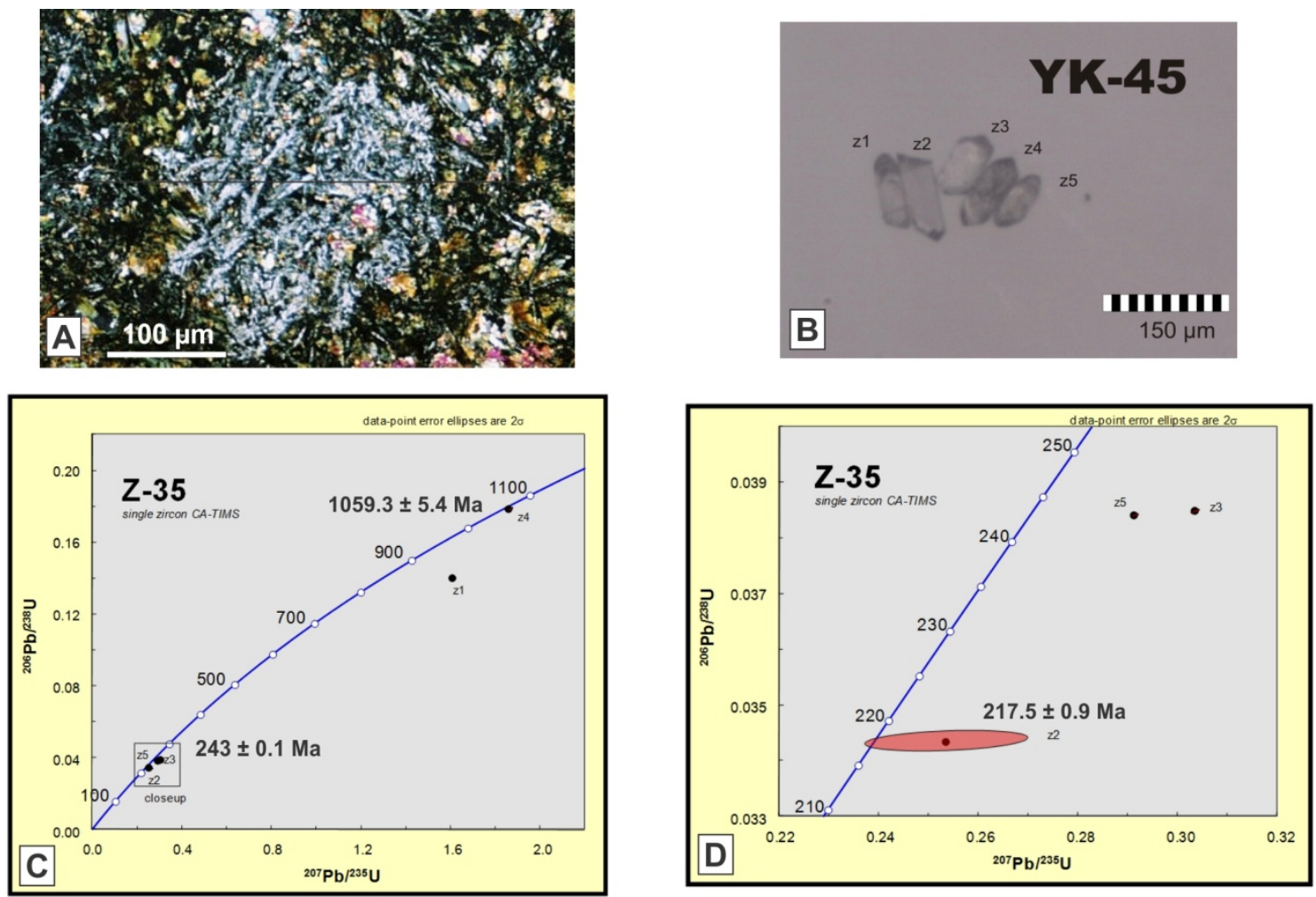

Figura 16. A) Microfotografia da lâmina delgada em nicóis cruzados do basalto amostrado na Ilha Macabi. B) Zircões identificados no basalto e separados para datação U-Pb TIMS. C) Concórdia das idades dos zircões datados no basalto. D) Ampliação da concórdia, onde se observa a idade máxima que poderia atingir o basalto da Ilha Macabi. Localização da ilha na Figura 9 (modificado de Timoteo et al., 2012). 
O basalto da Ilha Macabi, na periferia da Bacia de Salaverry, apresenta zircões herdados datados entre $1059.3 \pm 5.4 \mathrm{Ma}$ e $217.5 \pm 0.9$ Ma. Timóteo et al., 2012 e Ramos, 2014 sugerem que estas rochas básicas possam corresponder à primeira evidencia do arco Jurássico no offshore na Bacia Salaverry (Figura 16). As rochas vulcânicas e os níveis de arenito da Formação Oyotun possuem potencial como reservatórios naturalmente fraturados, entanto que os folhelhos pretos da Formação Chicama são considerados como rocha geradora potencial segundo a caracterização geológica-geoquímica feita nos afloramentos adjacentes à bacia (Valencia et al., 2011).

\section{Cretácico}

As rochas Cretácicas estão compostas por três (3) sequências estratigráficas, denominadas de: Sequência do Cretácico inferior (SCI; Berriasiano-Aptiano), Sequência Albiana (SAL) e Sequência do Cretácico superior (SCS; CenomanianoTuroniano).

Na base dessa sucessão estratigráfica há SCI composta por arenitos quartzosos intercalados com folhelhos e alguns níveis de calcários pertencentes ao Grupo Goyllarisquizga (Figura 17A, B), que ocorrem no norte da área de estudo, entre as cidades de Chicama e Chiclayo, com espessura entre 80 e 2000 m. (Timóteo et al., 2014). A Sequencia Albiana (SAL) é composta na base por lavas, brechas e arenitos vulcanoclásticos da Formação Punta Gramadal que se sotopõem concordantes aos folhelhos carbonosos com intercalações de calcários pertencentes à Fm. La Zorra (Figura 17C, D). Ambas as formações compõem o Grupo Casma. A SAL possui ampla distribuição no Perú central desde Pucusana, ao Sul, até Trujillo, ao Norte, e sua espessura varia entre 100 e 2400 m (Timóteo et al., 2014). Finalmente no topo da sucessão, a SCS sobrepõe-se as unidades anteriores em discordância erosional. Ela é composta predominantemente de calcários com intercalações de folhelhos do Grupo Pulluicana- Quilquiñan, aflorando ao norte da área de estudo entre as localidades de Farrat e Jaen (Timoteo, 2013) (Figura 17E, F).

Os arenitos do Gpo. Goyllarisquizga (SCI) e as rochas vulcânicas do Grupo Casma (SAL) possuem potencial como reservatórios naturalmente fraturados. Por outro lado, os folhelhos carbonosos com alto conteúdo de matéria orgânica da Fm. La Zorra (SAL) são considerados como rocha geradora potencial segundo a caracterização geológica- 
geoquímica realizada nos últimos anos (Valencia et al., 2011; Timóteo et al., 2014). Já calcários da SCS na área de estudo possuem potencial como rochas selantes para os arenitos da SCI e as rochas vulcânicas da SAL, bem como rochas de soterramento para as rochas geradoras potencias depositadas no Jurássico e Cretácico.
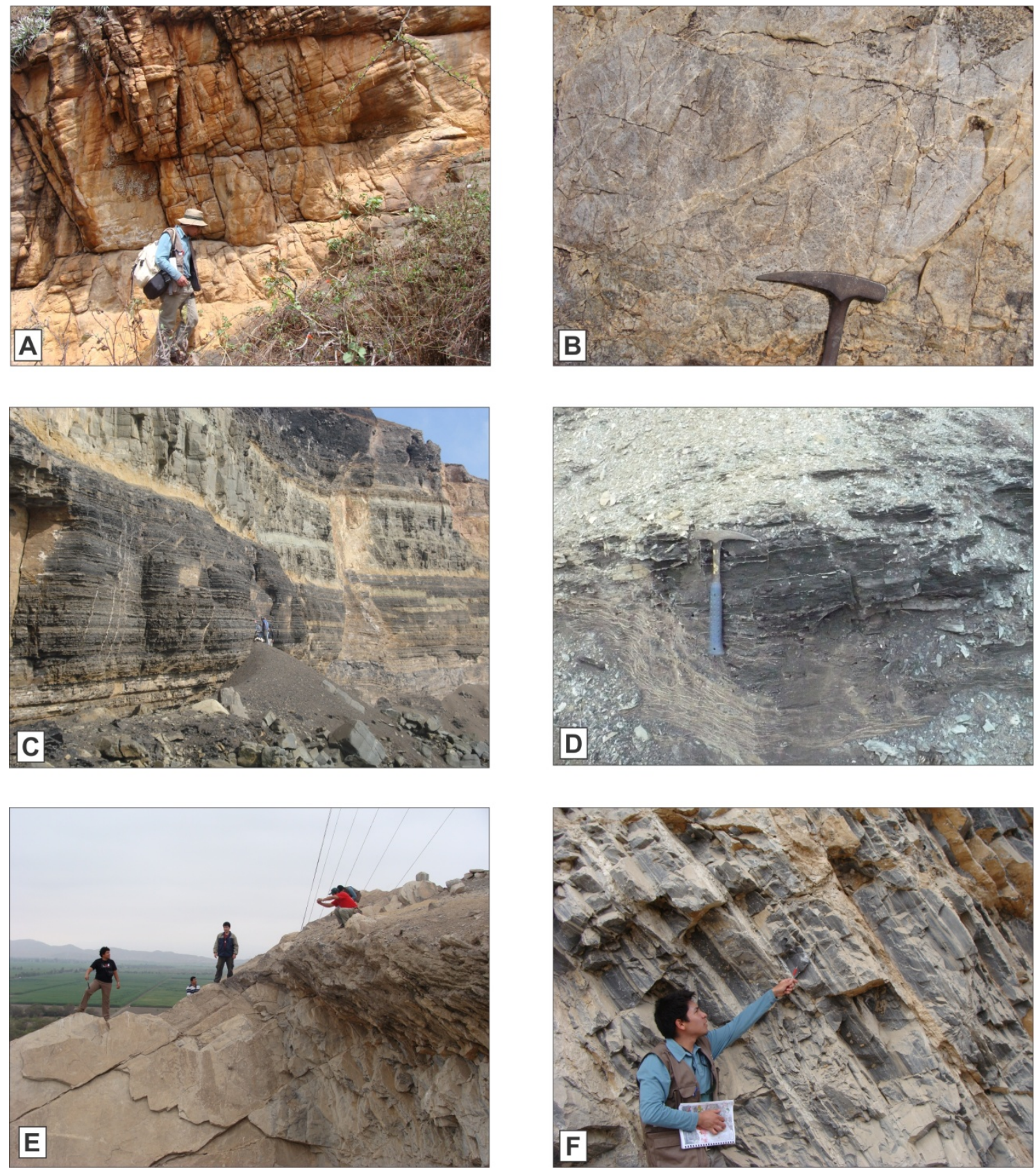

Figura 17. A) Arenitos com estratificação cruzada de grande porte pertencentes ao Grupo Goyllarisquizga na localidade de Simbron. B) Arenitos quartzosos fortemente fraturados pertencentes ao Grupo Goyllarisquizga. C) Afloramentos de folhelhos da Formação La Zorra, Praia La Zorra. D) Folhelhos pretos carbonosos reconhecidos como rocha geradora potencial em toda a secção estratigráfica da Fm. La Zorra. E) Afloramento de calcários do Grupo Pulluicana-Quilquiñan na localidade de Saltur. F) Calcários cinzas escuros fraturados, reconhecidos no topo da secção do Grupo Pulluicana-Quilquiñan (modificado de Timoteo, 2013). Localização dos afloramentos na Figura 18. 


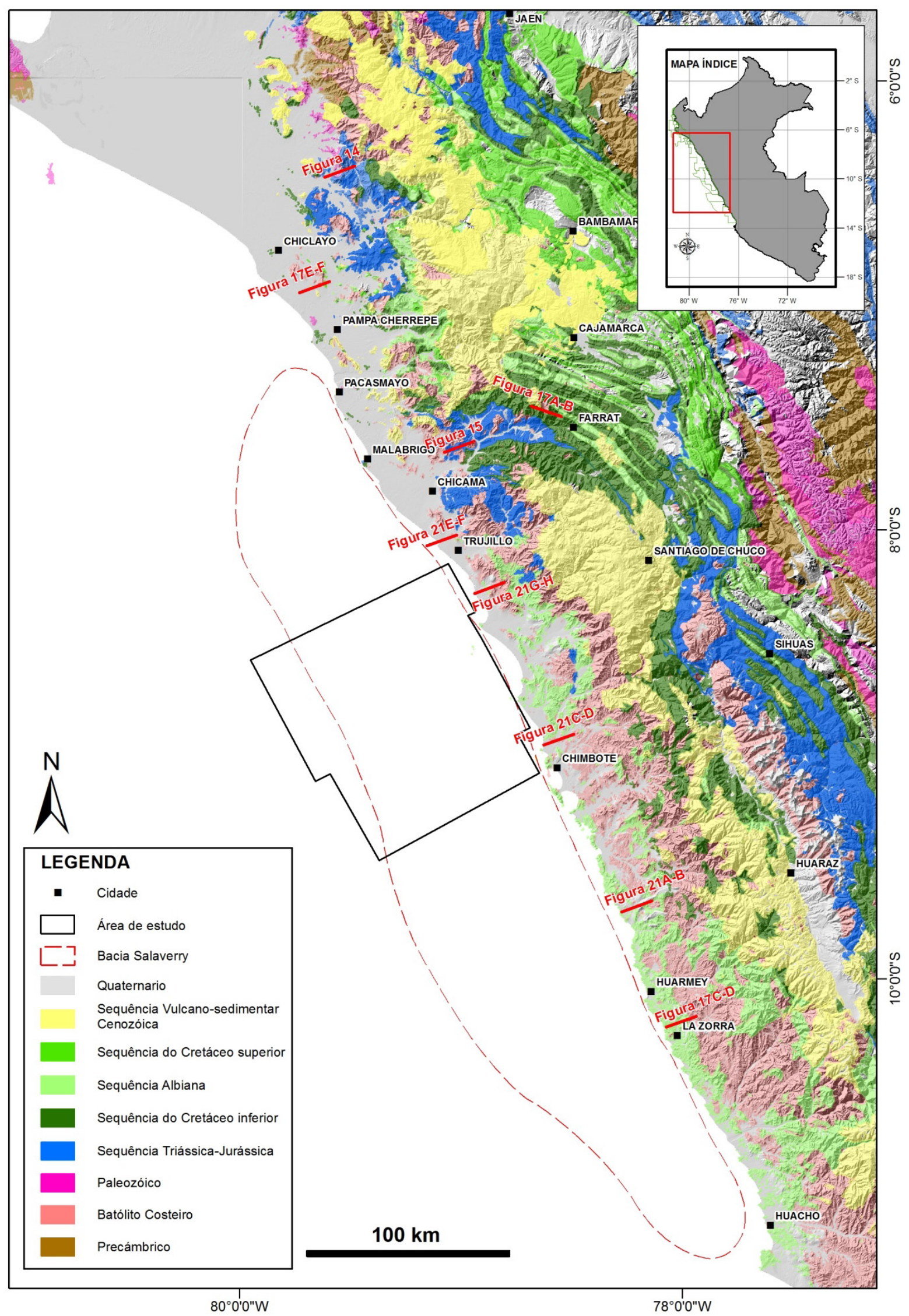

Figura 18. Mapa geológico simplificado do Peru com a disposição da Bacia Salaverry (delineada por tracejado vermelho) e a área de estudo (polígono preto), assim como a localização de afloramentos chaves. 


\section{SEQUÊNCIA CENOZÓICA}

A sequência Cenozóica compõe o preenchimento da Bacia Salaverry e sobrepõe-se ao embasamento Pré-Cenozóico. O seu registro estratigráfico é na sua maior parte advindo dos poços exploratórios Ballena-1X e Delfin-1X, e os afloramentos adjacentes à bacia localizados sobre a zona costeira.

As unidades mais velhas, correspondentes ao Eoceno inferior a médio, foram reconhecidas no poço Delfin-1X que ocorre no flanco leste da Bacia Trujillo. Já na Bacia Salaverry só há o registro a partir do Eoceno superior. É necessário mencionar que a nomenclatura estratigráfica formal para as unidades Cenozóicas na área de estudo não tem sido publicada ainda, portanto o presente trabalho segue, por correlação cronoestratigráfica, as subdivisões definidas na Província petrolífera do Noroeste do Perú (Bacias Talara, Sechura e Tumbes). Nesse contexto a sucessão sedimentar Eoceno-Holoceno é descrita a seguir.

\section{Eoceno}

$\mathrm{Na}$ base da sucessão reconhecem-se predominantemente arenitos quartzosos de grão médio a grosso intercalados com alguns níveis de siltitos e argilitos de coloração cinza e marrom, pertencentes à Formação Salina. Para o topo da seção no registro estratigráfico estão sobrepostos siltitos cinzas e marrons intercalados com argilitos cinzas e alguns níveis de calcários cinza claros, pertencentes à Formação Pariñas. Ambas as formações correspondem ao Eoceno inferior e só tem sido descritas no poço Delfin-1X (Figura 19). A Formação Terebratula composta principalmente por arenitos quartzosos de grão fino a muito grosso com intercalações de argilitos cinzas e siltitos cinzas e marrons, está depositada sobre as unidades litológicas da Formação Pariñas. Argilitos cinzas intercalados com siltitos marrons e cinzas, pertencentes à Formação Talara, ocorrem sobrepostos ao conjunto anterior. As formações Terebratula e Talara correspondem ao Eoceno médio e só tem sido perfuradas pelo poço Delfin-1X (Figura 19).

Para o topo da sucessão são reconhecidos argilitos de cor cinza a marrom intercalados com siltitos cinzas e marrons escuros e alguns níveis de calcários marrom claros, pertencentes à Formação Chira-Verdun no poço Delfin-1X. As unidades estratigráficas do Eoceno, como um todo, mostram uma sucessão ascendente de 


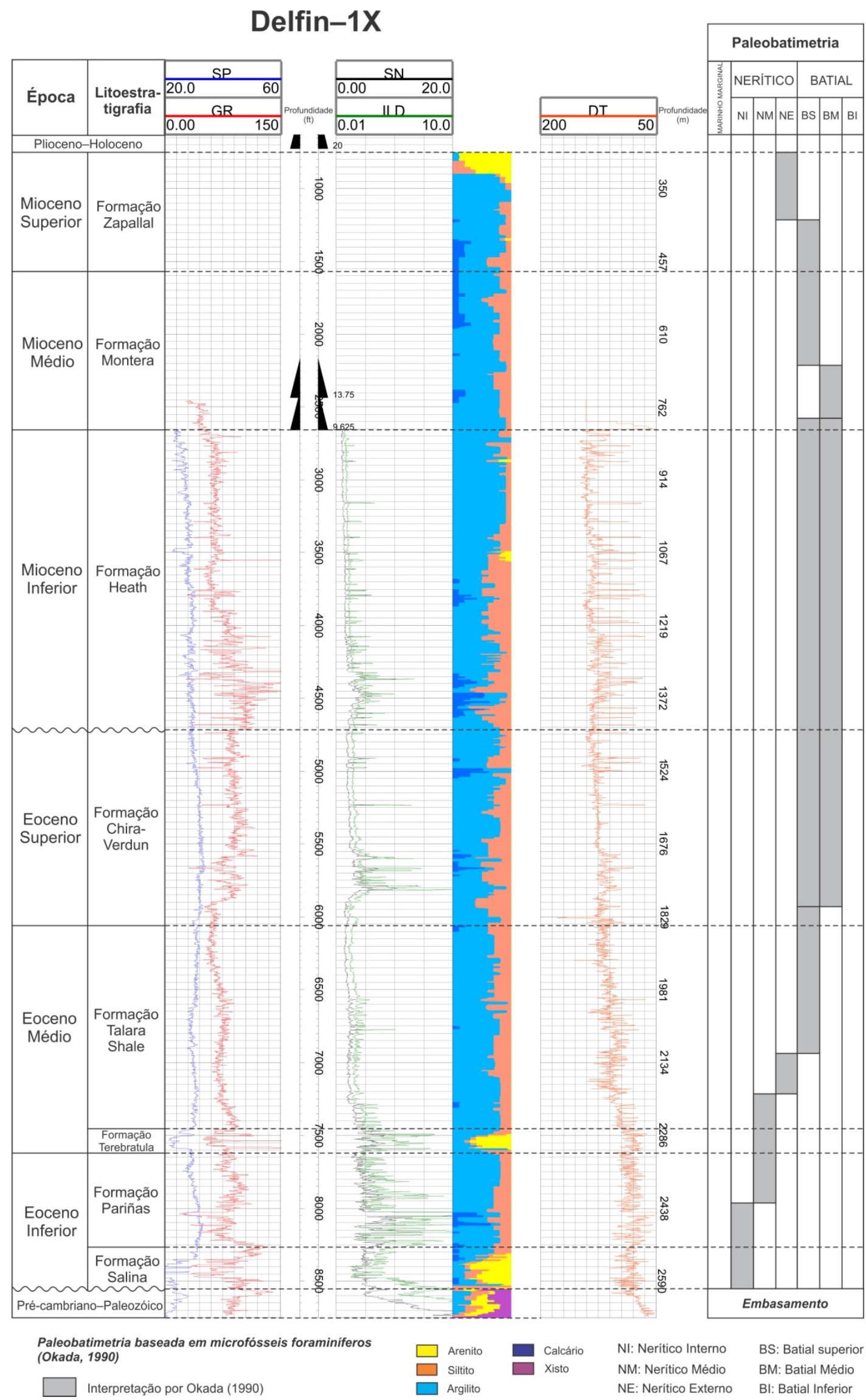

Figura 19. Perfil composto do poço exploratório Delfin-1X integrado com os dados de paleobatimetria. Profundidade em pés. 
aprofundamento, que começa num ambiente de plataforma interna até atingir um ambiente marinho profundo (Figura 19). Por outro lado, o poço Ballena-1X perfurou predominantemente arenitos quartzosos de grão fino com intercalações de argilitos cinza, pertencentes à Formação Verdun do Eoceno superior. Nessa locação a Formação Chira também do Eoceno superior encontra-se ausente (Figura 20).

\section{Oligoceno}

Ainda não há evidencias de registro sedimentar Oligoceno na bacia, o que sugere uma forte discordância regional. Os dados bioestratigráficos existentes não permitem diferenciar ainda depósitos remanescentes dessa idade na Bacia Salaverry (Figura 19 e 20).

\section{Mioceno}

Em ambos os poços exploratórios, da base para o topo se tem evidencias do Mioceno inferior, médio e superior.

A base da sucessão do Mioceno está representada predominantemente por argilitos cinzas com intercalações de siltitos cinzas e marrons, alguns níveis de arenitos quartzosos de grão fino e calcários marrons, as quais pertencem à Formação Heath do Mioceno inferior. Sobreposta às unidades anteriores, ocorre a Formação Montera do Mioceno médio que é composta de: argilitos de coloração cinza e marrom intercalados com siltitos marrons, alguns níveis de calcários cinzas escuros e marrons claros e arenitos quartzosos de grão fino. Em direção ao topo da sucessão do Mioceno são reconhecidos argilitos cinzas intercalados com siltitos cinzas, alguns níveis de arenitos quartzosos de grão fino, arenitos conglomeráticos e calcários marrons claros, todos pertencentes à Formação Zapallal do Mioceno Superior (Figura 19 e 20).

A sucessão do Mioceno, como um todo, representa um ciclo completo de variação de fácies de sedimentação rasa para profunda, que atinge a máxima inundação no topo do Mioceno inferior em um ambiente marinho profundo. A partir desse ponto, inicia-se o trato de sistemas de mar alto que atinge o topo do Plioceno, com sedimentação rasa em ambiente de plataforma. (Figura 19 e 20). 


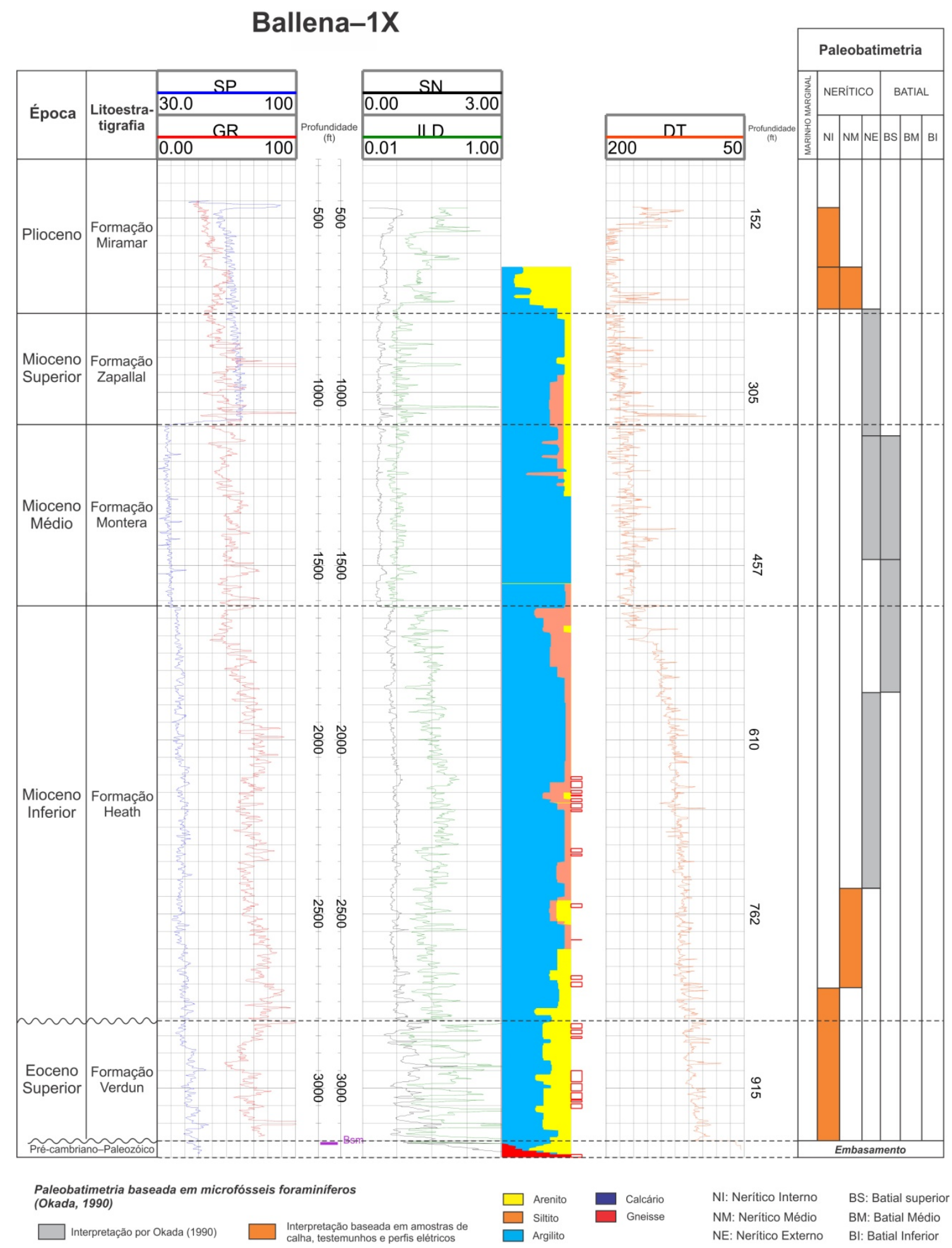

Figura 20. Perfil composto do poço exploratório Ballena-1X integrado com os dados de paleobatimetria. Profundidade em pés. 


\section{Plioceno}

Em ambos os poços só se tem evidencias da seção basal da sucessão do Plioceno, a qual consiste de arenitos conglomeráticos intercalados com siltitos cinza (Figura 20). Por outro lado, a secção de maior espessura da sucessão do Plioceno ainda não tem sido perfurada na bacia e só tem sido identificada nas seções sísmicas interpretadas.

\section{Plesitoceno - Holoceno}

O registro sedimentar de idade Plioceno-Holoceno não tem sido perfurado na bacia, mas é caracterizado por diferentes depósitos sedimentares que possuem exposição nos afloramentos remanescentes adjacentes à bacia, sobrepostos às rochas cretácicas que formam o embasamento Pré-Cenozóico na zona costeira (Figura 21).

Com base no trabalho de campo realizado na presente pesquisa foi possível reconhecer diferentes depósitos sedimentares sobre a zona costeira nos afloramentos adjacentes à área de estudo, entre as localidades de Chimbote e Trujillo. Nesse contexto a sucessão sedimentar Pleistoceno-Holoceno é descrita a seguir, segundo as estações de trabalho.

Estação Punta Las Aldas: No extremo sul da área de estudo, na estação Punta Las Aldas (Fig. 18), da base para o topo se reconheceram camadas de conglomerados que possuem geometria de canais com base erosiva, intercalados com siltitos e folhelhos que possuem geometria de lençóis subparalelos de grande continuidade lateral que formam as planícies de inundação. Esses afloramentos correspondem a depósitos fluvio-aluviais com espessura entre 30 e $50 \mathrm{~m}$, que se sobrepõem discordantes às andesitas e piroclastos do Grupo Casma (Figura 21A e B).

Estação Chimbote: Sob a parte central da área de estudo, na estação Chimbote (Fig. 18), se reconheceram da base para o topo arenitos de grão médio a grosso com laminações onduladas e ripples, intercalados com camadas de arenitos massivos de grão muito grosso. Sobreposta as litofacies anteriores ocorrem arenitos com estratificação cruzada tangencial. Esses afloramentos correspondem a depósitos de antepraia (shoreface) e praia (foreshore) com espessura entre 10 e $15 \mathrm{~m}$, e a sua base não esta exposta (Figura 21C e D). 

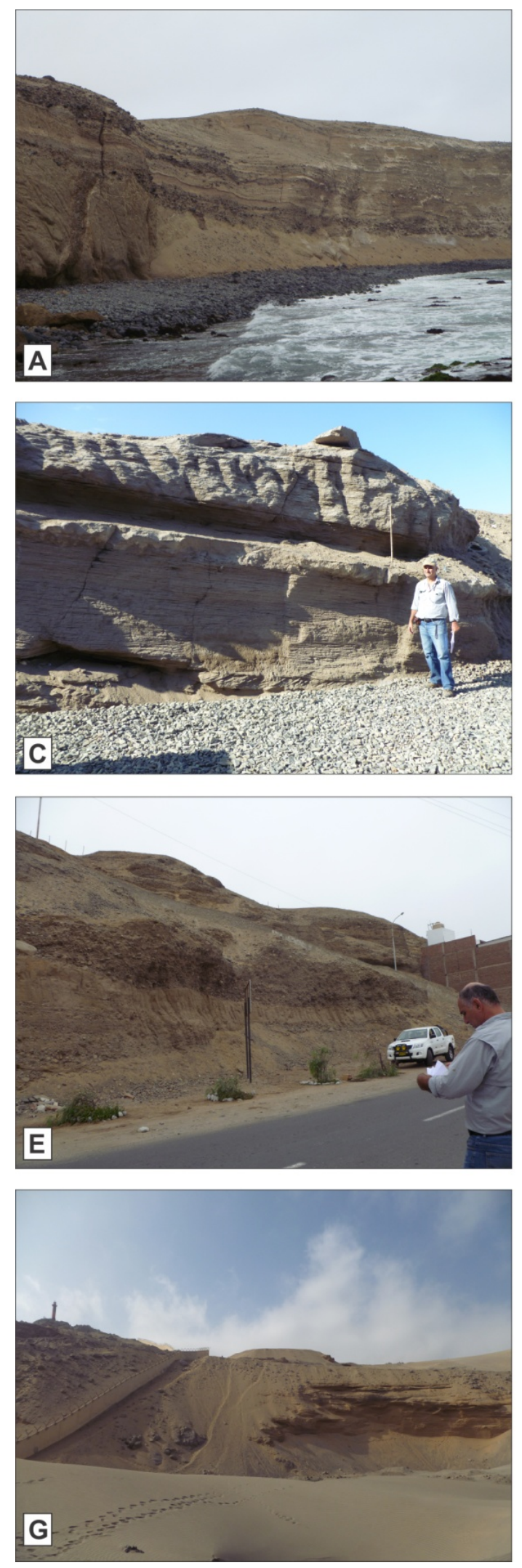
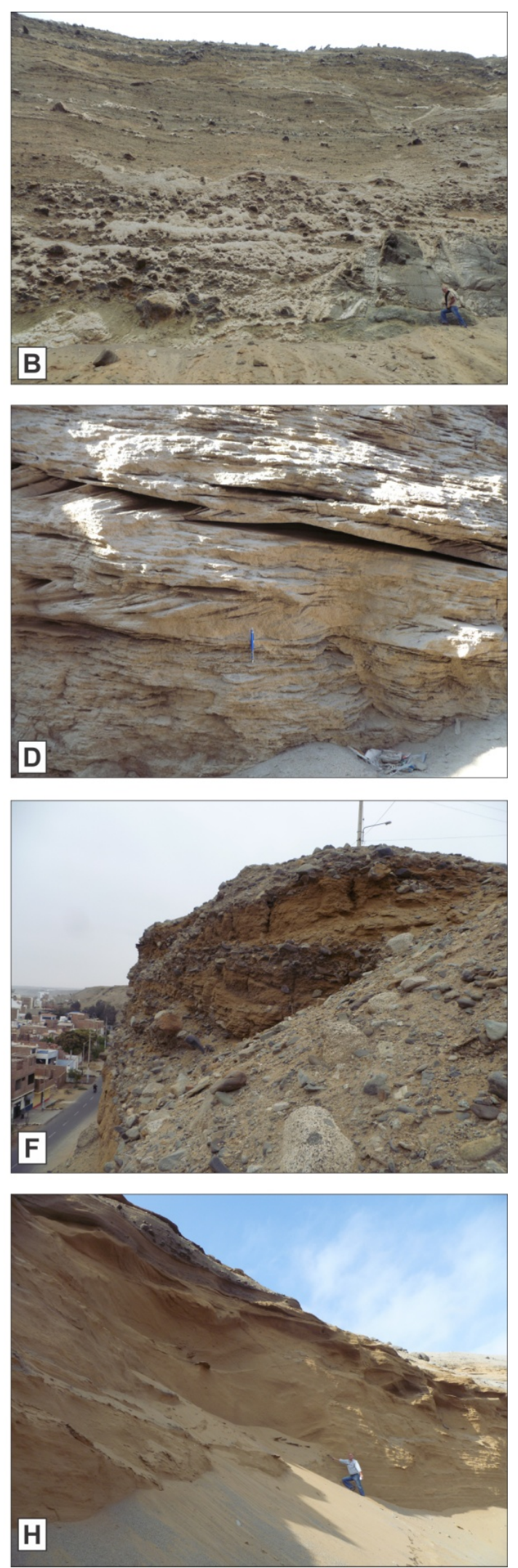

Figura 21. Vista panorâmica e de detalhe dos afloramentos de idade Plioceno-Holoceno reconhecidos no trabalho de campo. A) e B) Depósitos fuvio-aluviais que se sobrepõem discordantes às rochas vulcânicas do Cretácico superior. C) Depósitos de antepraia e praia. D) arenitos com estratificação cruzada tangencial. E) e F) Depósitos de leques aluviais. G) e H) Depósitos eólicos que se sobrepõem discordante aos granitos do Batólito Costeiro. 
Estação Huanchaco: Na estação Huanchaco (Fig. 18), da base para o topo se reconheceram camadas de conglomerados e arenitos grossos que possuem geometria de canais com base erosiva, intercalados com siltitos e folhelhos que possuem geometria de lençóis subparalelos de relativa continuidade lateral. Tais facies sedimentares tornam-se cíclicas para o topo da sucessão, predominando os conglomerados. Esses afloramentos correspondem a depósitos de leques aluviais com espessura entre 20 e $30 \mathrm{~m}$, e a sua base não esta exposta (Figura 21E e F).

Estação Salaverry: No extremo norte da área de estudo, na estação Salaverry (Fig. 18), se reconheceram arenitos finos a grossos com estratificação cruzada de grande porte intercalados com lençóis de areia. Tais facies sedimentares tornam-se cíclicas para o topo da sucessão. Esses afloramentos correspondem a depósitos eólicos com espessura entre 40 e 60 m, que se sobrepõem a os granitos do Batólito da costa (Figura 21G e H).

É necessário mencionar que os depósitos sedimentares descritos acima podem ser mais velhos do que Plioceno, mas se precisam de novos dados bioestratigráficos e datações que permitam conhecer com precisão a sua idade. 


\title{
CAPÍTULO 3: TECTONO-SEDIMENTARY EVOLUTION OF THE
} SALAVERRY BASIN, PERUVIAN ANDES FOREARC: NEW INSIGHTS IN ASSESSING POTENTIAL RESERVOIRS AND

\section{PLAYS}

Diego Timoteo ${ }^{\text {a, }}$; Farid Chemale Junior ${ }^{\text {a,b }}$; Edgar Borda ${ }^{c}$, Andréa Ritter Jelinek ${ }^{\text {d }}$; Christie Helouise Engelmann de Oliveira ${ }^{\mathrm{d}}$

\footnotetext{
a Instituto de Geociências, Universidade de Brasília, Brasília, Brazil

${ }^{b}$ Programa de Pós-Graduação em Geologia, Universidade do Vale do Rio dos Sinos, São Leopoldo - RS, Brazil

c Savia Peru S.A., Lima, Peru

d Instituto de Geociências, Universidades Federal do Rio Grande do Sul, Porto Alegre, Brazil
}

\begin{abstract}
This study is focused in Salaverry Basin, Peruvian Andes Forearc, which contains three oil seeps sampled and analyzed, suggesting at least one active petroleum system inside the basin. Seismic stratigraphic analysis calibrated with available well and core data, and integrated with Apatite fission track (AFT) analysis was performed in order to provide the spatial-temporal evolution of Cenozoic depositional systems in this basin. It has been developed in extensional tectonic setting punctuated by compressive periods controlled by the interaction between Nazca and South American plates, and Nazca ridge subduction. As a result, the Cenozoic succession in the central portion of Salaverry Basin is best subdivided into eleven seismic stratigraphic sequences (S1$\mathrm{S} 11$ ), controlled predominantly by tectonism and also by sea-level fluctuations. Indeed, this work adds new potential reservoir rocks in the S1, S2, S4, S5, S7 and S8 sequences; and seal rocks in the S3, S6, S7 and S9-S11 ones for the petroleum systems previously defined in the basin. Fission track thermochronology analysis on apatite suggest a main uplift event in the basin at ca. $25 \mathrm{Ma}$, which triggered the erosion of Oligocene rocks and the uppermost section of the Upper Eocene deposits, and is correlated with the Incaic III tectonic phase. These new petroleum system elements are related to the proposed potential plays, which in turn through a follow-up assessment would become in very attractive exploratory prospects in Salaverry Basin central portion.
\end{abstract}

Keywords: Salaverry Basin, Seismic stratigraphy, Hydrocarbon exploration, Plays, Forearc Basin, Peruvian margin, Andes. 


\subsection{INTRODUCTION}

On the western margin of South America are developed the Peruvian Forearc basins, which can be divided into two segments. The Northwest petroliferous province (Talara, Sechura and Tumbes Basins), which has been the focus of exploration and production for more than 100 years; and the south-central province (Salaverry and Trujillo Basins), which has been relatively unexplored (Fig. 1) (see Timoteo, 2013 for an overview). In this context; the existence of world-class Cenozoic reservoirs in Northwest Peru has motivated its exploration to the south, where recent works have increased the geological knowledge of this exploratory province (Romero et al., 2013; Valencia et al, 2013; Timoteo et al., 2014).

This study is focused in Salaverry basin; which contains several oil seeps and oil/gas shows from nearest wells suggesting at least one active petroleum system inside the basin, as yet sparsely tested by exploratory wells (Fig. 1).

Previous works identified and characterized potential Cenozoic reservoirs based on petrophysical studies from well data, and sedimentological/petrographic studies from outcrops adjacent to the basin along the coast (Minguito et al., 1997; Wine et al., 2001; Peña, 2011). Therefore, the present work proposes a seismic stratigraphic analysis that integrates all the datasets mentioned before.

In Salaverry Basin, only two wells pass through the Cenozoic rocks. Ballena-1X exploratory well is located at western margin over a prominent structural high (Paracas Arch), and Delfin-1X exploratory well was drilled at eastern flank of Trujillo Basin (Fig. 2). Both wells penetrated sedimentary successions and reached the metamorphic basement. Moreover, cores in these wells are limited and correspond only to the lower interval of Ballena-1X well.

In order to provide regional maps of seismic facies and the spatial-temporal evolution of Cenozoic depositional systems in the basin, we applied seismic stratigraphy principles and seismic facies analysis of 2D and 3D seismic data calibrated to wells and core data available (Mitchum et. al., 1977; Sangree et al., 1977; Vail et al., 1977, 1987; Veeken, 2013). Our studies, allow to present an updated interpretation of the regional seismic stratigraphy of the Cenozoic units in central portion of Salaverry Basin and a new insight on their petroleum systems. 


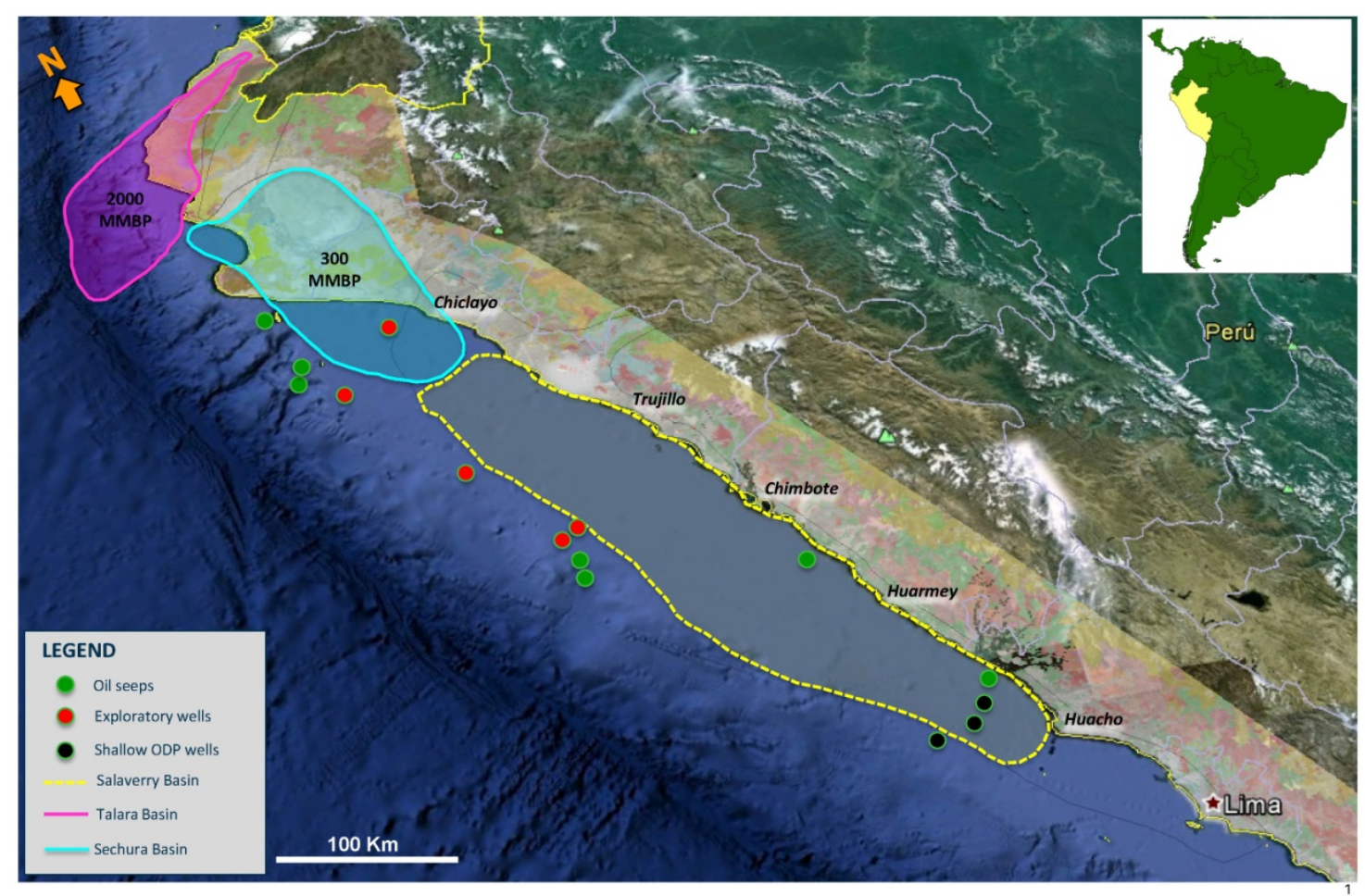

Figure 1. Location map of the Peruvian Forearc petroliferous/tectonic province, comprising the Salaverry Basin (yellow dotted line) and Northwest petroliferous province (Talara and Sechura Basins). The exploratory wells and oil seeps from south-central province are also indicated (Modified from Timoteo et al., 2014).

\subsection{DATA AND METHODS}

\subsubsection{Seismic and well log data}

This project is based on regional 2D seismic surveys that accumulate 2831 linear $\mathrm{Km}$ covering approximately $11,600 \mathrm{~km}^{2}$ of basin area, 3D seismic survey covering 364 $\mathrm{km}^{2}$, and two exploratory wells (Fig. 2). The 2D seismic lines are oriented parallel and perpendicular to the Peruvian coast. Where possible time shifts between individual seismic lines have been corrected. Based on seismic acquisition parameters the average vertical and lateral resolutions of $2 \mathrm{D}$ seismic surveys are estimated in $\sim 40 \mathrm{~m}(\lambda / 4)$ and $\sim 80 \mathrm{~m}$, respectively. The seismic surveys are time migrated thus the vertical scale and thickness maps are in milliseconds two way time (ms TWT). Seismic imaging is generally good but quality and resolution decrease over the western margin and where shallow and deep gas indicators are observed.

Only two exploratory wells were drilled on western margin of Salaverry Basin. Although these wells are relatively old, year 1971, they have a standard wireline log suite comprising gamma ray (GR in API units), spontaneous potential (SP in mv), sonic 
(DT in $\mu \mathrm{s} / \mathrm{ft}$ ), density (DEN in $\mathrm{g} / \mathrm{cm} 3$ ) and resistivity (ILD and SN in ohm.m units) logs. Cuttings and core samples, and biostratigraphic descriptions are utilized to support interpretation of depositional environments. Stratigraphic age of Cenozoic deposits are based on a combination on micropaleontological, calcareous nannoplankton, diatomaceous and *OEMA analyses (Valdespino and Seminario, 1976a, 1976b; Schell, 1978; Okada, 1990; Schrader and Cruzado, 1992; Canchaya and Paz, 1992; Narvaez and Pardo, 1993; Morales and Gonzales, 2006). Likewise sonic and density logs, and check-shot survey were used to generate synthetic seismograms in order to perform seismic well tie.

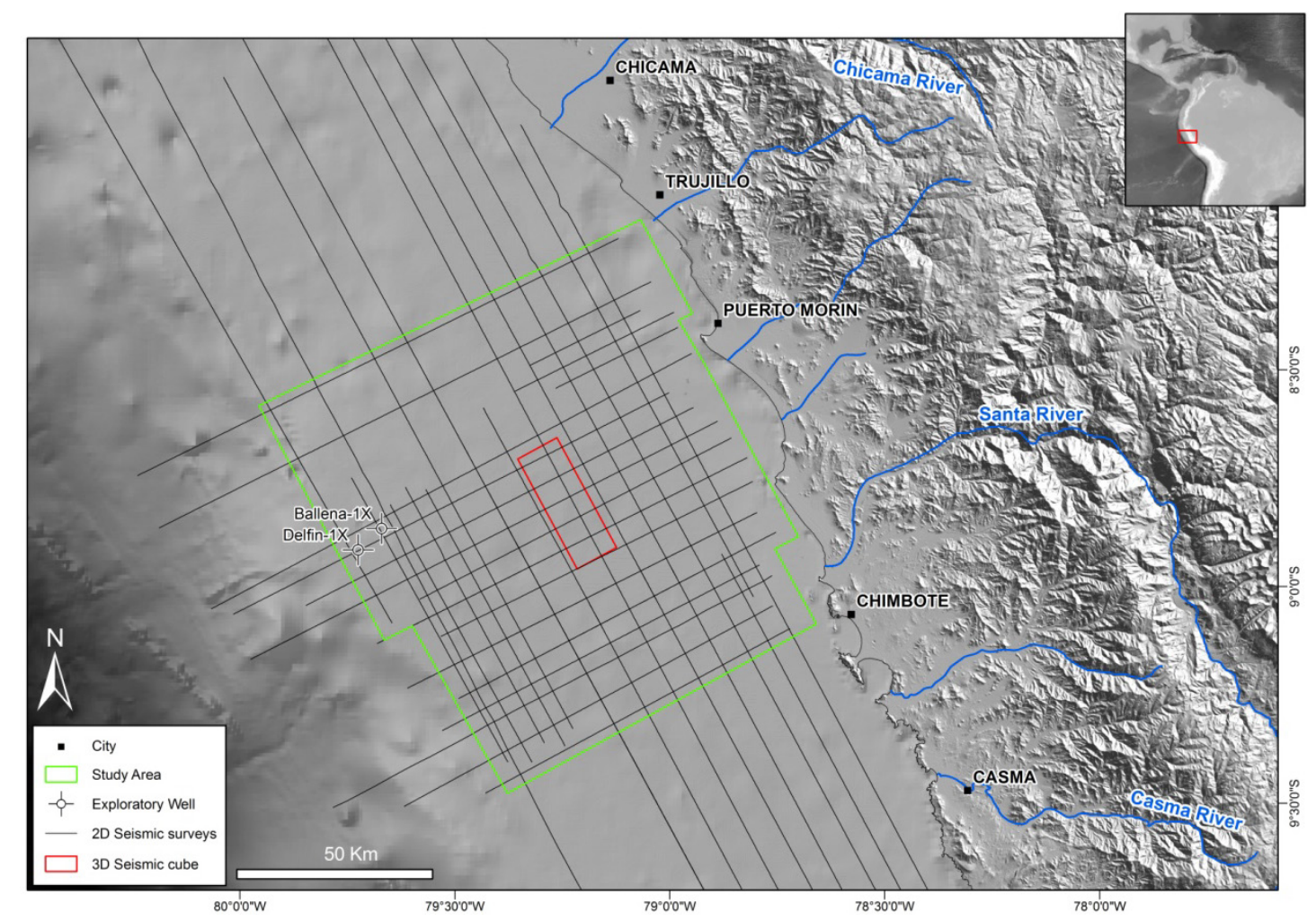

Figure 2. Map of the northern central Peruvian margin showing the location of the study area (green polygon) and its relation with the main fluvial system. The figure also illustrates the location of 2D and 3D seismic surveys, and the exploratory wells used in the present project.

In addition, surface data correspond to field geological reconnaissance carried out in outcrops along the coast and adjacent to the basin, where Upper Cenozoic remaining deposits overlay Cretaceous-Paleogene economic basement.

This study develops a seismic stratigraphic framework for the Cenozoic units in Salaverry Basin following standard seismic stratigraphic interpretation workflow (Mitchum et. al., 1977; Sangree et al., 1977; Vail et al., 1977, 1987; Veeken, 2013). 
Reflection terminations (e.g. onlap, downlap, toplap, offlap, apparent toplap and erosional truncation) are identified and mapped in order to define key seismic horizons (seismic discontinuities) that bound major seismic stratigraphic sequences across the basin. In the same way; seismic facies are recognized, described and mapped per each seismic stratigraphic sequence; based on reflection configuration (geometry), continuity, amplitude, frequency and the overall exterior shape of the reflection package (Table 1).

*OEMA: Spanish acronym of "Other elements of Animal Matter".

\subsubsection{Thermocronological analysis}

In order to better understand deposition and uplift history in Salaverry Basin, the seismic interpretation was integrated with Apatite Fission Track (AFT) analysis. We collected two sedimentary rock samples, fine-grained sandstone and conglomeratic sandstone, from Ballena-1X well thereby providing the samples for AFT analysis (Figs. 2 and 4). The apatite fission track thermochronology has been carried out at the Federal University of Rio Grande do Sul (UFRGS) in Brazil. Samples were crushed and milled using jaw crusher. Then, the apatite grains were separated by conventional procedures using heavy liquids and a sand magnetic separator after concentration by hand panning.

The apatite mounts were covered with mica sheets to obtain the fission track ages using external detector method (Gleadow, 1981). Neutron irradiation was carried out at the FRM-II reactor in Garching (TU München, Germany). The mica detectors were revealed by etching in $48 \% \mathrm{HF}$ for $18 \mathrm{~min}$. Fission-track counting was performed at a Leica Microscope under 1000x magnification. Fission track ages were calculated using the zeta calibration approach (Hurford and Green, 1982, 1983) with CN5 dosimeter glass and Durango apatite age standard. The ages are expressed as central ages with percent variation (Galbrait and Lasllet, 1993). Based on the distribution of AFT singlegrain ages, confined track lengths, kinetic indicators as Dpar and independent geological constraints; the temperature-time $(\mathrm{T}-\mathrm{t})$ path for each sample was modeled using the HeFTy program (Ketcham, 2005) and the multi-kinetic annealing algorithm of Ketcham et al. (2007).

The constraints for inversion modeling used are: (a) an initial constraint was set at $200 \pm 20^{\circ} \mathrm{C}$ at a time span older than the corresponding apatite apparent age; (b) a constraint was set in a large span of temperature $\left(\sim 80-20^{\circ} \mathrm{C}\right)$ at the $t$ of the oldest AFT 
grain age ( 180 Ma); (c) a large T-t box was imposed with $\mathrm{T}$ closed limits of the AFT PAZ temperature $\left(140-60^{\circ} \mathrm{C}\right)$ at a time corresponding the mean apparent age; and $(\mathrm{d}) \mathrm{a}$ present day temperature provided the final modeling constraint.

\subsection{GEOLOGICAL SETTING}

The study area is part of Forearc domain, which comprises a large petroliferous/tectonic province in the western margin of South America over the Peruvian sea (Fig. 1). This area is located in Salaverry Basin, offshore central Peru between latitudes $8^{\circ} \mathrm{S}-9^{\circ} 30^{\prime} \mathrm{S}$, and it is controlled by the interaction between Nazca and South American plates, the Andean Orogeny and Nazca ridge subduction (Fig. 2) (Kulm et al., 1981; Azalgara, 1993; Benavides, 1999; Aleman and Ramos, 2000; Decelles, 2009; Ramos, 2009, 2014).

The geological evolution of the Peruvian margin is complex since it contains those rocks formed during the Andean Cycle and basement rocks. The Pre-Cenozoic basement rocks consist of (i) Precambrian metamorphic rocks, which are interpreted to be part of the Paracas Terrane, (ii) Ordovician granitoids of San Nicolás Batholith, which are interpreted to formed in an active subduction setting, (iii) a Carboniferous sedimentary package of shales and sandstones deposited in fluvial-deltaic environment, (iv) Permian gabbro-diorites described in the basal section of San Miguel-1X exploratory well. The second and fourth units described above, are interpreted to formed in an active forearc setting in the Peruvian margin during the Ordovician-Permian, and thus suggest that remaining magmatic arc and associated sedimentary deposits would be preserved offshore. (v) Jurassic-Cretaceous sedimentary and volcano-sedimentary sequences formed also in active forearc setting dominated by extensional tectonics that configured the entire margin in grabens and half-grabens filled out with deep marine and shallow water sedimentary deposits associated to the eastern volcanic arc; and (vi) Upper Cretaceous to Paleogene granitoids belongs to the Coastal Batholith. In this way, the Cenozoic Salaverry Basin has been developed over the Paleo-Mesozoic Peruvian continental margin basin (Myers, 1974, 1980; Guevara, 1980; Gaffney-Cline, 2005; Reitsma, 2012; Timoteo et al., 2012; Pfiffner and Gonzales, 2013; Romero et al., 2013; Timoteo, 2013; Ramos, 2014). 


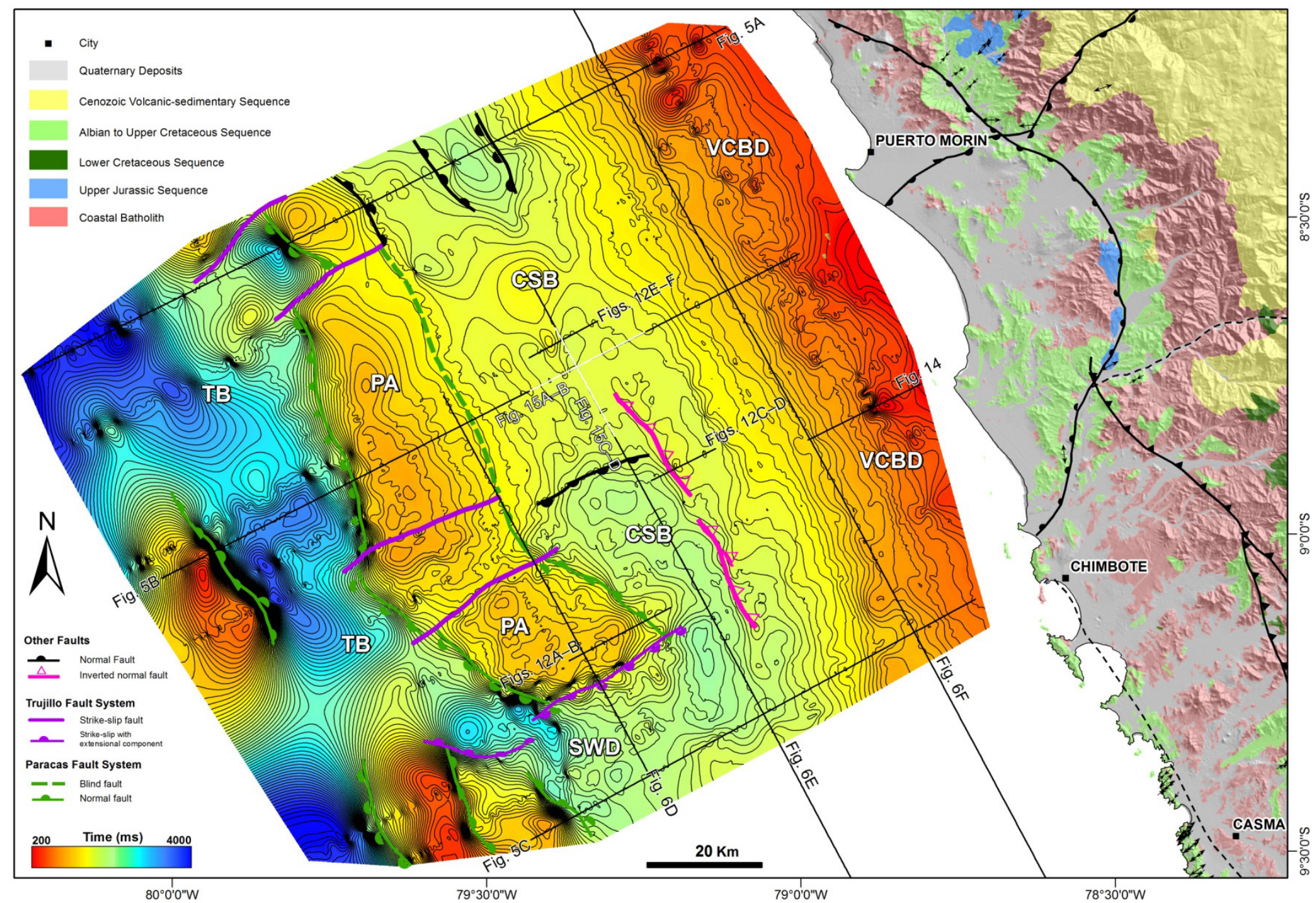

Figure 3. Time structure map of the Top Pre-Cenozoic Basement showing the main fault systems and the morphostructural domains in the study area. Contour lines are every $50 \mathrm{~ms}$. TB $=$ Trujillo Basin; PA = Paracas Arch; CSB = Cenozoic Salaverry Basin; VCBD = Volcanic arc and Coastal Batolith Domain, $\mathrm{SWD}=$ Southwestern Depocentre. Also it illustrates the onshore geological settings. 
In Salaverry Basin, the Cenozoic stratigraphic record reaches $\sim 2 \mathrm{~km}$ thickness and is controlled by the subduction processes related to the Andean-type Orogenic cycle and locally by the interaction with the Nazca Ridge and the Paracas Arch (Fig. 3). This Eocene-Holocene succession is derived from data of Ballena-1X and Delfin-1X exploratory wells, and coastal outcrops preserved onshore. The Pre-Cenozoic Basement (PCB) is composed by rocks of different age and lithology along the basin, as it mentioned above. This economic basement underlay the entire Cenozoic sequence (Fig. 4).

The Upper Eocene Verdun Formation unconformably overlies the PCB, and it is composed predominantly by fine-grained quartz sandstones interbedded with claystones; deposited in deltaic/shelf environment. Following upward in the section and unconformably lies the Lower Miocene Heath Formation; which is composed by finegrained quartz sandstones at the base, followed by gray claystones interbedded with gray to brown siltstones. As a whole, this unit shows a deepening upward succession from deltaic/shelf to deep marine environment.

Then, unconformably overlies the Middle Miocene Montera formation. This unit is composed predominantly by brownish gray and grayish brown claystones interbedded with brown siltstones and some layers of fine-grained quartz sandstones; deposited in deep marine environment. Later and unconformably lies the Upper Miocene Zapallal Formation, which is composed predominantly by gray claystones interbedded with very fine sandstones and gray siltstones; deposited in outer shelf environment.

Towards the top of the succession, in apparent unconformity lies the PliocenePleistocene sedimentary succession, which is composed by Miramar and Hornillos Formation. The basal section of Miramar Formation consists of conglomeratic sandstones interbedded with some layers of gray claystones; deposited in shallow marine environment. Moreover, the thickest part of this succession has not been drilled yet and is located in the basin foredeep. Furthermore, this unit has been recognized onshore as fluvial-alluvial, shoreface and aeolian deposits distributed along the coast.

The formal stratigraphic nomenclature for the Cenozoic units in the study area has not been published. Therefore the present work follows the stratigraphic subdivisions of Dunbar et al. (1990), Minguito et al. (1997), Martinez et al. (2005), Deckelman et al. 
(2008), and Gonzales (2014); which are based on more recent seismic, well, field and biostratigraphic data.

The Eocene to Holocene siliciclastic succession has been fed by major fluvial systems from eastern Andean region such as Santa, Casma and Chicama rivers (Fig. 2).

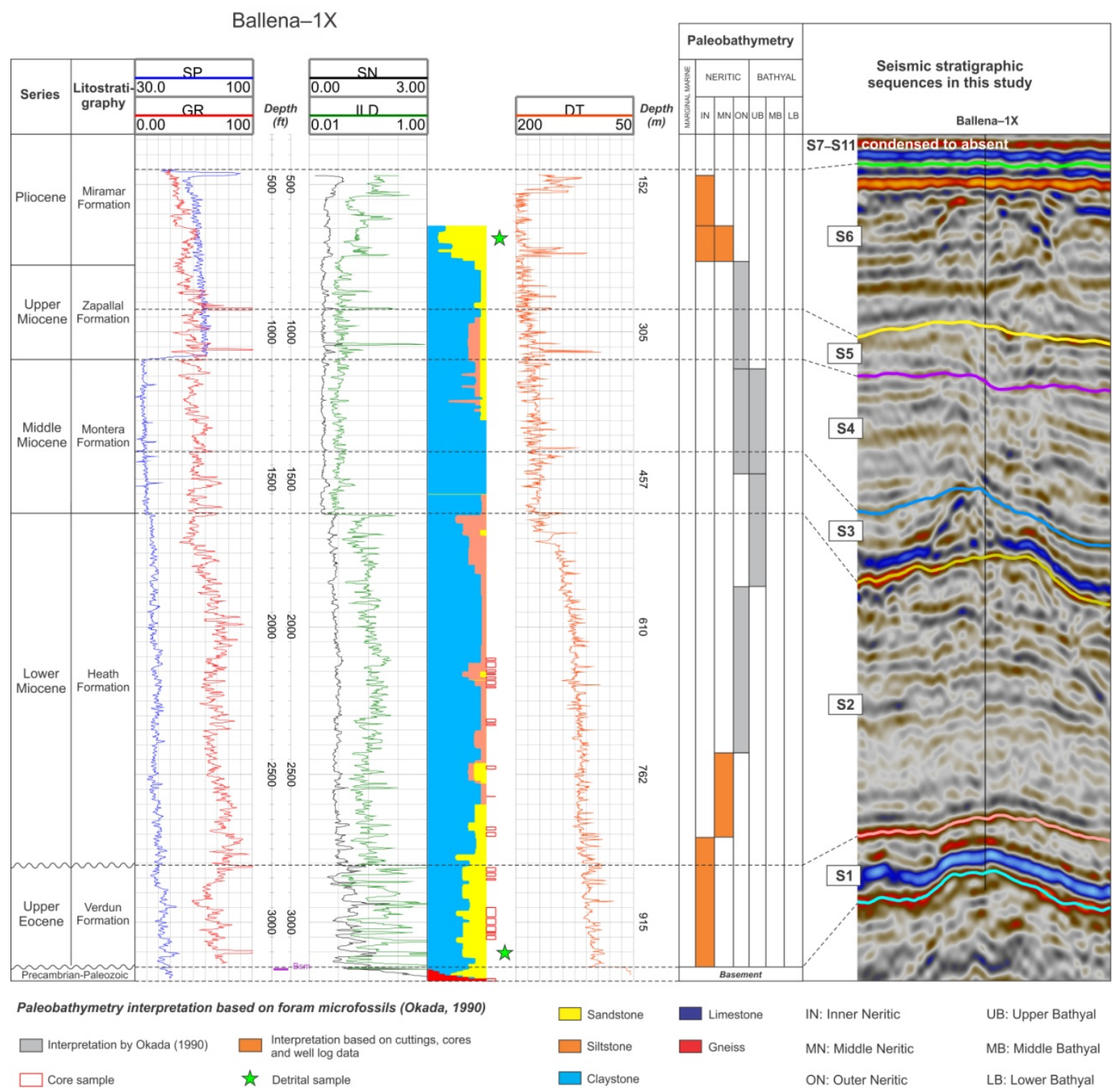

Figure 4. Litostratigraphic subdivisions of the Cenozoic succession in the central portion of Salaverry Basin and the corresponding seismostratigraphic units interpreted in this study.

The Peruvian Offshore southward of the Northwest petroliferous province (Talara, Sechura and Tumbes basins) has been relatively unexplored. Several oil seeps have been recognized, sampled and analyzed, suggesting different active petroleum systems; as yet sparsely tested by exploratory wells (Valencia et al., 2013; Timoteo et al., 2014). 


\subsection{STRUCTURAL FRAMEWORK}

Cenozoic Salaverry Basin has been developed in extensional tectonic setting punctuated by compressive periods over Paleo-Mesozoic rocks that conform the ancient Peruvian continental margin basin; which evolved in extensional tectonic context associated to eastern volcanic arc. In this context, Salaverry Basin consists of depocentres and structural highs developed during Eocene-Pleistocene collision between Nazca-Farallon and South American Plates. The central portion of Salaverry Basin is located almost entirely offshore with few remaining Upper Cenozoic deposits exposed in outcrops along the coast between Chimbote and Trujillo cities (Fig. 3).

Two major depocentres were recognized, Trujillo Basin to the west and Salaverry Basin to the east. Both basins are limited by a prominent structural high known as Paracas Arch and they are linked only in the southern part of the study area (southwestern depocentre). Trujillo is deeper than Salaverry due to basin formation and fill began before during Early Eocene. The Paracas Arch acts as current western margin of Salaverry Basin whereas the coastline acts as current eastern flank. Over the eastern margin, concentric morphological features of several sizes along NW-SE trend correspond to offshore extension of volcanic arc and Coastal Batholith domain (Fig. 3). These features correspond to volcanic cones and intrusive bodies and act as shallow structural highs deforming ancient rocks or truncating younger deposits (Fig.14).

Major NW-SE trending normal faults with east and west vergence conform the Paracas fault system. Some of them cut the PCB and younger rocks while others correspond to blind faults that don't reach the Cenozoic sequence and therefore has subtle evidence in the structural contour map of the top of PCB (Fig. 3). In the same way, major NE-SW trending strike-slip faults conform the Trujillo fault system. They correspond mainly to sinistral and dextral strike-slip faults. In addition, strike-slip faults with extensional component are recognized in the southwestern depocentre margins (Fig. 3). On the other hand, several smaller faults are present in the Cenozoic Salaverry Basin. Extensional faults with east and west vergence cut the Cenozoic sequence and some of them reach the economic basement. Moreover, Miocene inverted normal faults generate fault-propagation folds as intrabasinal highs due to local compressive events product of Nazca Ridge subduction beneath South American plate (Figs. 3 and 12C-F). 


\subsection{SEISMIC STRATIGRAPHY OF THE CENOZOIC SUCCESSION}

Twelve seismic sequence boundaries are identified within the Cenozoic succession based on reflection terminations (Figs. 4, 5 and 6). These seismic sequence boundaries, together with major changes in seismic facies, define eleven seismic stratigraphic sequences: S1 - S11. The following section describes the key seismic horizons and the seismic sequences they define.

\section{Seismic Sequence S1}

The S1 second-order sequence is bounded at its base by SB1 seismic horizon (cyan) and at its top by SB2 seismic horizon (salmon) (Figs. 4, 5 and 6). It comprises the Verdun Formation ranging in age within the Upper Eocene $(\sim 41.2-33.9 \mathrm{Ma})$ (Fig. 4). In the southwestern depocentre Middle Eocene deposits are recognized, but poor quality seismic data preclude detailed seismic interpretation thus they are considered as part of S1 (Fig. 5C). Towards the western margin of Salaverry Basin, S1 seismic sequence onlaps on the Paracas Arch to the north and, on the PCB to the south (Figs. $5 \mathrm{~B}, 5 \mathrm{C}$ and 7$)$. The $\mathrm{SB} 1$ reflection is continuous and characterized by a strong peak that coincides with the top of the PCB. The SB2 seismic horizon is relatively continuous and composed by a moderate peak that coincides with a regional unconformity (Fig. 4).

The S1 geographic extent is limited to the western margin and to the southwestern depocentre, due to $\mathrm{S} 1$ sequence is mainly developed in Trujillo Basin. The S1 Isochron map shows major thickness in the southwestern depocentre $(400-940$ ms TWT) whereas over the Paracas Arch, the S1 seismic sequence is generally thin $(<50 \mathrm{~ms}$ TWT) or absent (Fig. 8). The bulk of S1 sequence consists of hummocky seismic facies (SF10) that drape the SB1 topography as a wedge. To the east, SF6 seismic facies pass transitionally to hummocky reflections before described (Fig. 10). To the SE, S1 sequence is condensed and sheet drapes of discontinuous reflections (SF6) are recognized. On the southwestern depocentre, the S1 sequence is predominantly characterized by SF4 seismic facies. In the same depocentre, seismic facies are unclear due to seismic quality decreases by the presence of different DHIs (Direct Hydrocarbon indicators). Also westward, the $\mathrm{S} 1$ is punctuated by chaotic/contorted seismic facies (SF11) (Fig. 10, Table 1). 


\section{Seismic sequence $\mathbf{S 2}$}

The S2 second-order sequence is bounded at its base and top by SB2 (salmon) and SB3 (light yellow) seismic horizons, respectively. In addition, SB2 has limited distribution thus SB2 and SB1 merges and thereby conforms the base of this sequence towards the east (Fig. 7). Litostratigraphically, it comprises the Heath Formation ranging in age within the Lower Miocene ( $23.1-15.9 \mathrm{Ma})$ (Fig. 4). The S2 sequence onlaps the SB1 seismic horizon mainly over the Paracas Arch in the western margin (Fig. 7), and is absent on structural highs in southern part of the basin (Fig. 12A and B). Based on biostratigraphic, well log and seismic data; the SB3 seismic horizon is a local unconformity on the western margin while is conformable over the deepest parts of the basin (Figs. 4 and 5C). The S2 Isochron map shows wider geographic extent than the S1 sequence. The S2 is thickest over the southwestern depocentre, where it reaches up to $450-540 \mathrm{~ms}$ TWT. On structural highs in the south part of the study area, S2 is generally thin $(<80 \mathrm{~ms}$ TWT); and similar to the S1 seismic sequence is very thin to absent $(<30 \mathrm{~ms}$ TWT) to the east and north (Fig. 8). The bulk of S2 seismic sequence is characterized by hummocky seismic facies (SF10). To the east, the S2 is condensed in part and passes transitionally to SF6 reflections. In the southwestern depocentre; SF4 are the dominant seismic facies. At the same area and specifically on the fault zones the seismic resolution is poor, thus the seismic facies are unclear. Westward, hummocky reflections pass laterally to chaotic/contorted reflections (SF11) (Fig. 10, Table 1).

\section{Seismic sequence $\mathrm{S3}$}

The S3 third-order sequence is bounded at its base by SB3 seismic horizon (light yellow) and at its top by SB4 seismic horizon (light blue) (Figs. 4, 5 and 6). Seismic well ties indicate that the S3 sequence corresponds to the lower section of Montera Formation, and ranges in age within lower Middle Miocene ( $15.9-14$ Ma) (Fig. 4). On the western margin of the basin (Fig. 7) and on the flanks of intrabasinal highs the S3 sequence onlaps the SB1 seismic horizon (Figs. 12D - F) whereas on the southwestern depocentre margins it is truncated against faults or pre-existing topography (Fig. 6D). The SB4 is relatively continuous and composed of a moderate to strong peak that expands conformably and locally truncates S3 reflections. 


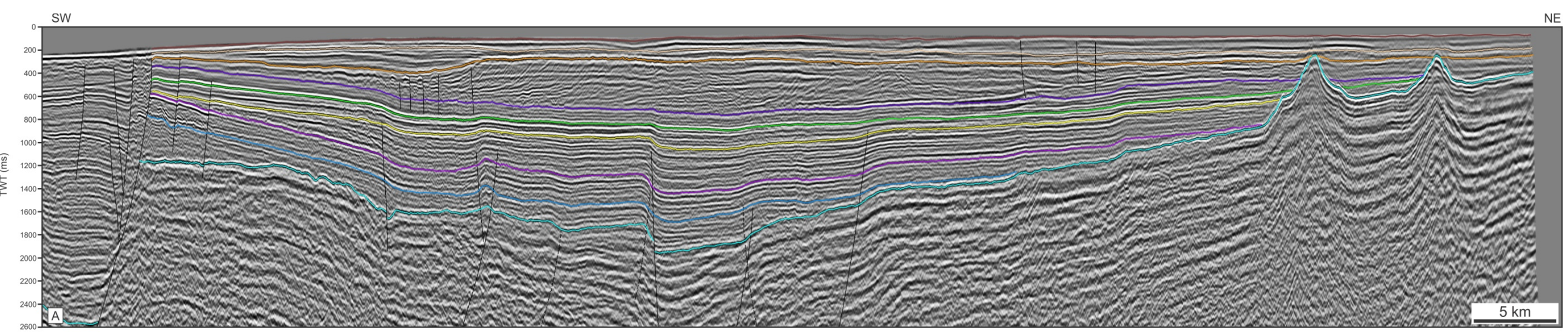

\begin{tabular}{|c|c|}
\hline $\begin{array}{l}\text { Seismic stratigraphic } \\
\text { sequences }\end{array}$ & Series \\
\hline $\begin{array}{l}\text { sit } \\
\text { s10 } \\
59\end{array}$ & Pleistocene \\
\hline S8 & \multirow{2}{*}{ Pliocene } \\
\hline S7 & \\
\hline S6 & \multirow[b]{2}{*}{$\begin{array}{c}\text { Upper } \\
\text { Miocene }\end{array}$} \\
\hline S5 & \\
\hline S4 & \multirow{2}{*}{$\begin{array}{c}\text { Middle } \\
\text { Miocene }\end{array}$} \\
\hline S3 & \\
\hline S2 & $\begin{array}{l}\text { Lower } \\
\text { Miocene }\end{array}$ \\
\hline S1 & $\begin{array}{l}\text { Up.9.9 } \\
\text { Upocene }\end{array}$ \\
\hline
\end{tabular}
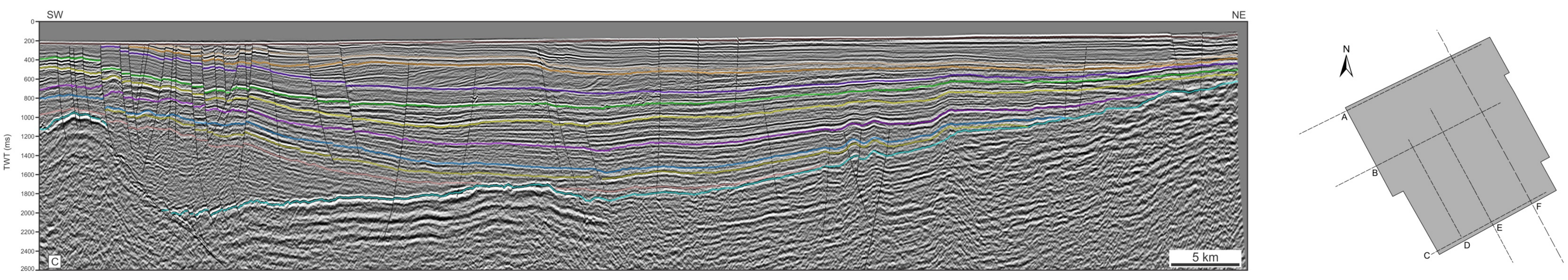

Figure 5. NE-SW Seismic sections of Salaverry Basin with interpreted Cenozoic seismic sequences S1 to S11. Seismic section locations in Figure 3. 

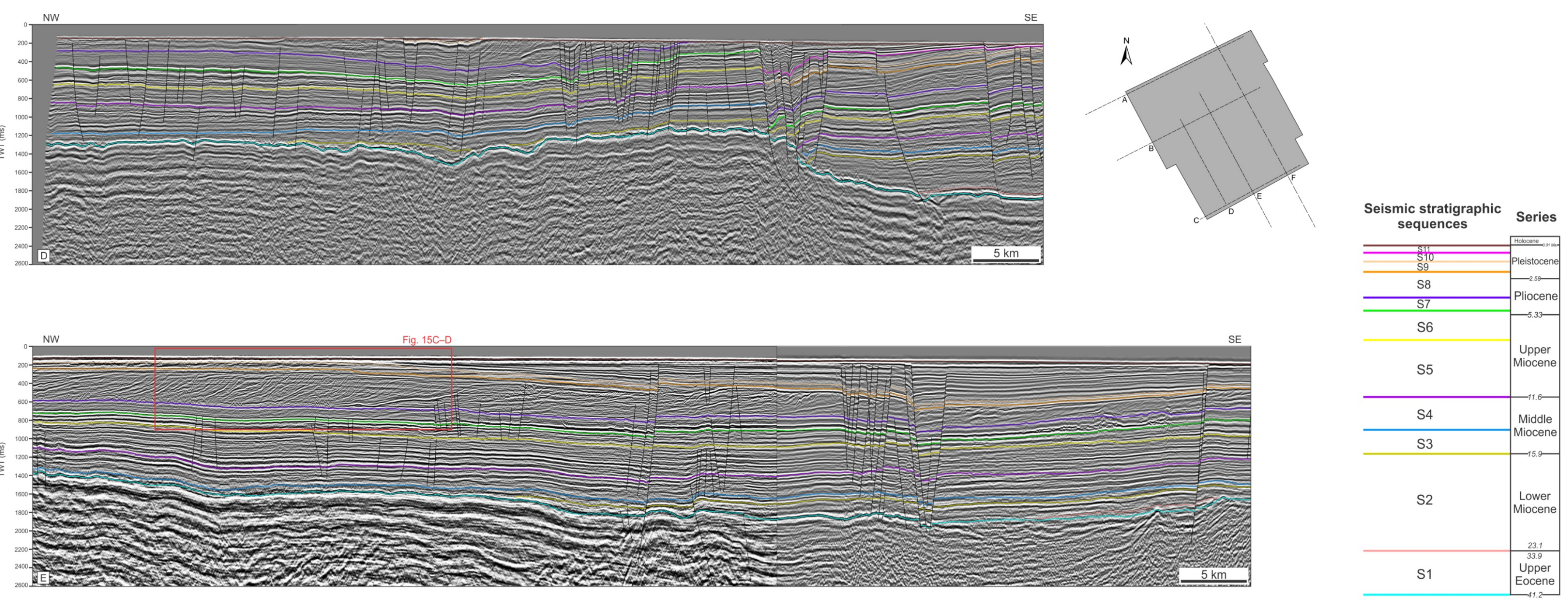

Top of Pre-Cenozoic Basement

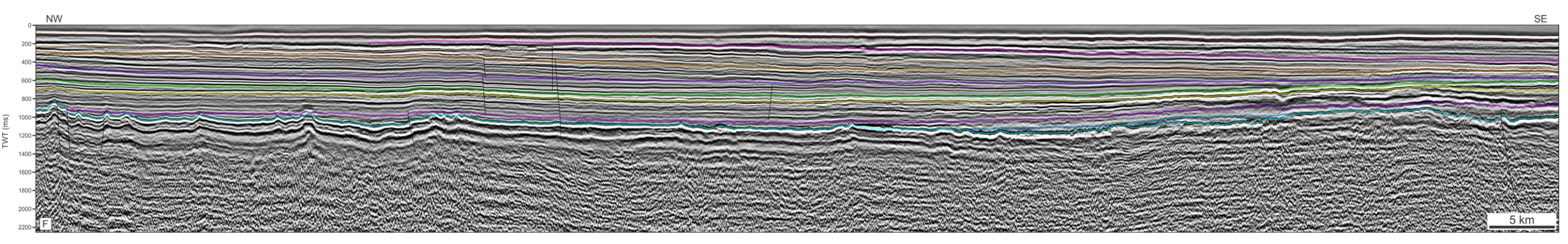

Figure 6. NW-SW Seismic sections of Salaverry Basin with interpreted Cenozoic seismic sequences S1 to S11. Seismic section locations in Figure 3. 
The S3 Isochron map shows increase in thickness mainly to the west $(150-250$ ms TWT), to the north (120 - $170 \mathrm{~ms}$ TWT), and minor part of southwestern depocentre $(120-150 \mathrm{~ms}$ TWT). S3 thins towards the eastern margin of the basin, and in contrast to the $\mathrm{S} 1$ and $\mathrm{S} 2$ sequences, it is relatively thin in the southwestern depocentre $(20-50$ ms TWT) (Fig. 8). Internally, the S3 sequence is dominated by SF6 and SF2 seismic facies. Both seismic facies are organized in sheet-like geometry draping the pre-existing topography. On the eastern margin, S3 is predominantly condensed and westward passes transitionally to SF6 and then to SF2. In addition, on the western margin, sheet and sheet drape bodies of SF10, SF3, and SF4 reflections patch the area. Also chaotic/contorted seismic facies (SF11) are observed westward (Fig. 10, Table 1).

\section{Seismic sequence $\mathrm{S} 4$}

The S4 third-order sequence is bounded at its base and top by SB4 (light blue) and SB5 (purple) seismic horizons, respectively (Figs. 4, 5 and 6). Likewise, towards the eastern margin of the basin SB4 is overstepped by SB1, which also bounding the base of S4 sequence (Fig. 13). Litostratigraphically, it comprises the upper section of Montera Formation and ranges in age within upper Middle Miocene $(\sim 14-11.6 \mathrm{Ma})$ (Fig. 4). The S4 sequence onlaps the SB1 seismic horizon over the eastern margin, conformably overlies the deepest parts of the basin, and truncates against SB5 seismic horizon over the western margin (Figs. 7 and 13). The SB5 seismic horizon is a continuous and moderate peak that corresponds to a local unconformity over the western margin based on biostratigraphic, well log and seismic data (Figs. 4 and 7).

The S4 is thickest $(300-410 \mathrm{~ms}$ TWT) in a wider area of the main basin compared to the S3 sequence. On the eastern margin, the $\mathrm{S} 4$ sequence thins $(10-50 \mathrm{~ms}$ TWT) whereas it is influenced by faulting towards the southwestern depocentre margins (Figs. 6D and 8). The bulk of S4 seismic sequence consists of SF4 seismic facies that spans all over the Cenozoic Salaverry Basin. Eastward the S4 is condensed along a narrow and continuous NW-SE fringe. This condensed portion of S4 passes laterally to SF6, and then to SF2 reflections. On the southwestern depocentre margins, the seismic facies are unclear due to seismic quality decrease by faulting influence. Westward chaotic/contorted (SF11) and associated sigmoid/oblique (SF8) seismic facies that progradate to the west are recognized. The contact between SF11 and SF2 seismic facies is generally abrupt (Fig. 10, Table 1). 
Table 1

Seismic Facies identified in the Cenozoic succession of Salaverry Basin.

\begin{tabular}{|c|c|c|c|c|c|c|c|}
\hline Seismic Facies & $\begin{array}{l}\text { Reflection } \\
\text { configuration }\end{array}$ & $\begin{array}{l}\text { Reflection } \\
\text { continuity }\end{array}$ & $\begin{array}{l}\text { Reflection } \\
\text { amplitude }\end{array}$ & $\begin{array}{l}\text { Reflection } \\
\text { frequency }\end{array}$ & $\begin{array}{l}\text { Boundaries } \\
\text { (lower; upper) }\end{array}$ & $\begin{array}{l}\text { External } \\
\text { form }\end{array}$ & $\begin{array}{l}\text { Seismic section example } \\
\text { (scale bar is } 100 \mathrm{~ms} \text { ) }\end{array}$ \\
\hline $\begin{array}{l}\text { SF1: Parallel, high } \\
\text { continuity, high } \\
\text { amplitude }\end{array}$ & Parallel & Continuous & $\begin{array}{l}\text { Predominantly } \\
\text { high amplitude }\end{array}$ & $\begin{array}{l}\text { Intermediate to } \\
\text { low frequency }\end{array}$ & $\begin{array}{l}\text { Concordant; } \\
\text { Concordant }\end{array}$ & $\begin{array}{l}\text { Sheet, sheet } \\
\text { drape }\end{array}$ & సN= \\
\hline $\begin{array}{l}\text { SF3: Parallel, high } \\
\text { continuity, low } \\
\text { amplitude }\end{array}$ & $\begin{array}{l}\text { Parallel to } \\
\text { subparallel }\end{array}$ & $\begin{array}{l}\text { Relatively } \\
\text { continuous }\end{array}$ & $\begin{array}{l}\text { Predominantly } \\
\text { low amplitude }\end{array}$ & $\begin{array}{l}\text { Intermediate to } \\
\text { low frequency }\end{array}$ & $\begin{array}{l}\text { Concordant, onlap; } \\
\text { Concordant }\end{array}$ & $\begin{array}{l}\text { Sheet drape, } \\
\text { sheet, wedge }\end{array}$ & 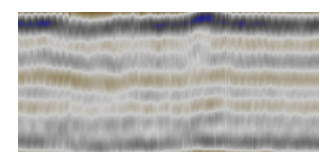 \\
\hline $\begin{array}{l}\text { SF4: Parallel, continuos } \\
\text { and disrupted in part, low } \\
\text { to high amplitude }\end{array}$ & $\begin{array}{l}\text { Parallel to } \\
\text { subparallel }\end{array}$ & $\begin{array}{l}\text { Continuous and } \\
\text { disrupted/broken } \\
\text { in part }\end{array}$ & $\begin{array}{l}\text { Moderate to high } \\
\text { amplitude }\end{array}$ & $\begin{array}{l}\text { Intermediate to } \\
\text { low frequency }\end{array}$ & $\begin{array}{l}\text { Baselap, concordant; } \\
\text { concordant }\end{array}$ & $\begin{array}{l}\text { Sheet drape, } \\
\text { sheet }\end{array}$ & NNST \\
\hline $\begin{array}{l}\text { SF5: Parallel, continuous } \\
\text { and disrupted in part, low } \\
\text { to very low amplitude }\end{array}$ & $\begin{array}{l}\text { Parallel to } \\
\text { subparallel }\end{array}$ & $\begin{array}{l}\text { Continuous and } \\
\text { disrupted/broken } \\
\text { in part }\end{array}$ & $\begin{array}{l}\text { Predominantly } \\
\text { low to very low } \\
\text { amplitude }\end{array}$ & $\begin{array}{l}\text { Intermediate to } \\
\text { low frequency }\end{array}$ & $\begin{array}{l}\text { Concordant; } \\
\text { Concordant }\end{array}$ & $\begin{array}{l}\text { Sheet drape, } \\
\text { sheet }\end{array}$ & 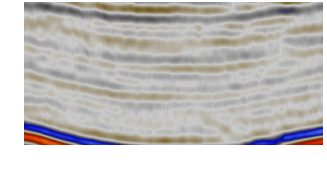 \\
\hline $\begin{array}{l}\text { SF6: Subparallel, low } \\
\text { continuity, variable } \\
\text { amplitude }\end{array}$ & Subparallel & Discontinuos & $\begin{array}{l}\text { Predominantly } \\
\text { low, but variable } \\
\text { amplitude }\end{array}$ & $\begin{array}{l}\text { Intermediate to } \\
\text { low frequency }\end{array}$ & $\begin{array}{l}\text { Baselap, concordant; } \\
\text { Concordant, } \\
\text { truncation }\end{array}$ & $\begin{array}{l}\text { Wedge, } \\
\text { sheet drape }\end{array}$ & 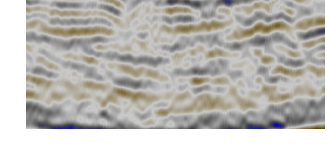 \\
\hline
\end{tabular}


Table 1

Seismic Facies identified in the Cenozoic succession of Salaverry Basin.

\begin{tabular}{|c|c|c|c|c|c|c|c|}
\hline Seismic Facies & $\begin{array}{l}\begin{array}{l}\text { Reflection } \\
\text { configuration }\end{array} \\
\end{array}$ & $\begin{array}{l}\text { Reflection } \\
\text { continuity }\end{array}$ & $\begin{array}{l}\text { Reflection } \\
\text { amplitude }\end{array}$ & $\begin{array}{l}\text { Reflection } \\
\text { frequency }\end{array}$ & $\begin{array}{l}\begin{array}{l}\text { Boundaries } \\
\text { (lower; upper) }\end{array} \\
\end{array}$ & $\begin{array}{l}\text { External } \\
\text { form }\end{array}$ & $\begin{array}{c}\text { Seismic section example } \\
\text { (scale bar is } 100 \mathrm{~ms} \text { ) }\end{array}$ \\
\hline $\begin{array}{l}\text { SF7: Divergent, low to } \\
\text { high amplitude }\end{array}$ & Divergent & $\begin{array}{l}\text { Relatively } \\
\text { continuous }\end{array}$ & $\begin{array}{l}\text { Predominantly low } \\
\text { but also high } \\
\text { amplitude }\end{array}$ & $\begin{array}{l}\text { Intermediate to } \\
\text { low frequency }\end{array}$ & $\begin{array}{l}\text { Baselap, concordant; } \\
\text { Truncation, } \\
\text { concordant }\end{array}$ & Wedge & \\
\hline $\begin{array}{l}\text { S F8: Sigmoid/oblique } \\
\text { amplitudes }\end{array}$ & $\begin{array}{l}\text { Prograding } \\
\text { clinoform }\end{array}$ & $\begin{array}{l}\text { Relatively } \\
\text { continuous, } \\
\text { internal reflectors } \\
\text { are quiet } \\
\text { discontinuous }\end{array}$ & $\begin{array}{l}\text { Low to moderate. } \\
\text { High amplitude } \\
\text { at bottomsets. }\end{array}$ & $\begin{array}{l}\text { Intermediate to } \\
\text { low frequency }\end{array}$ & $\begin{array}{l}\text { Downlap, } \\
\text { concordant; } \\
\text { Toplap, concordant }\end{array}$ & $\begin{array}{l}\text { Elongated lens, } \\
\text { and channel } \\
\text { fill }\end{array}$ & \\
\hline $\begin{array}{l}\text { SF9: Concave upward } \\
\text { amplitudes }\end{array}$ & $\begin{array}{l}\text { Prograding } \\
\text { clinoform }\end{array}$ & $\begin{array}{l}\text { Relatively } \\
\text { continuous, } \\
\text { internal reflectors } \\
\text { are quiet } \\
\text { discontinuous }\end{array}$ & $\begin{array}{l}\text { Low to high } \\
\text { amplitude. } \\
\text { Bursts of high } \\
\text { amplitude at foresets } \\
\text { and bottomsets. }\end{array}$ & $\begin{array}{l}\text { Intermediate to } \\
\text { high frequency }\end{array}$ & $\begin{array}{l}\text { Downlap, } \\
\text { concordant; } \\
\text { Toplap, concordant }\end{array}$ & Fan & \\
\hline $\begin{array}{l}\text { SF10: Hummocky } \\
\text { amplitudes }\end{array}$ & $\begin{array}{l}\text { Prograding } \\
\text { clinoform }\end{array}$ & $\begin{array}{l}\text { Irregular } \\
\text { discontinuous }\end{array}$ & $\begin{array}{l}\text { Predominantly } \\
\text { low to moderate. }\end{array}$ & $\begin{array}{l}\text { Intermediate to } \\
\text { low frequency }\end{array}$ & $\begin{array}{l}\text { Concordant, baselap; } \\
\text { Concordant }\end{array}$ & $\begin{array}{l}\text { Sheet drape, } \\
\text { sheet, wedge }\end{array}$ & \\
\hline $\begin{array}{l}\text { SF1 1: Chaotic and } \\
\text { contorted amplitudes }\end{array}$ & $\begin{array}{l}\text { Chaotic, } \\
\text { contorted }\end{array}$ & $\begin{array}{l}\text { Very discontinuous. } \\
\text { Short segments } \\
\text { in contorted and } \\
\text { low subparallel to } \\
\text { wavy patterns }\end{array}$ & $\begin{array}{l}\text { Low to high } \\
\text { amplitude }\end{array}$ & $\begin{array}{l}\text { Intermediate to } \\
\text { low frequency }\end{array}$ & $\begin{array}{l}\text { Few individual } \\
\text { segments are onlap, } \\
\text { concordant; } \\
\text { Concordant }\end{array}$ & $\begin{array}{l}\text { Slope front } \\
\text { fill, } \\
\text { channel fill }\end{array}$ & 5 \\
\hline $\begin{array}{l}\text { SF12: Mounded } \\
\text { amplitudes }\end{array}$ & Mounded & $\begin{array}{l}\text { Relatively } \\
\text { discontinuous }\end{array}$ & $\begin{array}{l}\text { Low to high } \\
\text { amplitude. Bursts } \\
\text { of high amplitude } \\
\text { at the base. }\end{array}$ & $\begin{array}{l}\text { Intermediate to } \\
\text { low frequency }\end{array}$ & $\begin{array}{l}\text { Concordant, few } \\
\text { individual segments } \\
\text { are onlap; } \\
\text { Concordant }\end{array}$ & Mound & ans \\
\hline
\end{tabular}




\section{Seismic Sequence S5}

The S5 second-order sequence is bounded at its base by SB5 seismic horizon (purple) and at its top by SB6 seismic horizon (yellow) (Figs. 4, 5 and 6). Similar to S4 sequence towards the eastern margin of the basin SB5 is overstepped by SB1, and thus also bound the base of S5 sequence (Fig. 13). Well calibration shows that S5 corresponds to lower-middle section of Zapallal Formation, and ranges in age within the Upper Miocene ( 11.6 - $7 \mathrm{Ma}$ ) (Fig. 4). The SB6 is a moderate to strong peak with consistent lateral continuity. This horizon is a seismic unconformity marked by local truncation below and baselap above (Figs. 7 and 13) whereas is conformable over the eastern margin and the deepest parts of the basin (Figs. 5C, 6D and 13).
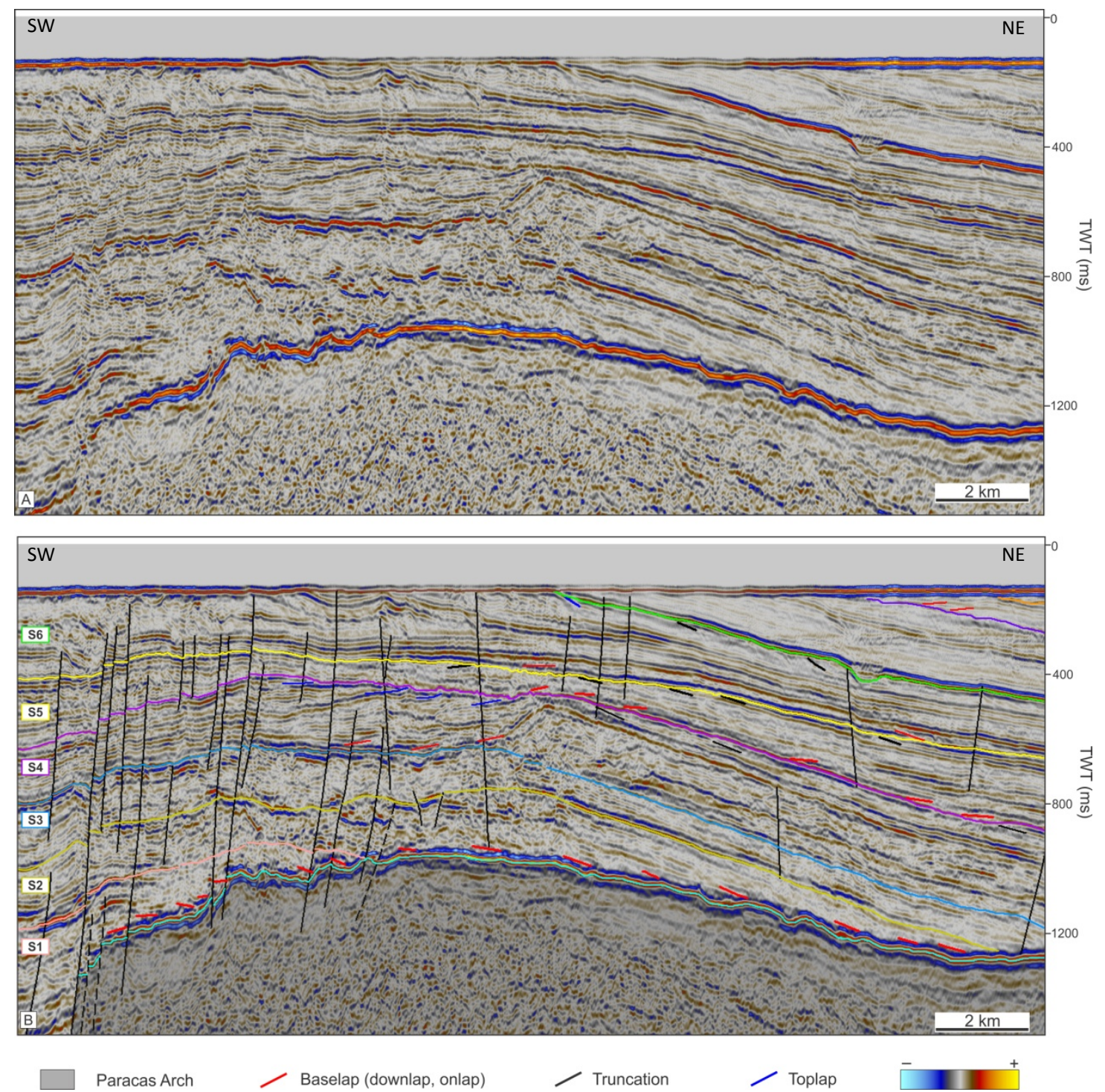

Figure 7. Reflection configuration and terminations within the Cenozoic seismic sequences. A) Uninterpreted and B) interpreted 2D seismic section across the western margin of the basin over the Paracas Arch. The location of the seismic section is indicated in Figure 5B. 
The S5 Isochron map shows the thickest sedimentary fill along the basin foredeep all over the study area $(300-420 \mathrm{~ms}$ TWT). The S5 sequence thins towards the eastern $(<30 \mathrm{~ms}$ TWT) and western $(<50 \mathrm{~ms}$ TWT) margins of the basin; and is absent over the volcanic arc and Coastal Batholith domain (Figs. 8 and 14).

The seismic signature of S5 in the main basin consists of SF4 and SF2 seismic facies. Along the eastern margin, SF6 reflections are organized in wedge and sheet drape bodies that conforms a continuous NW- SE fringe similar to S4 sequence. On the western margin, the S5 sequence is characterized by divergent seismic facies (SF7). In addition, on the southwestern depocentre margins the seismic quality is poor due to faulting, and therefore the seismic facies are unclear (Fig. 10, Table 1).

\section{Seismic sequence S6}

The S6 third-order sequence is bounded at its base and top by SB6 seismic horizon (yellow) and SB7 seismic horizon (green), respectively (Figs. 4, 5 and 6). It comprises the upper section of Zapallal Formation and the basal section of the Miramar Formation, ranging in age from Upper Miocene to Lower Pliocene ( $7-5 \mathrm{Ma})$ (Fig. 4). The S6 downlaps the SB6 over the western margin (Fig. 7), is conformable in the main basin, and baselaps the SB6 over the eastern margin (Fig. 13). The SB7 seismic horizon is a continuous and strong peak marked by local truncation below that evidences a seismic unconformity over the western margin (Fig. 7).

The S6 geographic extent corresponds to almost all over the study area and only is absent over the volcanic arc and Coastal Batholith domain, near to the coastline. The S6 sequence is thickest over the western margin, where it reaches up to $250-300 \mathrm{~ms}$ TWT, and is consistently thin $(<30 \mathrm{~ms}$ TWT) along a $12 \mathrm{~km}$ wide continuous fringe on the eastern margin (Fig. 9). The S6 seismic sequence is very similar to S5 in seismic facies signature and distribution (Figs. 10 and 11). Cenozoic Salaverry Basin is dominated by SF4 reflections. On the eastern margin, the S6 is locally condensed and passes transitionally to SF6 seismic facies. Some minor sheet drape bodies of SF2 and patches of SF1 seismic facies are recognized towards the north and west. In addition on the western margin, divergent seismic facies (SF7) pass abruptly to chaotic/contorted seismic facies (SF11) (Fig. 11, Table 1). 

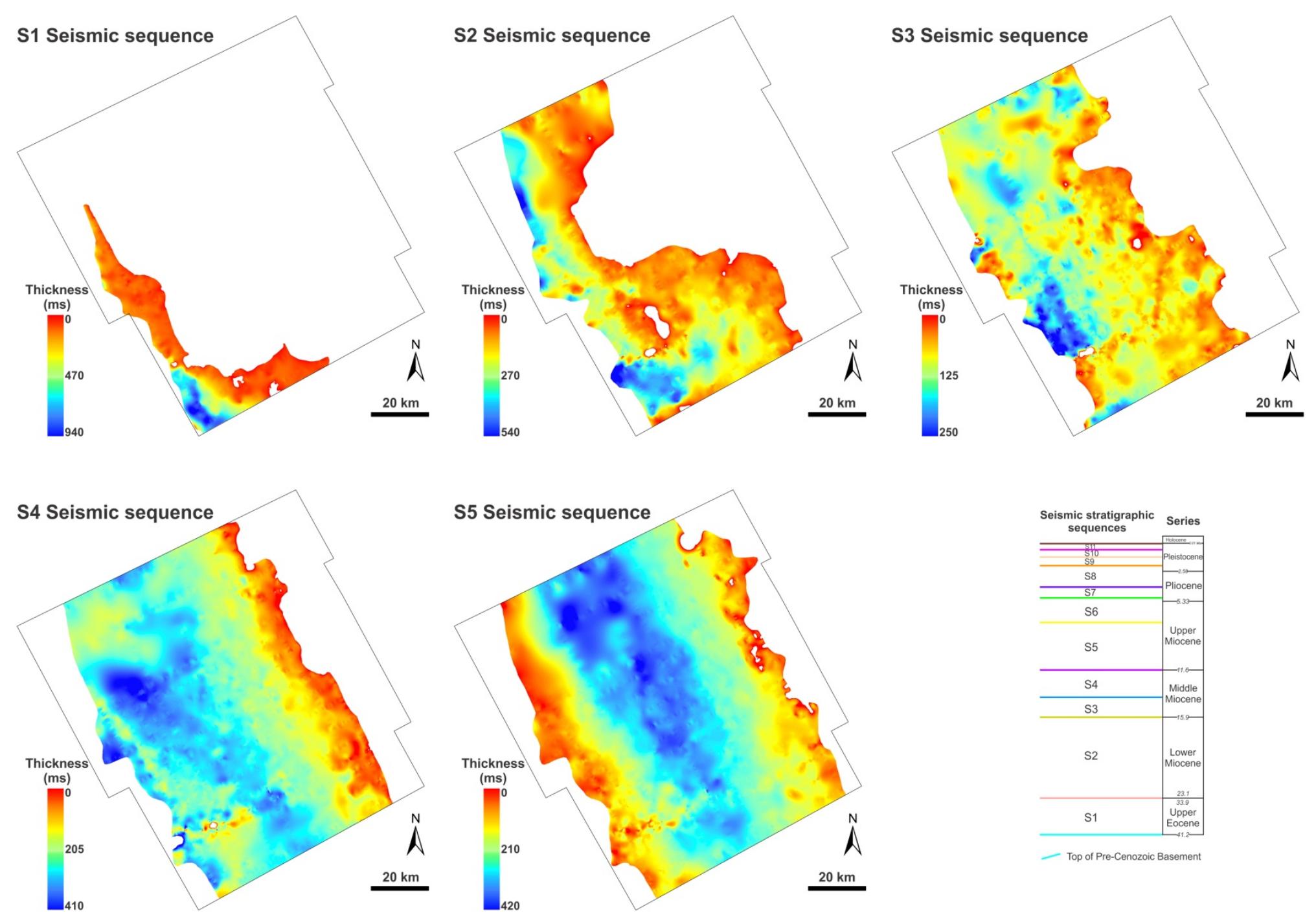

Figure 8. Isochron maps of the seismic stratigraphic sequences S1 to S5. Isochron maps depict the two way time (TWT) thickness calculated between top and bottom sequence boundary of each seismic stratigraphic sequence. White areas within the study area (black polygon) depict absence of the sequence or lack seismic interpretation due to data gaps or poor seismic quality. 

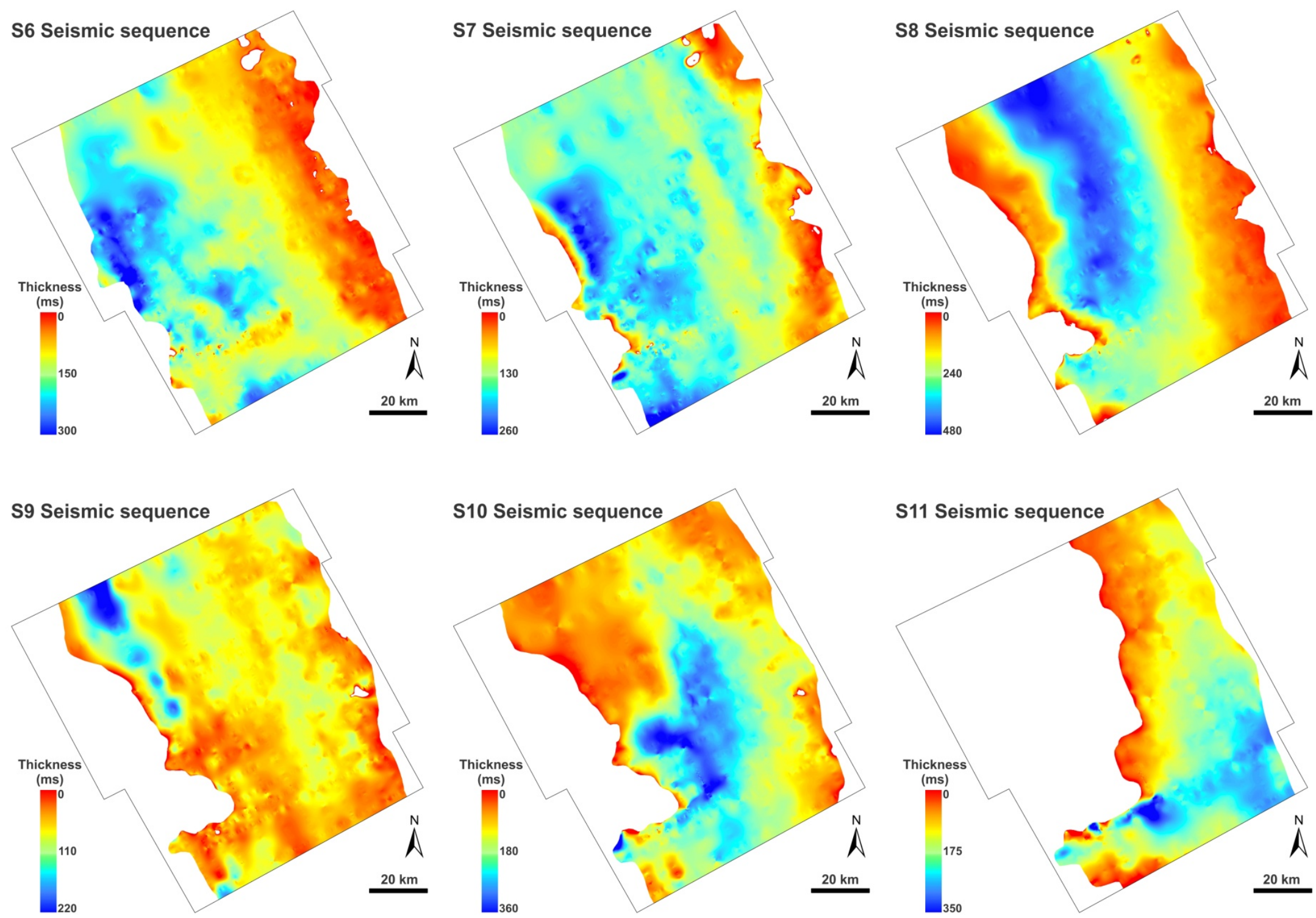

Figure 9. Isochron maps of the seismic stratigraphic sequences S6 to S11. 


\section{Seismic sequence $\mathbf{S 7}$}

The S7 third-order sequence is bounded at its base by SB7 seismic horizon (green) and at its top by SB8 seismic horizon (blue) (Figs. 4, 5 and 6). S7 has not been drilled yet in the basin, thus based on seismic stratigraphic interpretation it would comprises the middle section of Miramar Formation, ranging in age within the Lower Pliocene ( 5-4 Ma) (Fig. 4). The S7 predominantly rest conformable draping the preexisting topography and only is truncated against volcanic/intrusive bodies that act as shallow morphostructural highs, on the eastern margin of the basin (Fig. 14). The SB8 is a moderate to strong peak marked by regional downlap above and local toplap below that evidence a seismic unconformity all over the study area (Figs. 7 and 13). Wide geographic extent characterize the S7 sequence, which only is absent over the volcanic arc and Coastal Batholith domain. The S7 Isochron map shows major thickness in the central part of western margin (200 - $240 \mathrm{~ms}$ TWT) and in the southwestern depocentre (230 - 260 ms TWT) (Fig. 9).

The bulk of S7 sequence is dominated by three seismic facies consistently all over the study area: SF5 span over the western margin; SF4 extend along the basin foredeep; and SF6 characterize the eastern margin (Fig. 11, Table 1). For the first time in the study area, mounded seismic facies (SF12) are recognized as patches $(\sim 10-30 \mathrm{~km}$ wide) in the central part of the basin (Fig. 11, Table 1). In addition, sheet and sheet drape bodies of SF2 reflections patch considerable areas in the basin. On the other hand, the S7 seismic sequence is locally condensed eastward.

\section{Seismic sequence $\mathrm{S8}$}

The S8 third-order sequence is bounded at its base and top by SB8 seismic horizon (blue) and SB9 seismic horizon (orange), respectively (Figs. 4, 5 and 6). Analogous to S7 sequence, it has not been drilled in the basin but seismic interpretation suggests that correlates to the upper section of Miramar Formation and the basal section of Hornillos Formation, ranging in age from Upper Pliocene to Lower Pleistocene ( $\sim-2 \mathrm{Ma})$ (Fig. 4). The S8 consistently downlaps the SB8 over the study area, where the prograding clinoforms have influence (Fig. 11 and 15). The SB9 seismic horizon is a moderate peak with consistent lateral continuity all over the study area. Likewise, it is marked by toplap and concordant reflections below and baselap (downlap, onlap) above (Fig. 15). 

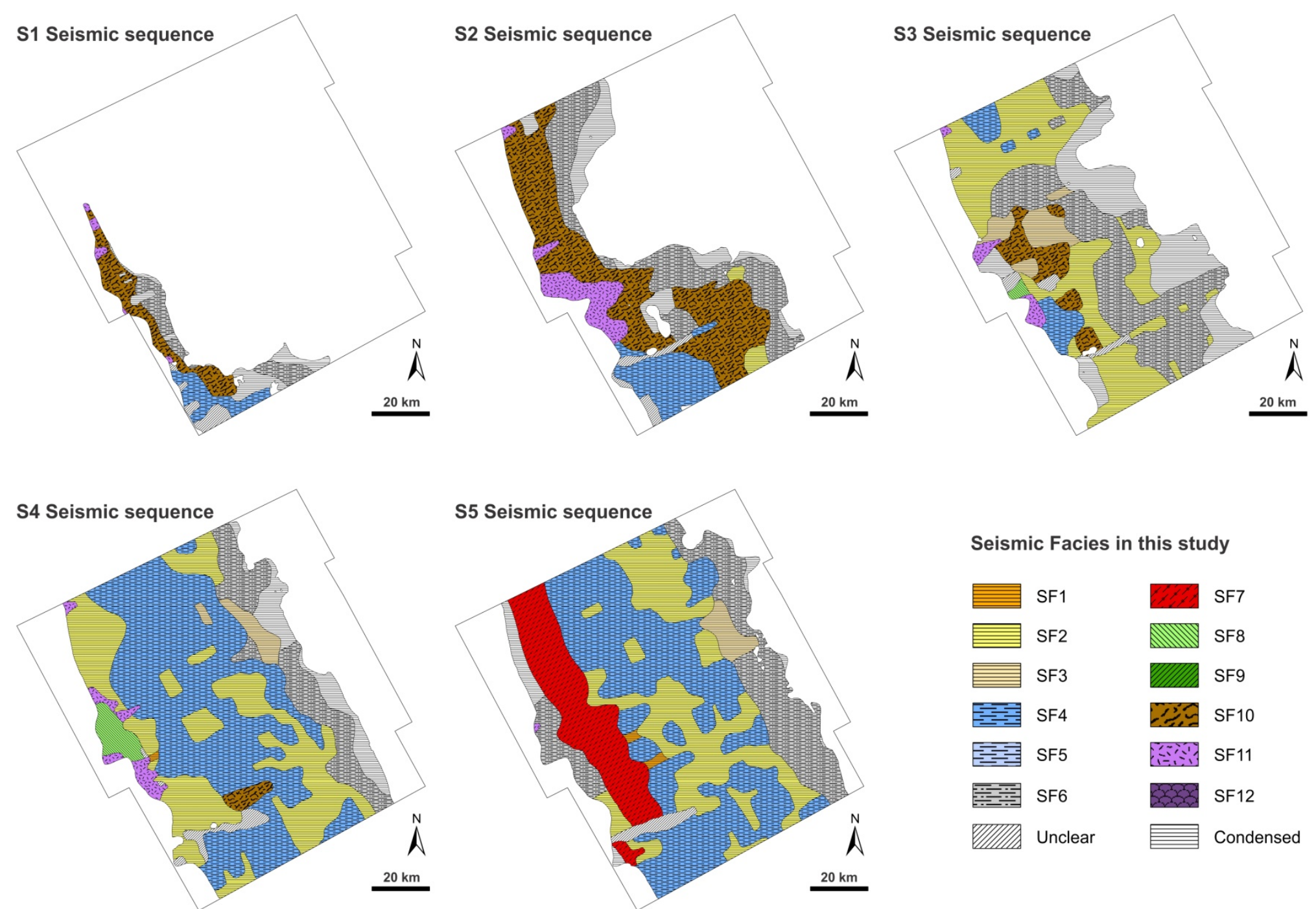

Seismic Facies in this study

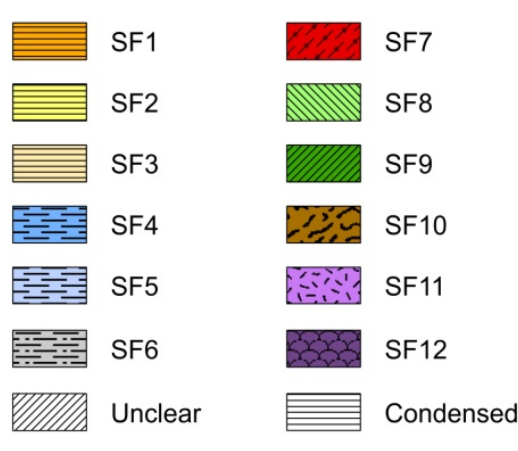

Figure 10. Seismic facies maps of the seismic stratigraphic sequences S1 to S5. Number of seismic facies in the study area (black polygon) increases from the S1 to the S11 sequences indicating that different depositional processes occurred during their deposition. Refer to Figure 3 for position of the basin and structural highs. See text for further explanation. 

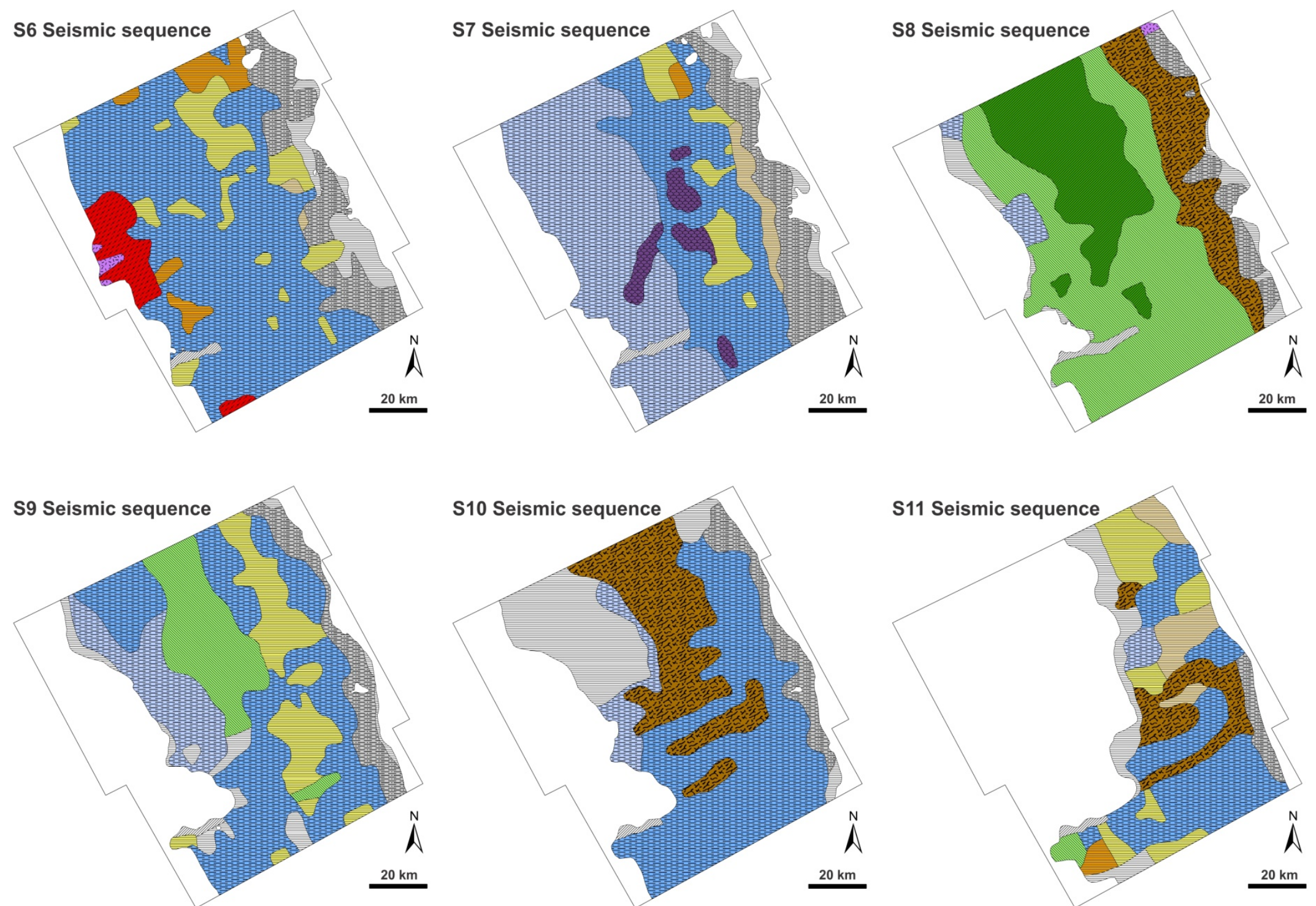

Figure 11. Seismic facies maps of the seismic stratigraphic sequences S6 to S11. See Table 1 for seismic facies description. 
S8 geographic extent is very similar to S7 and S6. A major delta that progradates from SE to NW is recognized in regional seismic sections (Figs. 5A, 5B and 15). The S8 sequence reaches maximum thickness $(350-480 \mathrm{~ms}$ TWT $)$ in the central part of this delta and thins $(200-250 \mathrm{~ms}$ TWT $)$ towards its flanks. On the eastern margin the S8 sequence is very thin $(<50 \mathrm{~ms}$ TWT $)$ to absent, near to the coastline; and analogous behavior is observed on the western margin ( $<30 \mathrm{~ms}$ TWT) (Fig. 9).

Internally, the seismic signature of S8 sequence in the study area is characterized by different types of prograding clinoforms: sigmoid/oblique (SF8), concave upward (SF9), and hummocky (SF10) seismic facies. Spatial-geometric relationships between these seismic facies evidence at least three major progradational events in the study area that would be studied in a further work (Fig. 11 and Table 1).

\section{Seismic sequence $\mathbf{S 9}$}

The S9 third-order sequence is bounded at its base by SB9 seismic horizon (orange) and at its top by SB10 seismic horizon (peach orange) (Figs. 4, 5 and 6). It has not been drilled in the basin but seismic interpretation suggests that correlates to the lower section of Hornillos Formation, and ranges in age within the Lower Pleistocene $(\sim 2-1.4 \mathrm{Ma})$ (Fig. 4).

The S9 predominantly rest conformable draping the pre-existing topography (Fig. 13) and locally baselaps on the SB9 towards the western margin. The SB10 is a continuous and moderate peak marked by toplap and truncation below and baselap (downlap, onlap) above that evidence a seismic unconformity all over the study area (Figs. 13 and 15).

The S9 sequence has considerable geographic extension, compared to younger sequences, and only is absent on the western margin. The S9 Isochron map shows major thickness in the northeastern part of the study area $(180-220 \mathrm{~ms}$ TWT) and in the south part of the southwestern depocentre (120 - 140 ms TWT) (Fig. 9).

The bulk of S9 sequence consists of SF4, SF2 and SF8 seismic facies. Eastward it is locally condensed and passes transitionally to SF6 reflections. Also SF5 seismic facies are recognized to the west (Fig. 11, Table 1). 

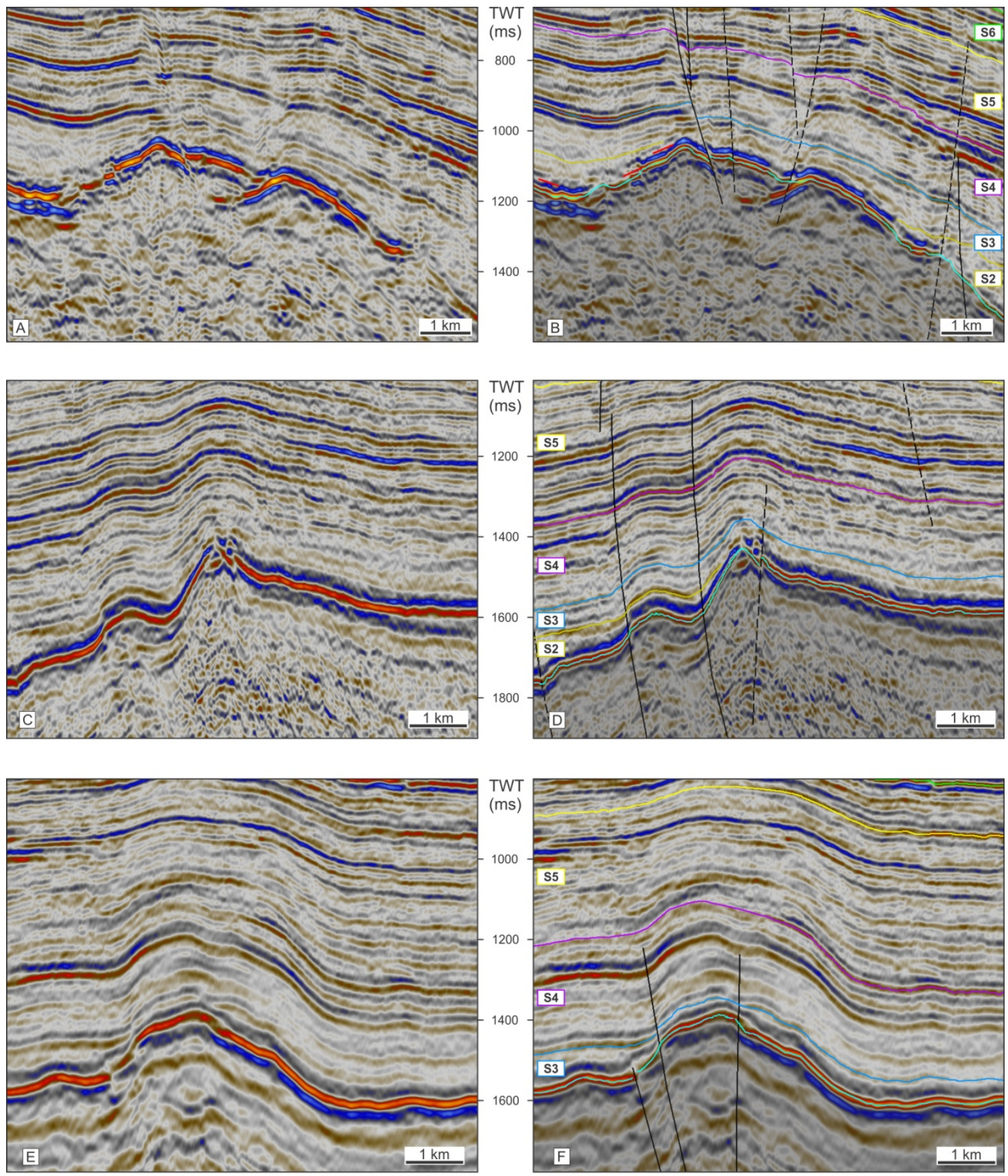

Pre-Cenozoic Basement

Baselap (downlap, onlap)

Truncation $>$ Toplap

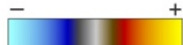

Figure 12. A) Uninterpreted and B) interpreted NE-SW seismic profile across the southern Paracas Arch. Note the thinning and absence of the S2 seismic sequence on the flanks and top of the structural high, respectively. C) Uninterpreted and D) interpreted NE-SW seismic profile across an intrabasinal high, where S3 sequence onlaps the SB1 seismic horizon. E) Uninterpreted and F) interpreted NE-SW seismic profile across the basin foredeep. Note the thinning and onlapping of S3 sequence towards the intrabasinal high flanks. The location of the seismic profiles is indicated in Figure 3. 


\section{Seismic sequence S10}

The S10 third-order sequence is bounded at its base and top by SB10 seismic horizon (peach orange) and SB11 seismic horizon (magenta) (Figs. 4, 5 and 6). Analogous to S9 sequence, it has not been drilled in the basin but seismic interpretation suggests that correlates to the middle section of Hornillos Formation, ranging in age within the Pleistocene ( $1.4-0.6 \mathrm{Ma})$ (Fig. 4). The S10 sequence baselaps consistently on the SB10 in the study area. Likewise, the SB11 is a moderate peak with consistent lateral continuity marked by truncation and concordant reflections below, and regional onlap above that evidence a seismic unconformity (Fig. 13).
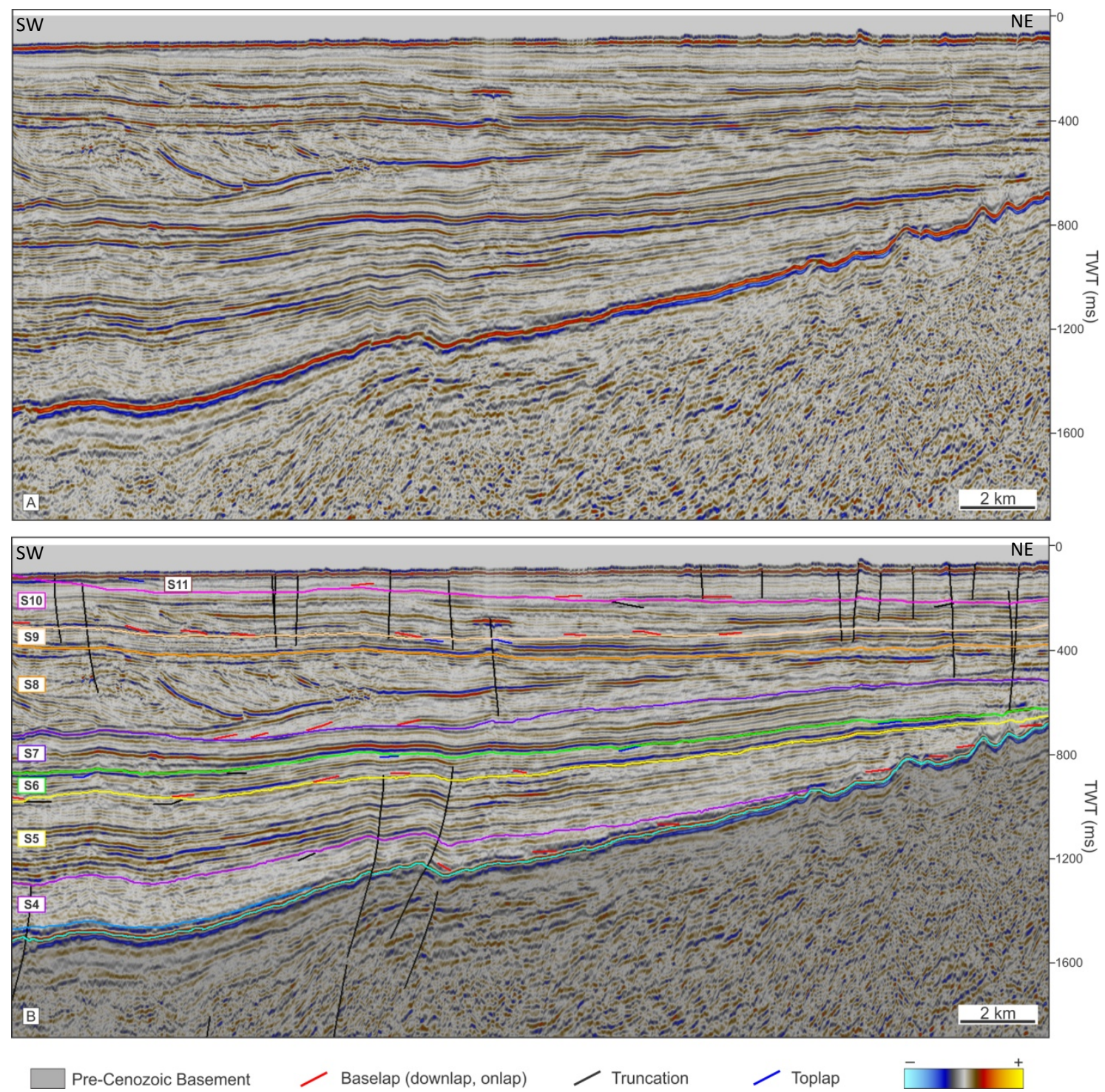

Figure 13. Reflection configuration and terminations within the Cenozoic seismic sequences. A) Uninterpreted and B) interpreted 2D seismic section across the eastern margin of the basin. The location of the seismic section is indicated in Figure 5B. 
The S10 thicks $(200-260$ ms TWT) along the central-south sector of the study area and thins towards the margins $(<40 \mathrm{~ms}$ TWT). Its geographic extent is similar to S9 sequence (Fig. 9). Internally, the S10 sequence is mainly dominated by SF4 and SF10 seismic facies. The S10 is predominantly condensed to the northwestern part of the study area whereas is characterized by SF6 reflections to the eastern margin, near to the coastline. Similar to S9 it is absent on the western margin of the basin (Fig. 11, Table 1).

\section{Seismic sequence S11}

The S11 third-order sequence is bounded at its base by SB11 seismic horizon (magenta) and at its top by SB12 seismic horizon (brown) (Figs. 4, 5 and 6).
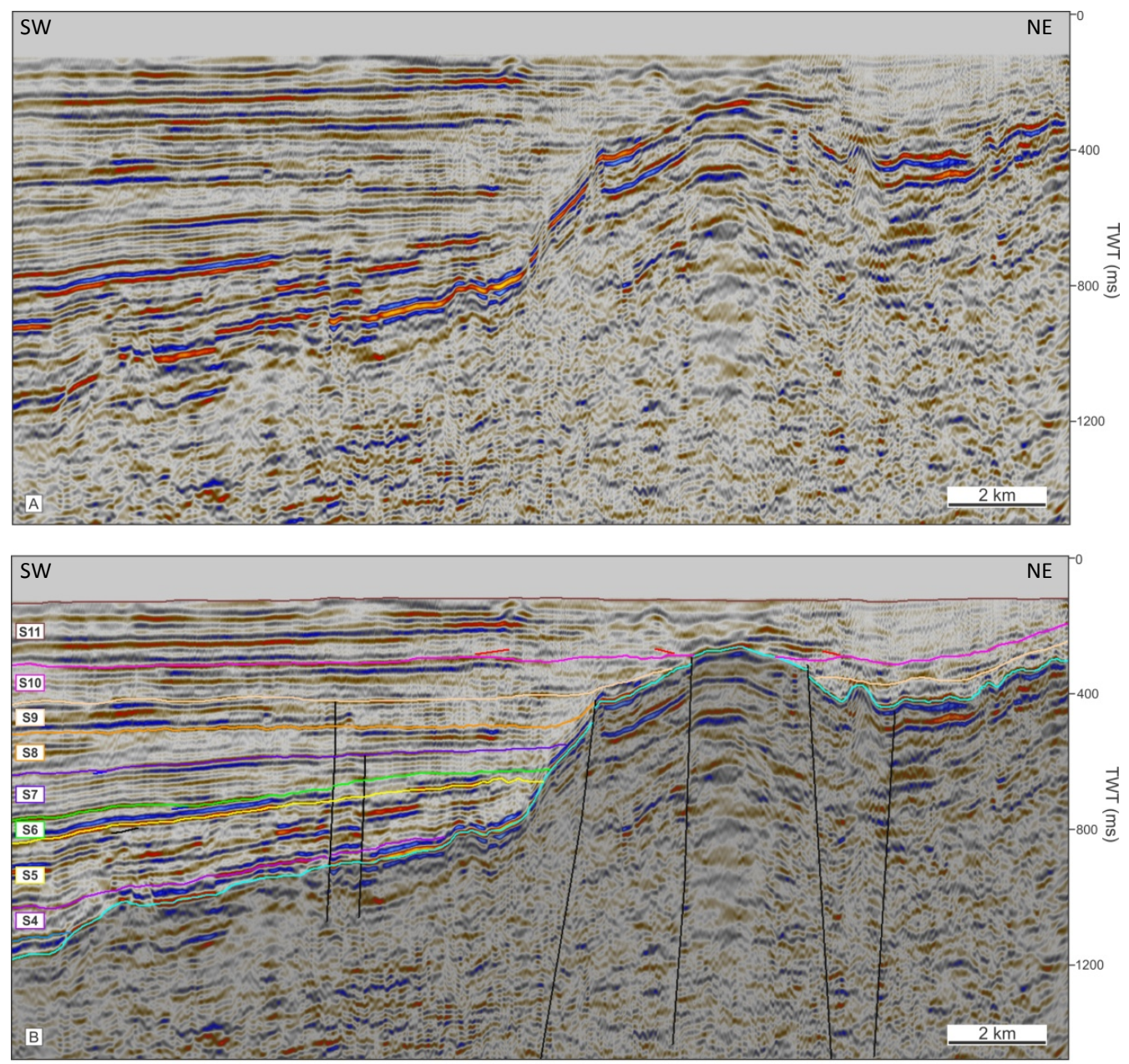

$\square$ Pre-Cenozoic Basement $\quad$ Baselap (downlap, onlap) $\quad$ Truncation $\quad$ Toplap

Figure 14. A) Uninterpreted and B) interpreted NE-SW seismic profile across the eastern margin, showing S4 to S9 sequences truncation against shallow structural high that depicts volcanic/intrusive bodies. The location of the seismic profile is indicated in Figure 3. 
Similar to S7, S8, S9 and S10 it has not been drilled in the basin but seismic interpretation suggests that correlates to the upper section of Hornillos Formation, and ranges in age within Pleistocene ( 0.6-0.01 Ma) (Fig. 4).
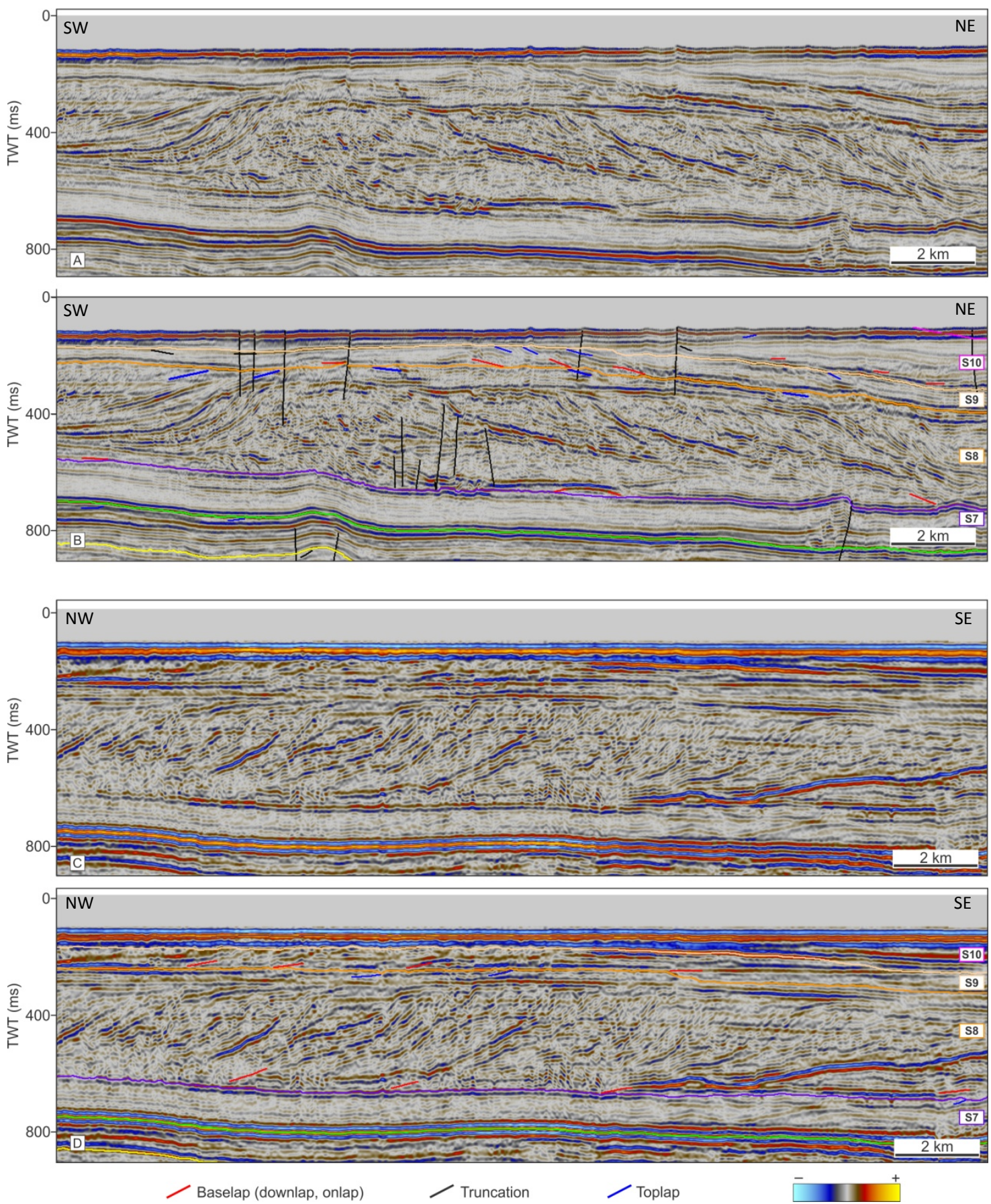

Figure 15. A) Uninterpreted and B) interpreted dip seismic profile across the Cenozoic Salaverry Basin, showing the depositional strike of major delta within the S8 seismic sequence. C) Uninterpreted and D) interpreted strike seismic profile along the basin foredeep, showing the depositional dip of major delta within the S8 seismic sequence. Note the concave upward prograding clinoforms that conform the delta. The location of the seismic profile is indicated in Figures 3 and 6E. 
The S11 sequence baselaps regionally on the SB11. Likewise, the SB12 is a continuous strong peak marked by truncation below and concordant reflections above (Fig. 13). The S11 geographic extent is limited to east and south parts of the study area. The S11 is thickest in the south part of the basin, where it reaches up to $250-350 \mathrm{~ms}$ TWT, whereas is thinnest $(<20 \mathrm{~ms}$ TWT) towards the west (Fig. 9).

The seismic signature of S11 is diverse but mainly is composed by parallel, continuous and disrupted in part, low to high amplitude seismic facies (SF4) and hummocky, low to moderate amplitude reflections (SF10). Several drape and sheet drape bodies patch the study area randomly. These bodies are characterized by SF2, SF3, SF5 and SF1 seismic facies (Fig. 11, Table 1).

\subsection{RESULTS OF THERMOCHRONOLOGICAL ANALYSIS}

Apatite fission track (AFT) thermochronology was applied in two detrital borehole samples from Ballena-1X exploratory well, drilled by Occidental Petroleum Company in 1971 in a current free contract area. The sampling was accomplished by sandstones and conglomeratic sandstones along the Cenozoic sequence at depth between 195 and $963 \mathrm{~m}$. Seven different sample intervals, located between sequence boundaries, were collected; but only two of them (B1 and B7 samples) have enough apatite grains to perform the fission track analysis (Fig. 4).

The ranges and the means of single-grain AFT ages in both detrital samples are similar (varying from 225 to $25 \mathrm{Ma}$ ), but the fission track central age and the age distributions for each sample show differences. However, the samples analyzed passed the $\chi^{2}$-squared test and have an age-dispersion under $15 \%$, indicating that one age component is present in the obtained single grain age population (Fig. 16).

The B1 sample is a Pliocene-Pleistocene (<5.3 Ma) conglomeratic sandstone and presents an apparent age of $53 \pm 5$ Ma. This sample was collected in depth interval of $207-225 \mathrm{~m}$ at the present day temperature of $38^{\circ} \mathrm{C}$. The mean confined fission track lengths are $10.72 \pm 1.47 \mu \mathrm{m}$ and Dpar are $3.4 \mu \mathrm{m}$ (Table 2).

The B7 sample is an Upper Eocene (41.2 - $33.9 \mathrm{Ma})$ fine-grained quartz sandstone and presents an apparent age of $73 \pm 6$ Ma. This sample was collected in 
depth interval of $945-963 \mathrm{~m}$ at the present day temperature of $50^{\circ} \mathrm{C}$. The mean confined fission track lengths are $11.21 \pm 0.93 \mu \mathrm{m}$ and Dpar are $2.9 \mu \mathrm{m}$ (Table 2).

Table 2 summarizes the results of AFT dating. AFT data of samples from Salaverry Basin show cooling ages older than their depositional ages, indicating that these samples record source cooling; it means pre-depositional denudation history.

In this context and in order to further constrain thermal and denudation history of the Salaverry Basin, time-temperature histories have been modeled for B1 and B7 samples. Good fitting $\mathrm{T}-\mathrm{t}$ paths from the B1 sample show relatively fast cooling from the base to the top of the AFT PAZ $\left(60^{\circ} \mathrm{C}\right)$ at ca. $250-220 \mathrm{Ma}$. Thereafter, the samples were reheated to temperatures of ca $110^{\circ} \mathrm{C}$ at ca. $40 \mathrm{Ma}$ before it cooled to present surface conditions (Fig. 16).

The B7 sample which have an Upper Eocene depositional age $(\sim 38 \pm 4 \mathrm{Ma})$ shows a pre-depositional burial, corresponding to temperature increases of $50-100^{\circ} \mathrm{C}$; as well as very limited post-depositional burial heating, consistent with the fact that it has not been significantly reset (Fig. 16). 

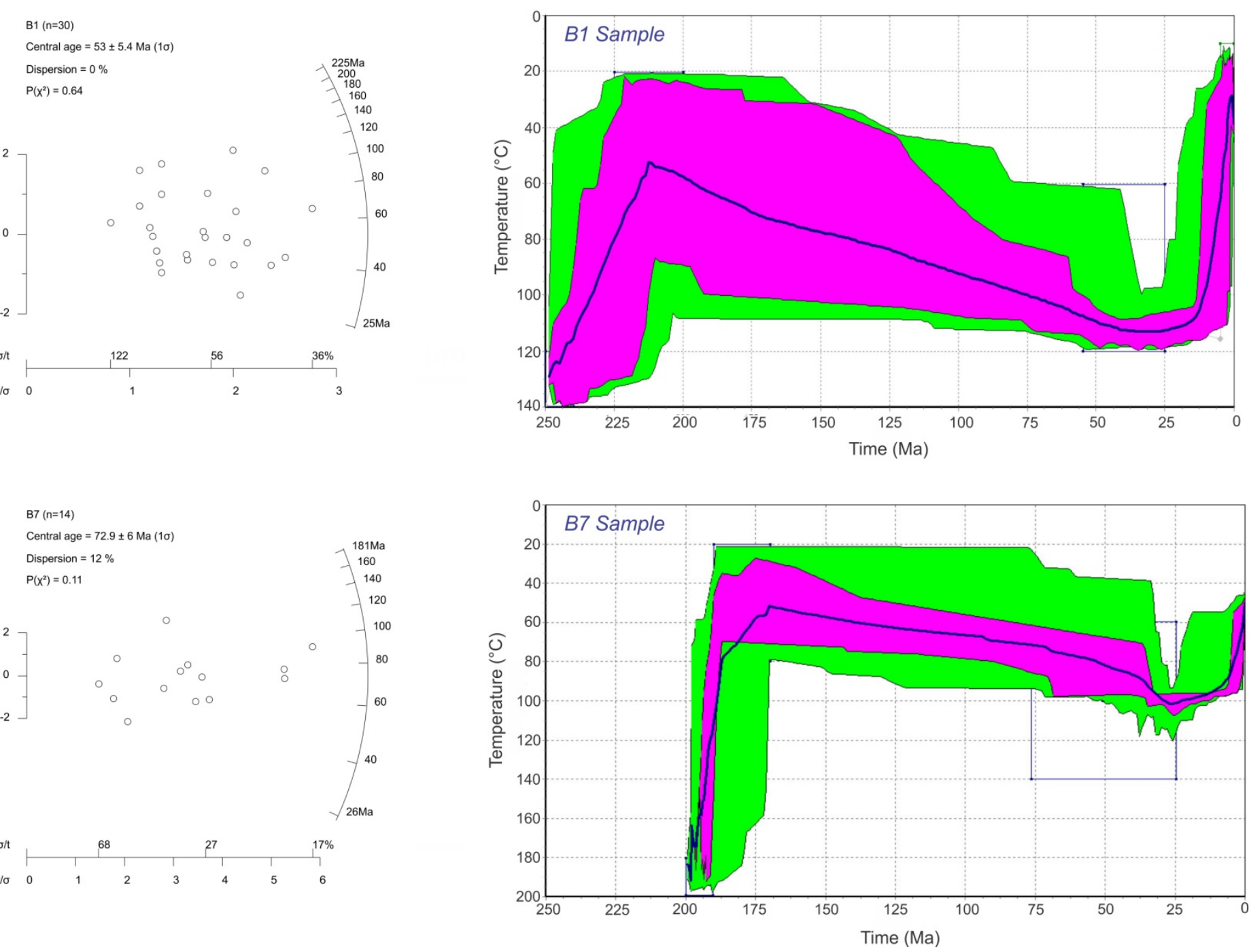

Figure 16. Left column: radial plots showing single grain AFT age in detrital samples B1 and B7. Right column: thermal inverse modeling results of AFT ages from B1 and B7 samples of Ballena-1X exploratory well. The models were obtained with the HeFTy program (Ketcham, 2005) (Blue line: average of the good-fit thermal history. Magenta area: statistically good fit paths. Green area: acceptable fit paths). 
Table 2

Apatite fission track data from detrital samples belongs to Ballena-1X exploratory well from Salaverry Basin, Offshore Central Peru.

\begin{tabular}{|c|c|c|c|c|c|c|c|c|c|c|c|c|c|c|c|c|}
\hline Sample & $\begin{array}{c}\text { Depth } \\
(\mathrm{m})\end{array}$ & $N$ & $\begin{array}{c}\rho s \\
\left(\times 10^{5}\right)\end{array}$ & Ns & $\begin{array}{c}\text { pi } \\
\left(\times 10^{5}\right)\end{array}$ & $\mathbf{N i}$ & $\begin{array}{c}\rho d \\
\left(\times 10^{5}\right)\end{array}$ & Nd & $\begin{array}{c}\text { Central } \\
\text { Age (Ma) }\end{array}$ & $\pm 1 \sigma$ & $\begin{array}{c}X^{2} \\
(\%)\end{array}$ & $\begin{array}{c}\text { U Content } \\
\text { (ppm) }\end{array}$ & $n$ & $\begin{array}{c}\mathrm{Lm} \\
(\mu \mathrm{m})\end{array}$ & $\pm 1 \sigma$ & $\begin{array}{l}\text { Dpar } \\
(\mu \mathrm{m})\end{array}$ \\
\hline B1 & $207-225$ & 30 & 2.02 & 128 & 5.81 & 368 & 10.08 & 10075 & 53.0 & 5.4 & 64 & 7.3 & 15 & 10.72 & 1.47 & 3.4 \\
\hline B7 & $945-963$ & 14 & 11.1 & 277 & 22.3 & 557 & 10.08 & 10075 & 72.9 & 6.0 & 11 & 28.1 & 27 & 11.21 & 0.93 & 2.9 \\
\hline
\end{tabular}

$N$ : number of grains analysed to determine track densities; $\rho_{\mathrm{s}}$ : measured spontaneous track density; $N_{\mathrm{s}}$ number of spontaneous tracks counted; $\rho_{\mathrm{i}}$ measured induced track density;

$\mathrm{Ni}$ : number of induced tracks counted; $\rho \mathrm{d}$ : track density measured in glass dosimeter; $\mathrm{Nd}$ : number of tracks counted in determining $\rho \mathrm{d} ; \mathrm{x}^{2}$ : Chi-square probability;

$\mathrm{n}$ : number of confined tracks lengths measured; Lm: mean track length. Note: AFT ages were calculated by $\mathrm{C}$. Oliveira using $\zeta$-CN5 $=303.39$.

\section{Sample description}

B1: Conglomeratic sandstone of Pliocene-Pleistocene age

B7: Fine grain quartz sandstone of Upper Eocene age 


\subsection{DISCUSSION}

In the Cenozoic succession of the Salaverry Basin, seismic data allows the recognition of twelve seismic sequence boundaries defining eleven seismic sequences. The geometry of the seismic sequences, thickness variations and seismic facies are integrated with well and core data allowing an improved interpretation of the tectonosedimentary evolution and gross depositional environments in each sequence. This geological framework and the available dataset better defines the implications for hydrocarbon exploration in the study area, which are described below.

\subsubsection{TECTONO-SEDIMENTARY EVOLUTION}

Based on the location of depocentres, seismic facies distribution, reflection terminations and the knowledge of the Andean Orogenic cycle, the S1-S11 seismic sequences are interpreted to reflect major changes in the basin evolution. During S1 time (41.2 - 33.9 Ma) occurs the initial stage of basin formation controlled by northwest and northeast trending high angle normal faults that define the SWD (Figs. 3, 5C, 6D, and 8). This depocentre was filled out by deltaic/shelf deposits that progradated to the west having as source area the Precambrian metamorphic rocks belongs to the Paracas Arch (Figs. 10, 17 and 19A). These deposits conform the S1 sequence (Fig. 17). After the deposition of lower Oligocene rocks, the Incaic III tectonic phase added to the strong sea-level fall took place and triggering the erosion of the entire Oligocene sedimentary record and the uppermost section of the upper Eocene rocks (Fig. 17). Thus, this tectonic event is well identified through the AFT analysis and is related to plates reorganization after break-up of the Farallon plate (Ramos, 2009) (Fig. 16).

During S2 time (23.1 - 15.9 Ma) the basin subsided continuously while the sealevel rose (Fig. 4), thereby developing two main depocentres: the SWD extending to the east and another located to the north (Fig. 8). The deposition of S2 sequence was dominated by deltaic/shoreface deposits (Fig. 4 and 19B). Moreover, the S2 reflection packages onlaps and thins towards the flanks of a structural high located in the south, which is likely active during S2 time (Fig. 8). Likewise, during this time Quechua I tectonic phase was active controlling a prominent intrabasinal high and uplift of the eastern margin (Fig. 8). In addition, mass transport deposits associated to channel/canyon fill and gravity flows are recognized westward (Fig.10). 
During S3 time $(15.9$ - $14 \mathrm{Ma})$ continues the sea-level rise and the deposits retrograde landward (Fig.17). Likewise, the deposition is controlled by the inherit paleotopography and thereby don't show marked thickness trends along the basin (Fig. 8). In this way, the S3 sequence would consist of shoreface deposits at the base overlain by pelagic/hemipelagic deposits to the top (Figs. 4 and 17). Tectonism could be controlling the structural highs where the $\mathrm{S} 3$ sequence is absent (Fig. 8).

During S4 time (14 - 11.6 Ma) the basin subsided product of the Andean Orogeny while the sea-level fell (Figs. 4 and 8). The S4 sequence onlaps on the eastern margin and subtle downlaps westward showing a progradational pattern to the west during sealevel fall (Figs. 5B, 7 and 13). S4 consist of deltaic/shoreface deposits eastward, turbidites related to pelagic/hemipelagic deposits at basin foredeep (Fig. 20A and B) and punctuated mass transport deposits westward on slope environment (Fig. 10). On the other hand, consistently subtle truncation of reflections along the basin foredeep could be related to bottom currents erosion whereas angular truncation over the western margin is product of Paracas Arch uplift (Fig. 7). In addition during this time begin the initial stage of normal faults inversion, as formation of intrabasinal highs (Fig. 12C-F), this compression event would be related to the Nazca ridge subduction (Fig. 17).

During S5 time (11.6 - $7 \mathrm{Ma})$ the subsidence in the basin is controlled by the Andean uplift, and hence the basin foredeep is very well defined (Figs. 5A, 5B, and 8). On the SWD margin an inherit paleohigh is likely active limiting the deposition in this area (Fig. 8). The S5 onlaps on the eastern margin over the PCB and consistently donwlaps westward, similarly to S4 sequence it reflect a progradational pattern to the west during sea-level rise (Figs. 5B, 7 and 13). S5 sequence consist of deltaic/shoreface deposits eastward, turbidites related to pelagic/hemipelagic deposits at basin foredeep (Fig. 20C and D) and mass transport deposits westward on slope environment (Fig. 17). Tilting and strong erosion over the Paracas arc suggest syn-depositional uplift (Figs. 5B and 7), and on the other hand the formation of intrabasinal highs continues product of normal fault inversion (Fig. 12C-F). Both events are correlated with Quechua II tectonic phase (Fig. 17). 


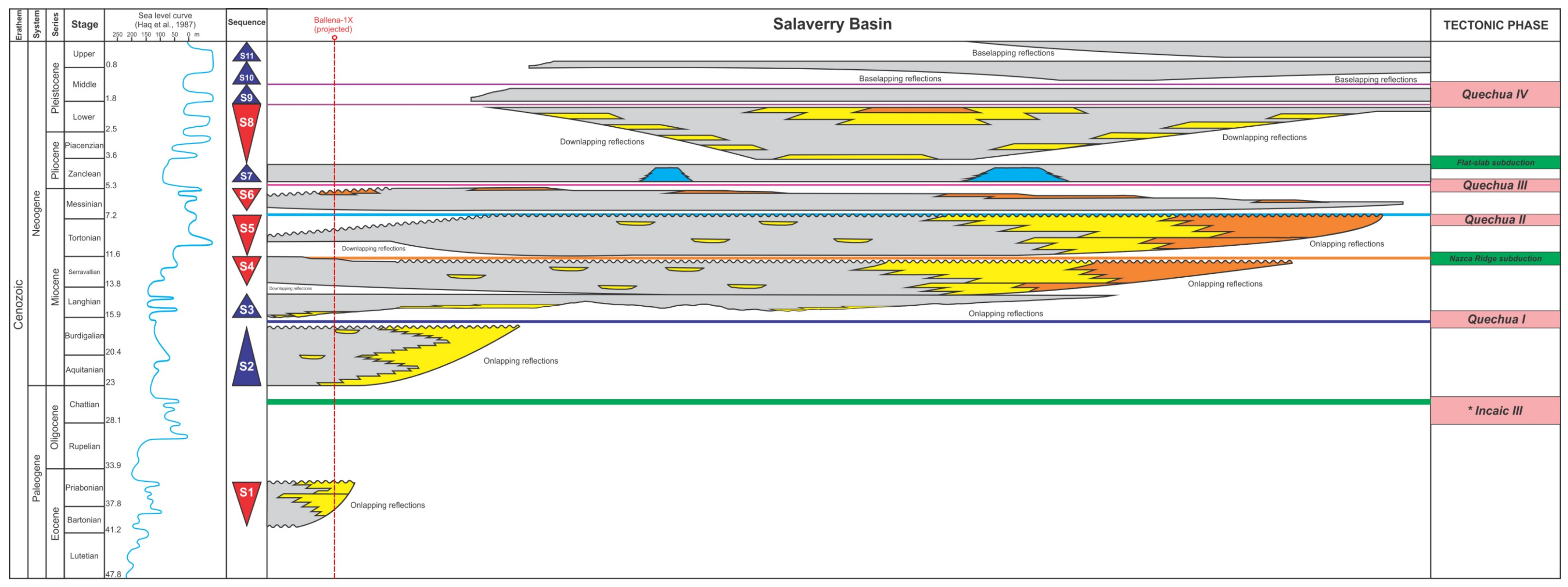

$\bigvee_{\text {Regradational }}^{\text {Stacking pattern }}$

$\square$ Delta plain / fluvial deposits

$\square$ Shoreface / Delta Front deposits

$\square$ Pro-delta / offshore deposits

$\smile$ Turbidite deposits

$\square$ Carbonate patch reef

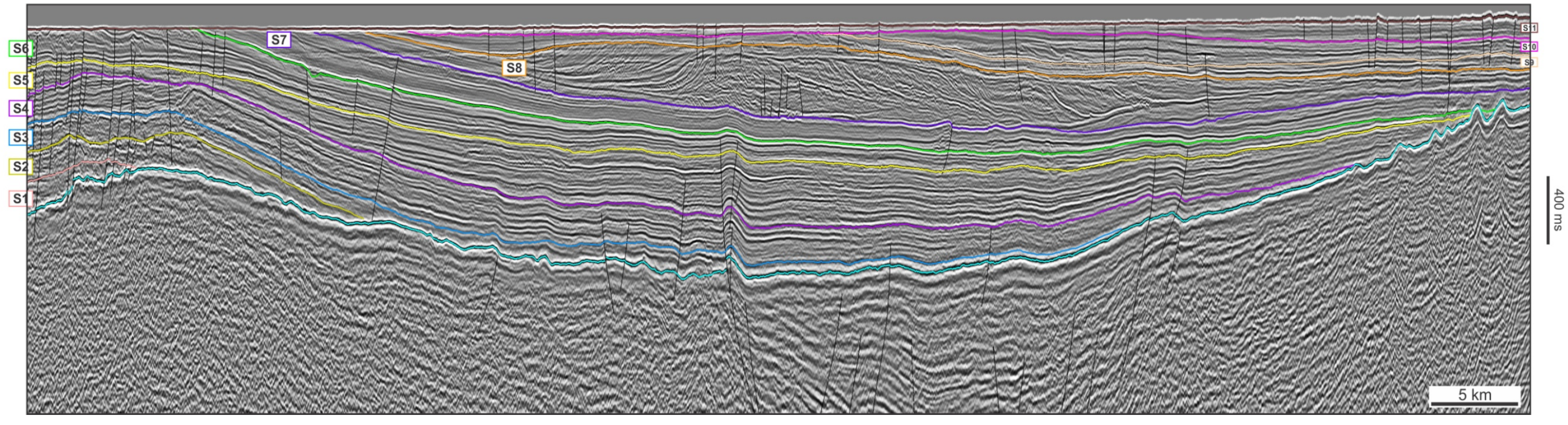

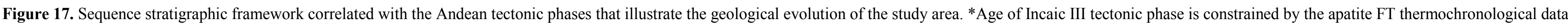


During S6 time (7 - $5 \mathrm{Ma}$ ), the tectonic activity stops and hence the Paracas Arch uplift, this fact also generates a slow subsidence in the basin (Fig. 9). The sedimentation was predominantly controlled by the paleotopography and the sea-level rise (Haq et al., 1987). In addition, subtle progradational pattern to the east and west is recognized by toplap terminations against SB7 during sea-level rise. S6 sequence consists of shelf deposits overlain by nearshore deposits (Figs. 4 and 17).

The transition to S7 time in the basin is marked by change in local tectonics (Quechua III tectonic phase) and rise of sea-level (Fig. 17), factors which together generate favorable conditions for the formation of patch reefs in the basin (James and Wood, 2010) (Figs. 11, 20E and 20F; Table 1, SF11). The initiation of reef growth occurs during a rise of sea-level after change in local tectonics, in isolated areas in the basin (Fig.11), where minimal siliciclastic input favors the development of their basic constructional elements: microbes, calcareous algae, metazoans, and sin-sedimentary carbonate cements (James and Wood, 2010). Later the shallow marine settings change to basin deepening, developing pelagic/hemipelagic fine-grained deposits related to a TST (Fig. 17)

During S8 time (4 - $2 \mathrm{Ma})$ the basin suffered great subsidence product of the Andean Orogeny related to flat-slab subduction stage (Figs. 5A, 5B 9 and 17). This generated accommodation space was filled out by at least 3 progradational events from SE to NW. Only one of them has northeast trending, which suggest that some centralsouth portion of the Paracas Arch would act as source area (Figs. 9, 11 and 15). The S8 consist of shelfal progradation deposits developed during sea-level rise decreasing along the basin foredeep (Figs. 9 and 15). These deposits are characterized by aggradationprogradation-degradation stratal stacking patterns (Fig. 15C-D) and not well defined: forced regression (offlap breaks that step down without topsets onlapping the inherited depositional profile) and basinward shift of coastal onlap (topsets onlapping the inherited depositional profile) (Neal and Abreu, 2009). Likewise westward, the submerged Paracas Arch uplift controlled the deposition and constrained the S8 sequence to Salaverry Basin and SWD (Figs. 5 and 9).

After the S8 time a period of non-deposition and subtle erosion is marked by baselap above the SB9 (Fig. 15). From this time and during the last $2 \mathrm{Ma}$, the Isochron maps show the continuous uplift of the submerged Paracas Arch until the S11 time (Fig. 
9), which constrain the geographic extent of the last three sequences (S9 - S11) increasingly eastward. At the same time, the main depocentre migrates from the central sector to the south of the study area (Fig. 9). These basin settings would be related to the Quechua IV tectonic phase (Fig. 17). In addition, between S9, S10 and S11 sequences, periods of non-deposition and erosion are evidenced by regional baselap (Fig. 13). Each of these sequences consists predominantly of pelagic/hemipelagic deposits (Fig. 17).

\subsubsection{NEW PETROLEUM SYSTEM INSIGHTS}

Previous exploratory works define two petroleum systems in the Salaverry Basin: Upper Jurassic-Cenozoic (.) and Albian-Cenozoic (.) (see Valencia et al., 2011 for detailed explanation), but don't include a detailed seismic stratigraphic assessment. Indeed, the present work is focused in the exploration of new petroleum system elements within the entire Cenozoic succession. In this way, the following section describes the results and the contribution for hydrocarbon exploration in the study area.

\subsubsection{HYDROCARBON EVIDENCES}

Since 1995 to 2000, the identification of oil shows in Morsa-1X and Lobos-1X exploratory wells drilled in Trujillo Basin added to 6 oil seeps recognized, sampled and analyzed in the same basin, evidence at least one active petroleum system around the Salaverry Basin (Minguito et al., 1997; Quesada et al., 2000). In the same way, the reconnaissance of oil slick derived from SAR data mapping records 3 oil seeps inside Salaverry Basin, which were sampled and analyzed. These oil seeps become a trustful proof of the generated hydrocarbon in the basin. In addition, they are spaced along the basin and related to potential kitchens recognized by seismic interpretation (Valencia et al., 2013; Timoteo et al., 2014). Although none of these oil seeps is located in the study area, two potential kitchens and several DHIs (Direct Hydrocarbon Indicators) have been recognized across the $2 \mathrm{D}$ seismic surveys (Figs. 18 and 22B).

In the present work, analysis of 2D seismic reflection data reveals shallow and deep gas indicators including gas chimneys, enhanced reflections and bright spots. Even though it was not an objective for our work, during the seismic stratigraphic interpretation it was possible recognize at least 50 locations with this kind of anomalies: 

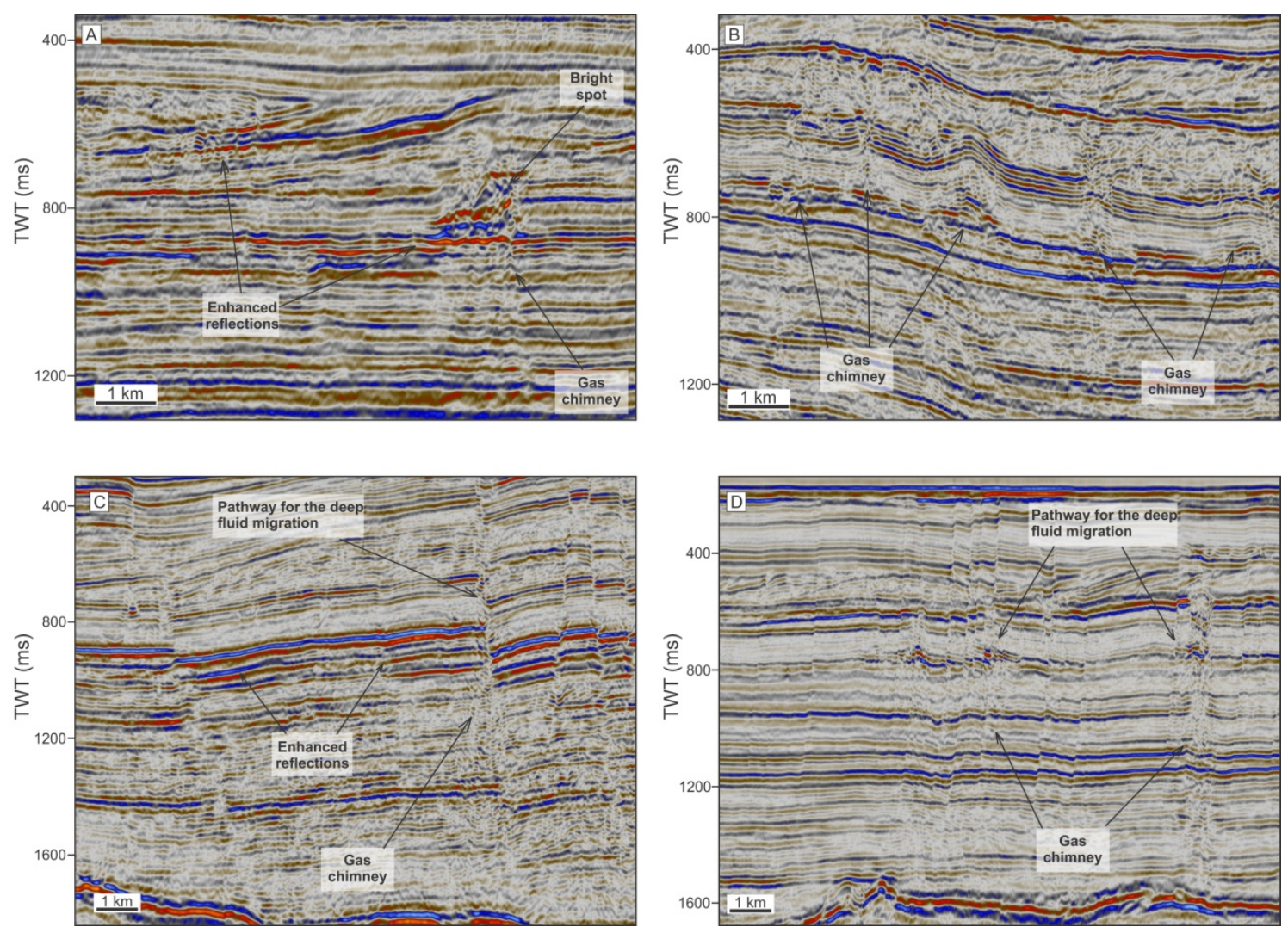

Figure 18. Seismic profiles showing different type of DHIs recognized in the study area. A) Potential shallow gas accumulations marked by bright spots and enhanced reflections. B) Fluid flow systems recognized as gas chimneys. C) and D) Leakage along faults, interpreted as pathways for deep fluid migration in the basin.

- Enhanced reflections: suggest the presence of free gas and they are characterized by very high amplitude reflections (Fig. 18A and C).

- Gas chimneys: these DHIs are identified as vertical or columnar zones of amplitude blanking related to fluid leakage within the subsurface. They have been observed in the study area (Fig. 18) and some of them could be associated with faults that would act as migration pathways for deep fluids (Fig. 18C and D).

- Bright spots: or very strong amplitude anomalies with negative polarity are also recognized and indicate the presence of gas (Fig. 18A).

- Acoustic masking: characterized by faint or absence of reflections has been recognized over structural highs and deeper zones in the basin. This conspicuous blanking suggests the absorption of acoustic energy in the overlaying gas-charged sediments. 
Origin of these shallow and deep gas could be by biogenic or thermogenic processes. Thermogenic gas requires vertical migration pathways as the faults recognized in Fig.18C and D.

\subsubsection{POTENTIAL RESERVOIR AND SEAL ROCKS}

Based on integrated seismic sequence analysis we interpret depositional systems and associated potential reservoir and seal rocks. In this way, the following section describes the key petroleum system elements recognized within the Cenozoic succession of Salaverry Basin.

\section{A) S1 Reservoir facies (Upper Eocene)}

The overall wedge geometry together with the consistent onlap on the Paracas Arch of the hummocky seismic facies (SF10, Fig. 8) are interpreted as shallow marine sedimentation related to deltaic/shelf depositional system. Analogous interpretation can be derived from core lithofacies of Ballena-1X, along the hummocky seismic facies interval (SF10), which depict a deltaic/shelf depositional system comprised by different sandstones facies that show favorable petrographic characteristics and good petrophysical properties as reservoir rocks (Fig. 19A) (see Minguito et al., 1997 for an overview of petrophysical evaluation).

\section{B) S2 Reservoir facies (Lower Miocene)}

The hummocky seismic facies (SF10), together with wedge and sheet draping geometry, and onlap on the Paracas Arch and on the PCB, dominate the sedimentary infill of depocentres during S2 time (Figs. 8 and 10) and thus suggest deltaic/shelf depositional system likely associated with flysch-like delta front deposits basinward. In the same way, sedimentary facies described in cores from Ballena-1X, along the S2 sequence interval, correspond to marine claystones and shales interbedded with deltaic/shelf cross-bedded sandstones and flysch-like massive sandstones, which suggest good characteristics as reservoirs rocks (Fig. 19B). 


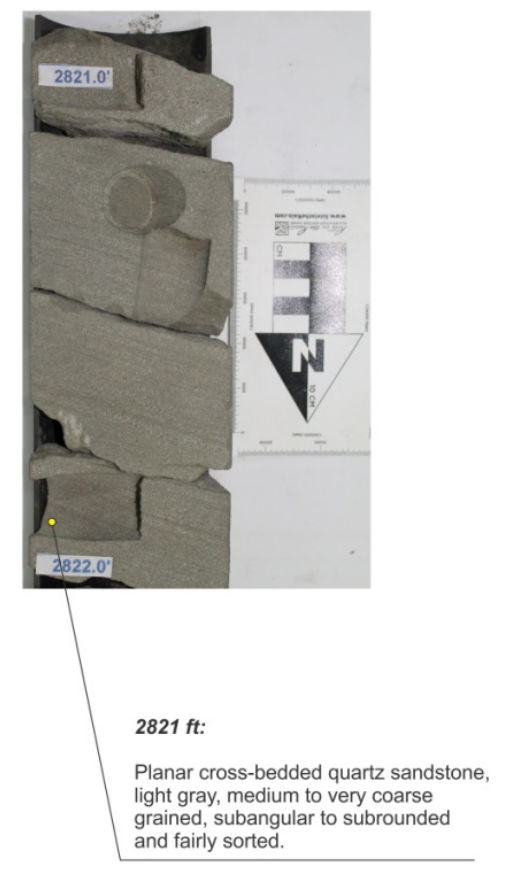

A

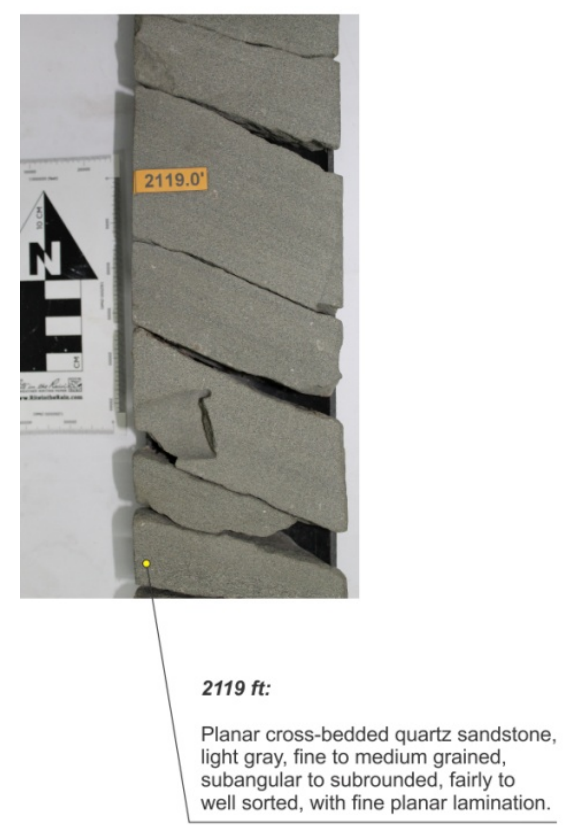

B
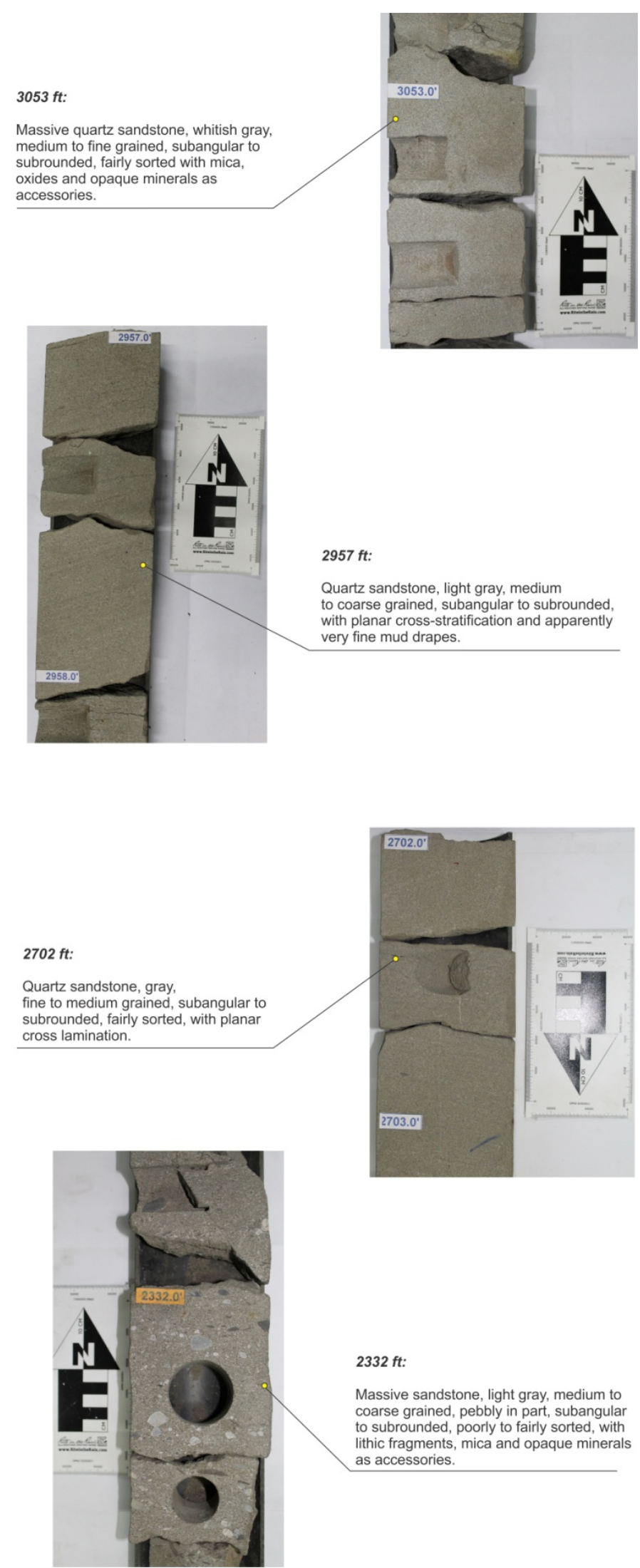

Figure 19. Core lithofacies from Ballena-1X exploratory well. A) Favorable reservoir facies within the Upper Eocene Verdun formation (S1 sequence) are described. B) Interbedded sandstones showing better conditions to potential reservoir rocks within the Lower Miocene Heath Formation ( $\mathrm{S} 2$ sequence) are described. 


\section{C) S4 Reservoir facies (Middle Miocene)}

In the S4 time depocentre, the sedimentary succession is dominated by SF4 seismic facies, with sheet drape external geometry and concordant boundaries. They are interpreted as pelagic/hemipelagic shales and claystones interbedded with turbiditic sandstones (Figs. 8 and 10). These turbidite sandstone deposits have been recognized in the $3 \mathrm{D}$ seismic cube, where amplitude and variance time slices better define the submarine channel system that could host potential reservoir rocks (Fig. 20A and B). On the other hand, towards the east, wedge-like continuous narrow fringe of SF3 and SF6 reflections that consistently onlap on the PCB are interpreted as shoreface/deltaic deposits with favorable conditions to potential reservoir rocks (Figs. 10 and 22A).

\section{D) S5 Reservoir facies (Upper Miocene)}

The internal configuration and external geometry of S5 seismic facies are most similar to those recognized in S4 seismic sequence (Fig. 10), which suggests pelagic/hemipelagic sedimentation associated with low density turbidity currents during S5 time. This gross depositional system interpretation has been corroborated in time slices from 3D seismic cube, where submarine channel system is subtle defined in the variance map (Fig. 20C) but is better defined in RMS amplitude map (Fig. 20D). Both seismic attribute maps have strong correlation and suggest that this channel system could host turbidite sandstones as potential reservoir rocks.

\section{E) S7 Reservoir facies (Pliocene)}

Mounded seismic facies bodies (SF12) have been recognized in different parts of S7 time depocentre (Fig. 11) and are interpreted as carbonate buildups likely pinnacle reefs due to its internal reflections are subparallel differing from cone-in-cone architecture reflections conspicuous from volcanic buildups. These patch reefs are better defined in the amplitude and variance time slices (Fig. 20E, F), where carbonate factory is represented by several semi-circular bodies with low to high amplitudes. This kind of organic rocks may represent important reservoirs in the basin. 


\section{F) S8 Reservoir facies (Pliocene - lowermost Pleistocene)}

The S8 sequence is marked by at least 3 progradational events that as whole compose a major delta that progradates from SE to NW. The south-central sector of S8 is characterized by sigmoid/oblique seismic facies (SF8), which suggest sedimentation controlled by suspension and therefore predominance of very fine-grained facies in the clinoforms (Fig. 11). On the other hand, the central-north sector of S8 is dominated by concave upward seismic facies (SF9), which suggest sedimentation controlled by traction related to a shelfal progradation (also known as deltaic progradation) (Figs. 11 and 15). According with this, the clinoform topsets would contain a combination of delta plain and fluvial deposits whereas the clinoform tops (offlap break) are interpreted as combination of distributary mouth bar and shoreface deposits, which have been recognized in amplitude and variance time slices (Fig. 20I, J). In addition, the clinoform foresets are interpreted as pro-delta and offshore marine deposits likely composed by shales, mudstones and siltstones. Finally, the clinoform bottomsets show low amplitude, baselap and lens-like external form (Fig. 15), and are interpreted as turbidite lobes. Each of these turbidite lobes is better defined in amplitude and RMS amplitude time slices, and they could host potential reservoir rocks (Fig. 20G, H).

\section{G) Favorable Seal facies}

The integrated seismic sequence analysis performed in the study area allows interpret pro-delta/shelf deposits within S1, S2 and S8 seismic sequence, and pelagic/hemipelagic deposits within S4 and S5 seismic sequence. In this way, pro-delta/shelf shales and mudstones together with pelagic/hemipelagic shales, claystones and siltstones could act as internal seal rocks for potential reservoir rocks identified within each seismic sequence above mentioned.

In addition, seismic facies distribution from S3, S6, S7, S9, S10 and S11 sequences are mostly characterized by parallel, continuous, variable amplitude reflections, suggesting dominance of pelagic/hemipelagic sedimentation during each sequence time (Fig. 11). In this way, interpreted pelagic/hemipelagic deposits are the best place to look for regional seal rocks within S3, S6, S7, S9, S10 and S11 sequences; due to they would be composed predominantly by shales, claystones, mudstones and siltstones. 

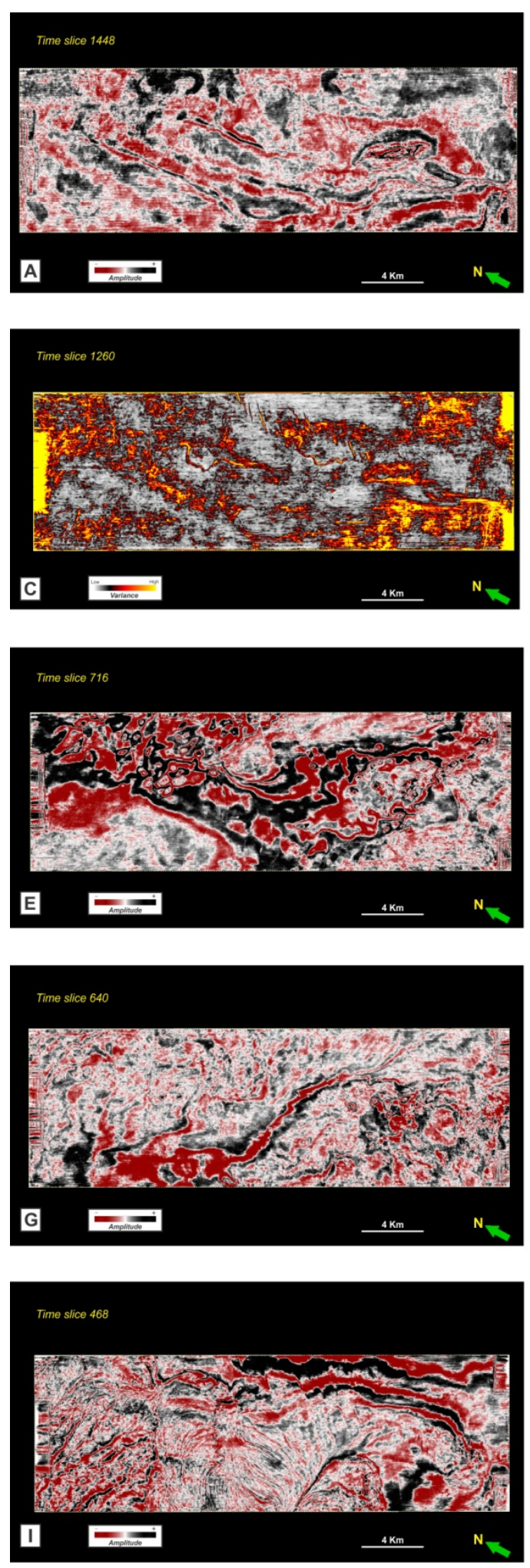
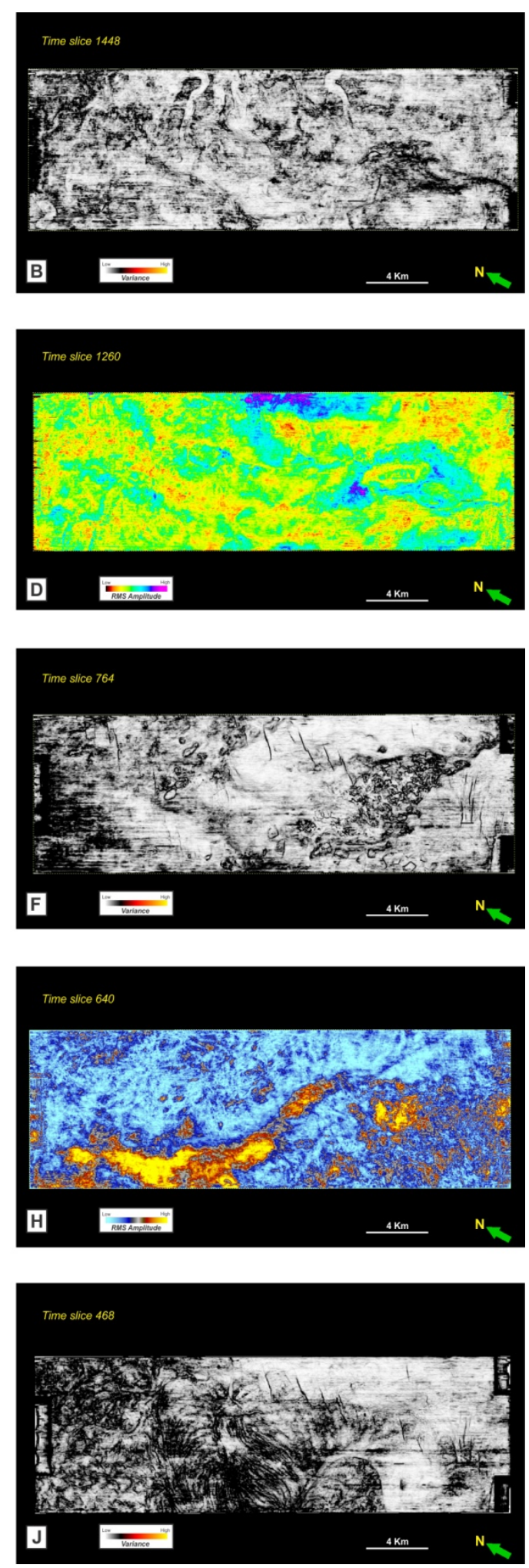

Figure 20. Seismic attribute maps from 3D seismic time slices in the study area: A) Amplitude and B) variance slices showing submarine channel system within S4. C) Variance and D) RMS amplitude slices, which better define another submarine channel system within S5. E) Amplitude and F) variance slices showing carbonate reefs within S7. G) Amplitude and H) RMS amplitude slices showing turbidite lobe at clinoform bottomsets from S8. I) Amplitude and $\mathrm{J}$ ) variance slices showing deltaic/shoreface deposits at clinoform tops (offlap break) from S8. Refer to Figure 2 for 3D seismic cube location. 


\subsubsection{POTENTIAL PLAYS}

Nine potential plays (a series of traps) have been interpreted to provide a range of unproved play types that may exist in central portion of Salaverry Basin, Offshore Peru (Fig. 21). They will be described from east to west. Many of the most relevant trap styles are shown on seismic profiles (Fig. 22).

\section{A) Fractured volcanic-igneous Play (Figs. 14 and 21)}

This play is constrained to the Volcanic arc and Coastal Batolith domain (Fig. 21), and is conformed by shallow structural highs. Each of these highs is controlled by the morphostructure and/or normal faults on its flanks, and they are overlaying by PlioPleistocene shales (Fig. 14).

\begin{tabular}{ll}
\hline Area & $160-300 \mathrm{~km}^{2}$ \\
\hline Type of traps & $\begin{array}{l}\text { Geomorphic and piercement related to volcanic and intrusive bodies, } \\
\text { respectively }\end{array}$ \\
\hline Main reservoirs & Cretaceous to Paleogene fractured volcanic and igneous rocks (PCB) \\
\hline Source rocks & Upper Jurassic marine shales, Albian marine shales \\
\hline Critical aspects & Fracture porosity and permeability, migration-preservation \\
\hline
\end{tabular}

\section{B) Stratigraphic Play (Figs. 21 and 22A)}

This play includes a variety of stratigraphic traps: onlap of Middle (S4) and Upper Miocene (S5) sandstones on Cretaceous volcanic rocks and shales, and Middle Miocene sandstones (S4) subcropping below Upper Miocene shales (Fig. 22A). Exploratory wells proposed to test structural traps would test some of these concepts.

\begin{tabular}{ll}
\hline Area & $660-800 \mathrm{~km}^{2}$ \\
\hline Type of traps & Onlaps and subunconformity truncations \\
\hline Main reservoirs & $\begin{array}{l}\text { Middle and Upper Miocene sandstones (belongs to S4 and S5 } \\
\text { sequences, respectively) }\end{array}$ \\
\hline Source rocks & Upper Jurassic marine shales, Albian marine shales \\
\hline Critical aspects & Mapping of trap and lateral seal \\
\hline
\end{tabular}




\section{C) Mesozoic Graben Play (Figs. 21 and 22B)}

This play is a major Mesozoic graben system developed along the basin axis. This system ranges from 20 to $30 \mathrm{~km}$ wide and extends more than $100 \mathrm{~km}$ in the study area (Fig. 21). The level of inversion is relatively low, but mainly has influence in the master faults over the graben margins; and is associated with tilted blocks rotated during the extensional phase. Onlaps against Precambrian and Paleozoic basement and subunconformity toplaps constitute the main traps in this play (Fig. 22B).

\begin{tabular}{ll}
\hline Area & $700-1400 \mathrm{~km}^{2}$ \\
\hline Type of traps & Partially inverted normal fault, onlaps and toplaps \\
\hline Main reservoirs & $\begin{array}{l}\text { Lower Cretaceous fractured sandstones, Upper Cretaceous fractured } \\
\text { limestones and volcanic rocks (PCB) }\end{array}$ \\
\hline Source rocks & Upper Jurassic marine shales, Albian marine shales \\
\hline Critical aspects & $\begin{array}{l}\text { Fracture porosity and permeability for Lower and Upper Cretaceous } \\
\text { reservoirs }\end{array}$ \\
\hline
\end{tabular}

\section{D) Embedded Turbidite Lobe Play (Figs. 21 and 22C)}

The Embedded Turbidite play corresponds to the basal section of the Plio-Pleistocene major delta extending along the basin foredeep in the study area (Fig. 15). This stratigraphic play ranges from 10 to $30 \mathrm{~km}$ wide and extends more than $70 \mathrm{~km}$ in the study area (Fig. 21). In addition, it has minor faulting and is located close to the onshore Santa and Casma fluvial systems. Shelfal progradation during Upper Pliocene to Lower Pleistocene sea-level rise decreasing generated shoreface and distributary mouth bars facies at shelf follow to pro-delta/offshore shales on the slope and finally distal turbidite lobes basinward. Each of these turbidite lobes is a potential trap due to they are encased by shales derived from previous and subsequent progradational cycles (Figs. 20G, 20H and 22C). Moreover, associated shallow marine sandstones facies could be represent an additional economic target (Figs. 20I, 20J and 22C). Exploratory wells proposed to test structural highs would test these concepts. 


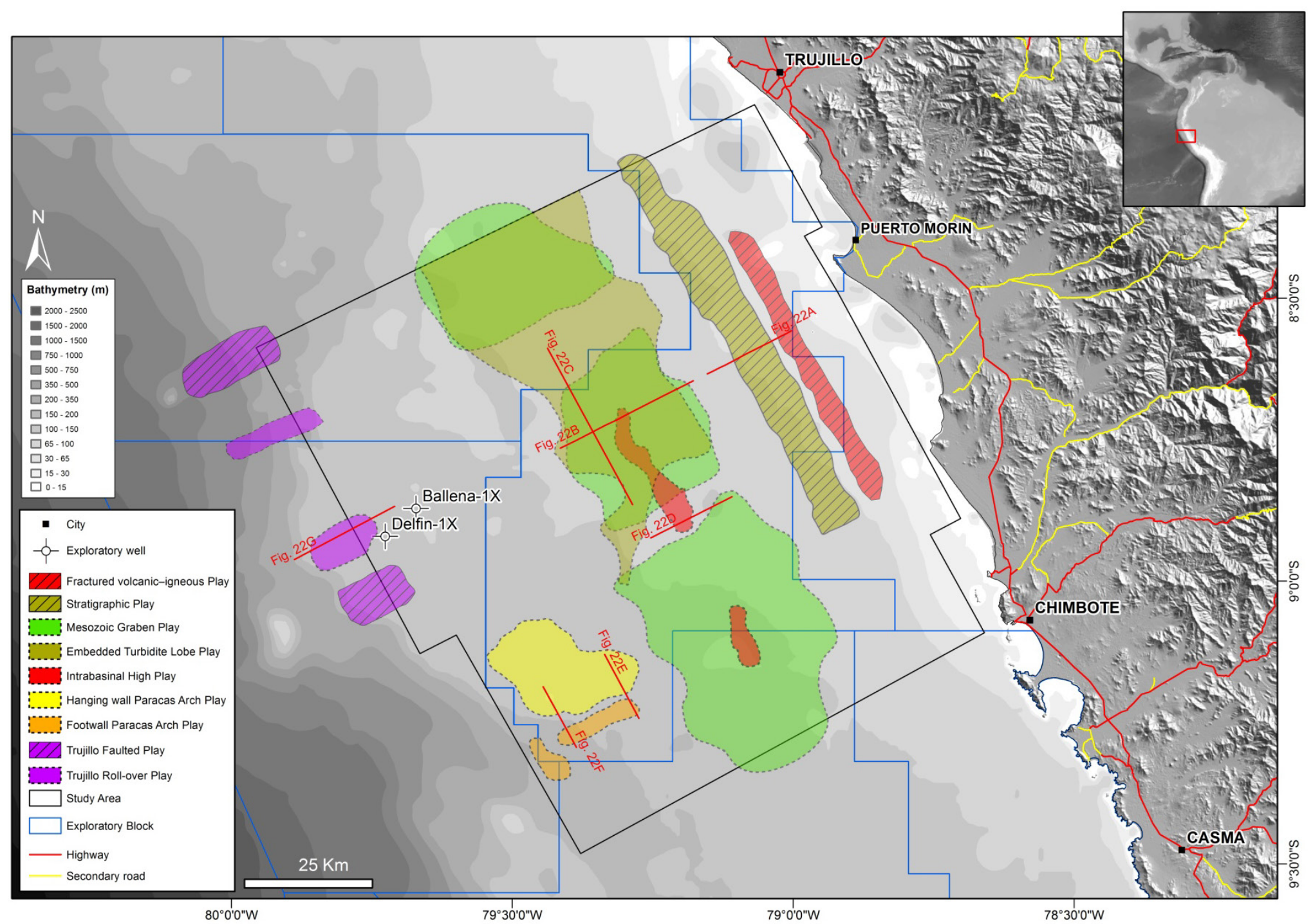

Figure 21. The colored polygons shown offshore represent the mean areal extent of each potential play identified in the central portion of Salaverry Basin within the study area. The bathymetry related to each potential play is shown in grayscale. 


\begin{tabular}{ll}
\hline Area & $1200-1700 \mathrm{~km}^{2}$ \\
\hline Type of traps & Embedded turbidite lobes encased in shales \\
\hline Main reservoirs & Pliocene to lowermost Pleistocene turbiditic sandstones (S8) \\
\hline Source rocks & Upper Jurassic marine shales, Albian marine shales \\
\hline Critical aspects & Trap-charge timing, target depths \\
\hline
\end{tabular}

\section{E) Intrabasinal High Play (Figs. 21 and 22D)}

The Intrabasinal High play includes different northwest-trending structural highs extending in the basin foredeep. The trap style corresponds to anticline fold, product of fault propagation of Miocene inverted normal faults, ranges from 12 to $26 \mathrm{~km}$ extension (Figs. 21 and 22D). A Pliocene to Holocene phase of hydrocarbon generation and expulsion favors the presence of hydrocarbons in these traps. Exploratory wells proposed to test these structural traps would drill five potential reservoir rocks becoming this play in a very attractive exploratory target (Fig. 22D).

\begin{tabular}{ll}
\hline Area & $50-100 \mathrm{~km}^{2}$ \\
\hline Type of traps & Fault propagation folds \\
\hline Main reservoirs & $\begin{array}{l}\text { Middle (S4) and Upper Miocene (S5) sandstones, Pliocene } \\
\text { carbonates (S7) and sandstones (S8), Upper Cretaceous fractured } \\
\text { limestones and volcanic rocks (PCB) }\end{array}$ \\
\hline Source rocks & Upper Jurassic marine shales, Albian marine shales \\
\hline Critical aspects & Reservoir quality, vertical closure \\
\hline
\end{tabular}

\section{F) Hanging wall Paracas Arch Play (Figs. 21 and 22E)}

The Hanging wall Paracas Arch play represents the upthrown block related to a northeast-trending major normal fault belongs to Trujillo Fault System (Figs. 3, 21 and 22E). Likewise, this prominent structural high is strongly faulted generating minor blocks. Each of the upthrown sides of these normal faulted blocks is a potential trap associated to at least three potential reservoirs overlain by 200 to $300 \mathrm{~m}$ thickness of seal rocks (Fig. 22E). Present-day oil and gas generation and expulsion favor this area, where several DHIs have already been recognized in the seismic data.

\begin{tabular}{ll}
\hline Area & $350 \mathrm{~km}^{2}$ \\
\hline Type of traps & Normal faulted blocks \\
\cline { 1 - 2 }
\end{tabular}




\begin{tabular}{ll}
\cline { 2 - 2 } Main reservoirs & $\begin{array}{l}\text { Middle (S4) and Upper Miocene (S5) sandstones, and Precambrian } \\
\text { fractured metamorphic rocks (PCB) }\end{array}$ \\
\hline Source rocks & Upper Jurassic marine shales, Albian marine shales \\
\hline Critical aspects & Migration pathways, reservoir integrity \\
\hline
\end{tabular}

\section{G) Footwall Paracas Arch Play (Figs. 21 and 22F)}

The Footwall Paracas Arch play is constrained to the north margin of southwestern depocentre (SWD) and represents the downthrown block related to the same major normal fault before described in the Hanging wall play (Figs. 3, 21 and 22F). The listric behavior of this major normal fault generated a significant roll-over anticline, which in turn is faulted. High-angle normal faults compartmentalize the potential reservoirs in minor faulted blocks and likewise generate migration pathways for hydrocarbon generated in the SWD (Fig. 3, 21 and 22F). Each of these faulted anticline blocks is a potential trap. Exploratory wells proposed to test the Hanging wall play (Fig. 22E) would test these concepts (Fig. 22F).

\begin{tabular}{ll}
\hline Area & $40-100 \mathrm{~km}^{2}$ \\
\hline Type of traps & Faulted Roll-over anticline \\
\hline Main reservoirs & $\begin{array}{l}\text { Middle (S4) and Upper Miocene (S5) sandstones, Pliocene } \\
\text { sandstones (S8), and Precambrian fractured metamorphic rocks } \\
\text { (PCB) }\end{array}$ \\
\hline Source rocks & Upper Jurassic marine shales, Albian marine shales \\
\hline Critical aspects & Trap-charge timing, reservoir presence and quality \\
\hline
\end{tabular}

\section{H) Trujillo Faulted Play (Fig. 21)}

The Trujillo faulted play is mainly constrained to the Trujillo Basins but its eastern flank is located on the western margin of Salaverry Basin inside the study area (Fig. 21). High-angle northwest-trending normal faults generated an array of en echelon blocks with subtle tilting in part, which are potential traps. The sizes of untested structures in this play are such that they could hold a hydrocarbon accumulation. However, the quality of unproven potential reservoirs is poorly known. 

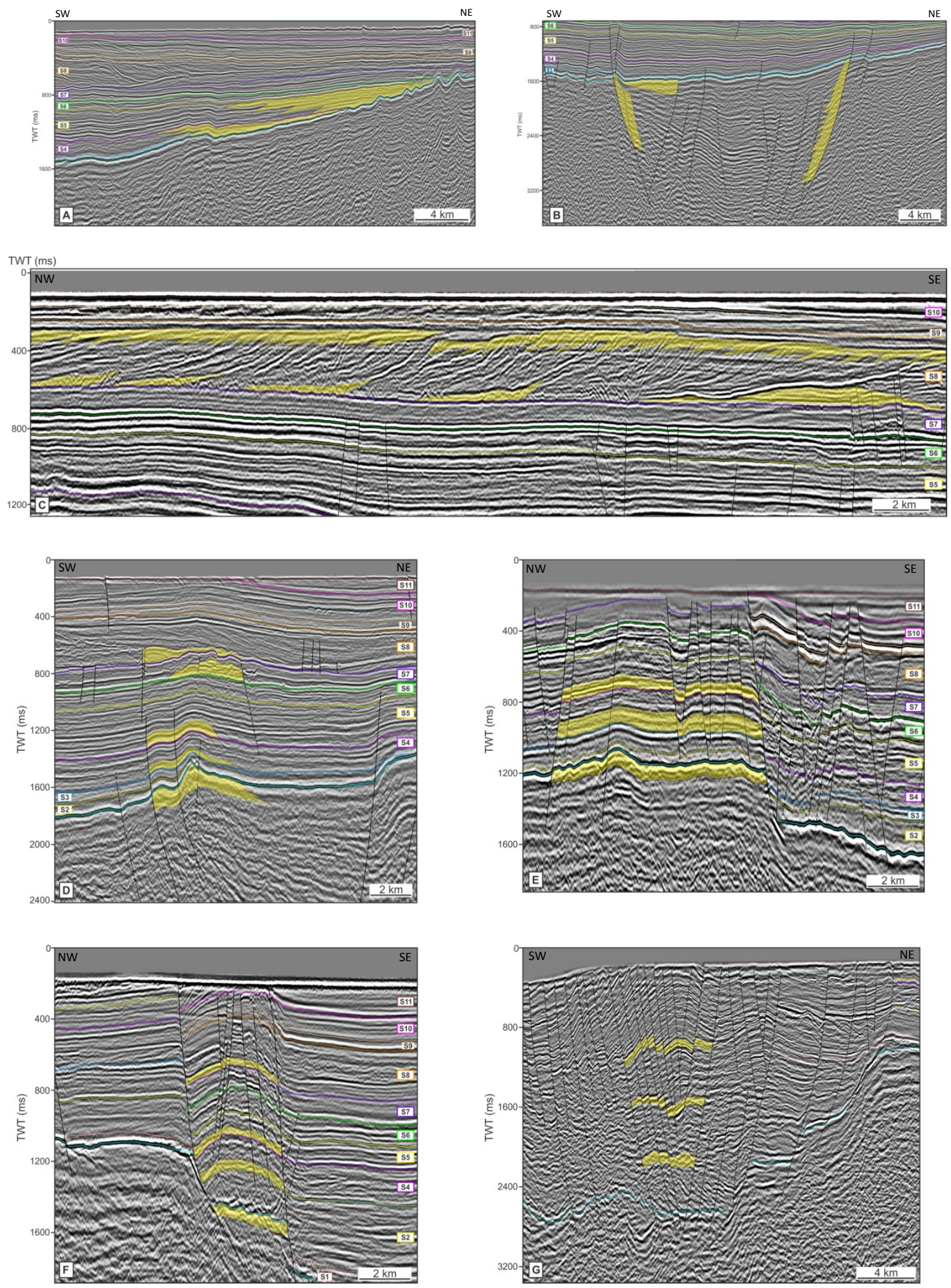

Figure. 22. Seismic profiles showing many of the most relevant trap types recognized in the study area. A) onlaps and subunconformity truncations. B) Partially inverted normal fault, onlaps and toplaps. C) Embedded turbidite lobes encased in shales. D) Fault propagation folds. E) Normal faulted blocks. F) Faulted Roll-over anticline. G) Faulted Roll-over anticline related to major listric faults. Refer to Figure 21 for seismic profile locations. 


\begin{tabular}{ll}
\hline Area & $100-250 \mathrm{~km}^{2}$ \\
\hline Type of traps & Normal faulted blocks and tilted blocks \\
\hline Main reservoirs & Eocene and Miocene turbiditic sandstones \\
\hline Source rocks & Albian marine shales \\
\hline Critical aspects & Reservoir presence and quality \\
\hline
\end{tabular}

\section{I) Trujillo Roll-over Play (Figs. 21 and 22G)}

The style, size, and distribution of the anticlinal structures conform faulted roll-over anticlines as a structural play. The folded structures range from 12 to $18 \mathrm{~km}$ wide and likely extend $30 \mathrm{~km}$ (Fig. 21). At least two structural traps, containing three potential reservoir rocks each one, have been recognized around the study area in the Trujillo basin (Figs. 21 and 22G). These traps comprise folded Eocene to lower Miocene turbiditic sandstones interbedded with shales that form internal seals. In this type of play, fracture zones and fault systems associated with the Neogene extension could provide the main migration pathways for hydrocarbons sourced from Cretaceous marine shales (Fig. 22G). All of these structures could represent significant to major hydrocarbon fields (Chierici, 1995).

\begin{tabular}{ll}
\hline Area & $70-160 \mathrm{~km}^{2}$ \\
\hline Type of traps & Faulted Roll-over anticline related to major listric faults \\
\hline Main reservoirs & Eocene and Miocene turbiditic sandstones \\
\hline Source rocks & Albian marine shales \\
\hline Critical aspects & Reservoir presence and quality \\
\hline
\end{tabular}




\subsection{CONCLUSIONS}

- The Cenozoic succession in the central portion of Salaverry Basin is best subdivided into eleven seismic sequences: 3 second-order sequences (S1, S2 and S5) and 8 third-order sequences (S3, S4, S6-S11). All of them are bounded by twelve seismic sequence boundaries.

- AFT dating and modeling, from detrital borehole samples (B1 and B7), evidence an uplift event in the basin at ca. $25 \mathrm{Ma}$ that correlates with the strong Oligocene sealevel fall, which triggered the erosion of Oligocene rocks and the uppermost section of Upper Eocene deposits. These uplift event is well constrained in the offshore section and correlated with Incaic III tectonic phase, defined in the Andean mountains.

- Within the seismic sequences S1, S2, S4, S5, S7 and S8 have been interpreted key depositional systems that could host potential reservoir rocks:

S1: Deltaic/shelf sandstones (Upper Eocene).

S2: Deltaic and flysch-like sandstones (Lower Miocene).

S4: Turbidite sandstones related to pelagic/hemipelagic deposits (Middle Miocene).

S5: Turbidite sandstones related to pelagic/hemipelagic deposits (Upper Miocene).

S7: Carbonate patch reefs (Pliocene).

S8: Turbidite lobe sandstones and deltaic/shoreface sandstones (Pliocene to lowermost Pleistocene).

Identification of these potential reservoir rocks may have a strong impact on exploratory activities within the study area.

- Based on the integrated seismic sequence analysis, the S3, S6, S7, S9, S10 and S11 seismic sequences would conform regional seals whereas internal seals have been interpreted per each sequence described in the previous point. In addition, mass transport deposits (MTD, SF11) recognized on the western margin (S2-S4 sequences) and extending to Trujillo Basin, could be considered as seal rocks for the Eocene-Miocene turbiditic reservoirs due to their lithological composition. 
- Nine potential plays (series of traps) have been interpreted in the study area as very attractive exploratory targets that could qualify to a follow-up assessment. All the Cenozoic trap types in the study area, mostly controlled by extensional tectonics, have been formed during Miocene to lowermost Pleistocene, which is very favorable for the hydrocarbon preservation due to "critical moment" of petroleum system is proposed at ca. $3 \mathrm{Ma}$.

- The Pliocene to lowermost Pleistocene potential reservoir rocks could accumulate remigrated petroleum due to faulting and uplift generated during the last tectonic phase in the basin (S9-S11), correlated with Quechua IV Andean tectonic phase.

- The most Direct Hydrocarbon Indicators (DHIs) recognized in the study area are hosted within the Cenozoic succession, which adds new evidence of generated hydrocarbon in the basin and increase the exploratory potential for Cenozoic reservoirs.

- This work adds new potential reservoir and seal rocks for the petroleum systems previously defined in the basin: Upper Jurassic Chicama - Cenozoic (.) and Albian La Zorra - Cenozoic (.).

- Seismic sequence boundaries in the Cenozoic succession were developed in response to a variety of factors. These seismic surfaces are local to regional unconformities developed in response to tectonics, sea-level change and aseismic ridge subduction, e.g. SB2 is purely related to tectonic uplift (Incaic III tectonic phase) while SB9 is mostly related to Quechua IV tectonic phase and the sea-level rise decreasing.

- The main source areas for deposition of the sequences correspond to the PreCenozoic Basement rocks exposed onshore along the Andean Mountains, and the Paracas Arch when was uplifted and exposed during the basin evolution. 


\section{ACKNOWLEDGEMENTS}

The authors acknowledge SAVIA PERU S.A. and PERUPETRO for supplying the seismic and well data, and borehole samples respectively; which made the present study possible. Diego Timoteo thanks Brazilian agency CAPES for graduate Scholarship. 


\section{REFERENCES}

Aleman, A., Ramos, V.A., 2000. The Northern Andes. In: Cordani, U.J., Milani, E.J., Thomaz Filho, A., Campos, D.A. (Eds.), Tectonic Evolution of South America, 31 ${ }^{\circ}$ International Geological Congress, pp. 453-480. Río de Janeiro.

Azalgara, C., 1993. Structural evolution of the offshore forearc basins of Peru, including the Salaverry, Trujillo, Lima, West Pisco and East Pisco basins. A Thesis submitted in partial fulfillment of the requirements for the degree Master of Arts. Rice University Houston, Texas.

Benavides, V., 1999. Orogenic evolution of the Peruvian Andes: the Andean cycle. In: Skinner, B. (Ed.), Geology and Mineral Deposits of Central Andes. Society of Economic Geology, Special Publication, vol. 7, pp. 61-108.

Canchanya, Z., Paz, M., 1992. Estudio Bioestratigráfico en base a nannoplancton calcáreo: pozo Delfin-1X y Ballena-1X, Cuenca Salaverry. Petroperu, unidad de investigación y desarrollo. Reporte interno Petroperu.

Chierici, G.L., 1995. Principles of Petroleum Reservoir Engineering, Volume 1. Springer-Verlag Berlin Heidelberg GmbH. Pp. 419.

DeCelles P.G., Ducea M.N., Kapp P., Zandt G., 2009, Cyclicity in Cordilleran orogenic systems: Nature Geoscience, v. 2, p. 251-257, doi:10.1038/ngeo469.

Deckelman, J.A., Connors, F.X., Shultz, A.W., Glagola, P.A., Menard, W.M., Schwegal, S.R, Shearer, J.N., 2008. Neogene Oil And Gas Reservoirs in The Progreso Basin, Offshore Ecuador And Peru: Implications For Petroleum Exploration And Development. Journal of Petroleum Geology, vol. 31, pp. 43-60.

Dunbar, R.B, Marty, R.C., Baker, P.A., 1990. Cenozoic marine sedimentation in the Sechura and Pisco Basins, Peru. Palaeogeography, Palaeoclimatology, Palaeoecology, Vol. 41, pp. 235-261.

Gaffney-Cline, 2005. Interpretation of balanced and restored sections for the Trujillo, Salaverry and Pisco Basins, Offshore Peru. In: Sequence stratigraphic analysis of the Peruvian Offshore Continental Shelf-Slope. Appendix F. Final Report prepared for Perupetro S. A. by Gaffney, Cline \& Associates, Inc.

Galbraith, R.F., Laslett, G.M., 1993. Statistical models for mixed fission track ages. Nuclear Tracks and Radiation Measurements, no. 21, pp. 459-470.

Guevara, C., 1980. El Grupo Casma del Perú Central entre Trujillo y Mala. Bol. SGP, Vol. 67, pp. 73-83.

Gleadow, A. J. W., 1981. Fission-track dating methods: what are the real alternatives?. Nuclear Tracks 5, 3-14.

Gonzales, E., 2014. Biostratigraphy Chart Salaverry Basin - Chan Chan Area: based on microfossils (planktonic, benthonic, nanno, pollen and spores). Savia Peru S.A. Internal Report. 
Haq, B. U., Hardenbol, J., and Vail, P. R. (1987). Chronology of fluctuating sea levels since the Triassic (250 million years ago to present). Science, Vol. 235, pp. 1156-1166.

Hurford, A.J., Green, P.F., 1982. A user's guide to fission track dating calibration. Earth Planet. Sci. Lett.,59, 343-54.

Hurford, A.J., Green, P.F., 1983. The zeta age calibration of fission track dating. Chem. Geol. Isot. Geosci. Sect. 31, 285-317.

James, N.P. and Wood, R. 2010. Reefs, In: James Np.P. and Dalrymple, R.W., (eds) Facies Models 4. Geological Association of Canada, GEOtext 6, pp. 421-447.

Ketcham, R.A., 2005. Forward and inverse modeling of low-temperature thermochronometry data. Rev. Mineral. Geochem. 58 (1), 275-314.

Ketcham, R.A., Carter, A., Donelick, R.A., Barbarand, J., Hurford, A.J., 2007. Improved modeling of fission-track annealing in apatite. Am. Mineral. 92 (5-6), 799810 .

Kulm, L.D., Prince, R.A., French, W., Johnson, S., Masias, A., 1981. Crustal structure and tectonics of the central Perú continental margin and trench. Geological Society of America, Memoir 154, 445-508.

Martinez, E., Fernandez, J., Calderón, Y., Hermoza, W., Galdos, C., 2005. Tumbes and Talara Basins Hydrocarbon Evaluation. Exploration Department, Basin Evaluations Group. Perupetro Internal report.

Minguito, M.A., Machín, J., Cakebread, J., Beroiz, C., 1997. Hydrocarbon Potential Review. Z-29 block, Trujillo basin, Perú. Volume I: Geology. Repsol Exploración S.A. Internal Report.

Mitchum, R.M., Vail, P.R., Sangree, J.B., 1977. Seismic stratigraphy and global changes of sea level, Part 6: Stratigraphic interpretation of seismic reflection patterns in depositional sequences. In: Payton, C.E. (Ed.), Seismic Stratigraphy - Applications to Hydrocarbon Exploration. American Association of Petroleum Geologists, Memoir 26, pp. 117-135.

Morales, C., Gonzales, E., 2006. Determinación litológica y bioestrtigráfica de los pozos Ballena-1X, Delfin-1X, Lobos-1X y Morsa-1X, Cuenca Trujillo. Petro-tech Peruana Internal Report.

Myers, J.S., 1974. Cretaceous stratigraphy and structure, Western Andes of Peru between latitudes $10^{\circ}-10^{\circ} 30^{\prime \prime}$. American Association of Petroleum Geologists Bulletin $58(3), 474-487$.

Myers, J.S., 1980. Geología de los Cuadrángulos de Huarmey y Huayllapampa. Bol. INGEMMET. Boletin N³3, Serie A, Carta Geologica Nacional.

Narvaez, J., Pardo, A., 1993. Nueva fauna fósil del Eoceno del pozo Delfin-1X (Margen continental, norte del Perú): Alcances estratigráficos y tectónicos. Reporte interno Petroperu. 
Neal, J., and Abreu, V., 2009, Sequence stratigraphy hierarchy and the accommodation succession method, Geology, v. 37, p. 779-782

Okada, H., 1990. Nannofossils biostratigraphy of the Ballena-1X and Delfin-1X wells. In: Azalgara, C. (Msc Thesis), Structural Evolution of the Offshore Forearc Basins of Peru, Including the Salaverry, Trujillo, Lima, West Pisco and East Pisco Basins. Appendix A.

Peña, D., 2011. Geologia del Cenozoico - Cuenca Salaverry. Savia Peru Reporte Interno.

Pfiffner, O.A., and Gonzalez, L., 2013. Mesozoic-Cenozoic evolution of the western margin of South America: Case study of the Peruvian Andes. Geosciences, vol. 3, pp. 262-310, doi:10.3390/geosciences3020262.

Quesada, S., Ferrando, R. and Bottinga, R., 2000. Source Rock Characteristics, Maturity Modeling and Oil to source Correlation. Repsol Internal Report. Repsol YPF, Denver Technical Centre.

Ramos, V.A., 2009. Anatomy and global context of the Andes: main geologic features and the Andean orogenic cycle. In: Kay, S.M., Ramos, V.A., Dickinson, W. (Eds.), Backbone of the Americas: Shallow Subduction, Plateau Uplift, and Ridge and Terrane Collision, Memoir 204. Geological Society of America, pp. 31-65.

Ramos, V.A., 2014. Evolución Tectónica Paleozoica de los Andes Peruanos: Nuevos datos y nuevas hipótesis. Instituto de Estudios Andinos (Universidad de Buenos Aires CONICET). Conferencia Magistral, XVII Congreso Geológico Peruano, Lima-Perú.

Reitsma, M.J., 2012. Reconstructing the Late Paleozoic-Early Mesozoic plutonic and sedimentary record of South-East Peru : orphaned back-arcs along the Western Margin of Gondwana. Terre \& Environnement; Vol. 111. Thèse ; 4459. Thèse présentée à la Faculté des Sciences de l'Université de Genève pour obtenir le grade de Docteur ès sciences, mention Sciences de la Terre. https://archive-ouverte.unige.ch/unige:23095.

Romero, D., Valencia, K., Alarcón, P., Peña, D. and Ramos, V.A. 2013. The offshore basement of Perú: Evidence for different igneous and metamorphic domains in the Forearc. Journal of South American Earth Sciences, vol. 42, pp. 47-60.

Sangree, J.B., Widmier, J.M., 1977. Seismic stratigraphy and global changes of sea level, Part 9: Seismic interpretation of clastic depositional facies. In: Payton, C.E. (Ed.), Seismic Stratigraphy - Applications to Hydrocarbon Exploration. American Association of Petroleum Geologists, Memoir 26, pp. 165-184.

Schell, W. W., 1978. Paleontology Occidental Wells, Peru. Occidental Petroleum Company Internal Report.

Schrader, H., and Cruzado, J., 1990. The Ballena and Delfin wells off central Peru: revised ages. In Suess, E., von Huene, R., et al., Proc. ODP, Sci. Results, Vol. 112: College Station, TX (Ocean Drilling Program), pp. 209-215. http://dx.doi.org/10.2973/odp.proc.sr.112.187.1990.

Timoteo, D., Romero, D., and Valencia, K., 2012. Posible existencia de la Faja Alleghanide? en el Noroeste del Perú: Recientes dataciones $\mathrm{Ar} / \mathrm{Ar}$ y U-Pb, e 
implicancias en un Sistema Petrolero de la Cuenca Talara. Sociedad Geológica del Perú. XVI Congreso Peruano de Geología - Lima, Perú. DOI: 10.13140/RG.2.1.4571.1207.

Timoteo, D., 2013. Potencial Generador de Hidrocarburos y Distribución de las Secuencias Cretácicas costa afuera (offshore) del Perú centro-norte (Lima-Chiclayo). Tesis para obtar el Título Profesional de Ingeniero Geólogo. UNI, Lima- Perú.

Timoteo, D., and Valencia, K., 2014. Caracterização Geológica-Geoquímica das rochas geradoras Cretácicas potenciais da Bacia Salaverry, Peru. Anais do 47 Congresso Brasileiro de Geologia - Salvador - BA - 2014, pp. 1485.

Vail, P.R., Mitchum, R.M., Thompson, S., 1977. Seismic stratigraphy and global changes of sea level, Part 3: Relative changes of sea level from coastal onlap. In: Payton, C.E. (Ed.), Seismic Stratigraphy - Applications to Hydrocarbon Exploration. American Association of Petroleum Geologists, Memoir 26, pp. 83-98.

Vail, P. R. 1987. Seismic Stratigraphy Interpretation Using Sequence Stratigraphy: Part 1: Seismic Stratigraphy Interpretation Procedure. In Atlas of Seismic Stratigraphy (A. W. Bally, Ed.), pp. 1-10. American Association of Petroleum Geologists Studies in Geology No 27.

Valdespino, T., Seminario, F., 1976a. Informe Micropaleontológico del pozo Ballena8X-1. Reporte interno Petroperu.

Valdespino, T., Seminario, F., 1976b. Informe Micropaleontológico del pozo Delfin 20X-1X. Reporte interno Petroperu.

Valencia, K., and Romero, D., 2011. Evolución Tectono-sedimentaria de la Cuenca Salaverry y su Implicancia en la Generación de Hidrocarburos. VII Seminario Internacional de Exploración y Producción de Petróleo y Gas - INGEPET Perupetro S.A. Lima- Perú.

Valencia, K., Romero, D., Alarcón, P., Llerena, C., Borda, E., and Rojas, J., 2013. Structural Evolution of Convergent Margin of Central Peru and its Relationship with Petroleum Systems of the Forearc Salaverry Basin. AAPG Search and Discovery Article \#90166.

Veeken, P.C., 2013. Seismic Stratigraphy and Depositional Facies Models. EAGE Publications, pp. 467.

Wine, G., Arcuri, J., Martínez, E., Monges, C., Calderón, Y., Galdos, C., 2001. Hydrocarbon Potential of the Salaverry Offshore Basin Peru. Perupetro Internal Report. 


\section{CAPÍTULO 4: CONCLUSÕES}

- A sucessão Cenozóica na porção central da Bacia Salaverry é subdividida em onze sequências sísmicas: 3 sequências de segunda ordem (S1, S2 e S5) e 8 sequências de terceira ordem (S3, S4, S6-S11). Todas as sequências são delimitadas por doze limites de sequências.

- Análises termocronológicas por traços de fissão em apatita permitiram definir no registro da bacia um evento de soerguimento principal em torno de 25 Ma que correlaciona com um forte rebaixamento do nível do mar durante o Oligoceno, os quuais em conjunto provocaram a erosão das rochas Oligocênicas e a secção superior dos depósitos do Eoceno superior. Esse evento de soerguimento é correlacionado à fase tectônica Incaica III.

- Dentro das sequências sísmicas S1, S2, S4, S5, S7 e S8 tem sido interpretados sistemas deposicionais chaves que poderiam conter reservatórios potencias:

S1: Arenitos deltaicos/marinho-rasos (Eoceno superior).

S2: Arenitos deltaicos e do tipo flysch (Mioceno inferior).

S4: Arenitos turbidíticos relacionados com depósitos pelágicos/hemipelágicos (Mioceno médio).

S5: Arenitos turbidíticos relacionados com depósitos pelágicos/hemipelágicos (Mioceno médio).

S7. Recifes de carbonato (Plioceno).

S8: Arenitos de lobos turbidíticos e arenitos deltaicos/marinho-rasos

A identificação dessas rochas reservatório potenciais podem ter um forte impacto sobre as atividades exploratórias na área de estudo.

- Com base na análise integrada da sequência sísmica, as sequências S3, S6, S7, S9, S10 e S11 poderiam conformar selantes regionais enquanto as selantes internas têm sido interpretadas por cada sequência descrita no ponto anterior. Além disso, os depósitos de transporte de massa (SF11) reconhecidos na margem ocidental e que se estendem para a Bacia Trujillo poderiam ser considerados como rochas selantes para 
os reservatórios turbidíticos de idade Eoceno-Mioceno, devido a sua composição litológica.

- Nove plays (série de armadilhas) potenciais têm sido interpretados na área de estudo como alvos exploratórios muito atraentes que poderiam qualificar para uma avaliação de detalhe. Todos os tipos de armadilhas Cenozóicas na área de estudo, principalmente controlados por tectônica extensional, foram formados a partir do Mioceno até o Pleistoceno inferior, o qual é muito favorável para a preservação de hidrocarbonetos devido a que o momento crítico do sistema petrolífero é proposto em torno de $3 \mathrm{Ma}$.

- Os reservatórios potenciais de idade Plioceno-Pleistoceno poderiam conter petróleo remigrado devido ao falhamento e soerguimento gerado durante a ultima fase tectônica na bacia (S9-S11), correlacionado com a fase tectônica andina Quechua IV.

- A maioria dos Indicadores Diretos de Hidrocarbonetos (DHIs) reconhecidos na área de estudo se encontram dentro da sucessão Cenozóica, o que adiciona novas evidências de hidrocarbonetos gerados na bacia e acrescenta o potencial exploratório dos reservatórios Cenozóicos.

- A presente dissertação adiciona novas rochas reservatórios e rochas selantes potenciais para os sistemas petrolíferos previamente definidos na bacia.

- Os limites de sequência na sucessão Cenozóica foram desenvolvidos em resposta a uma variedade de fatores. Essas superfícies sísmicas são discordâncias locais e regionais desenvolvidas em resposta à tectônica, às variações do nível do mar e à subducção da dorsal de Nazca. Por exemplo, o limite de sequência SB2 é puramente relacionado ao soerguimento tectônico (fase tectônica Incaica III) enquanto o limite de sequência SB9 é principalmente relacionado à fase tectônica Quechua IV e também à diminuição da subida do nível do mar. 
- As principais áreas fonte para a deposição das sequências correspondem às rochas que compõem o embasamento Pré-Cenozóico expostas ao longo das montanhas Andinas, e o Arco de Paracas quando foi elevado e exposto durante a evolução da bacia. 


\section{REFERÊNCIAS BIBLIOGRÁFICAS}

Aleman, A., Ramos, V.A. 2000. The Northern Andes. In: Cordani, U.J., Milani, E.J., Thomaz Filho, A., Campos, D.A. (Eds.), Tectonic Evolution of South America, $31^{\circ}$ International Geological Congress, pp. 453-480. Río de Janeiro.

Alemán, A. 2006. Collision of aseismic ridges in Perú. Backbone of the Americas Symposium, Geological Society of America and Asociación Geológica Argentina, Abstracts with Programs, Special Meeting 2, Mendoza, p. 21.

Azalgara, C. 1993. Structural evolution of the offshore forearc basins of Peru, including the Salaverry, Trujillo, Lima, West Pisco and East Pisco basins. A Thesis submitted in partial fulfillment of the requirements for the degree Master of Arts. Rice University - Houston, Texas.

Baby, P., Calderon, Y, Louterbach, M., Eude, A., Espurt, N., Brusset, S., Roddaz, M., Brichau, S., Calves, G., Hurtado, C., Ramirez, L., Quispe, A., Bolaños, R. 2014. Thrusts propagation and new geochronologic constraints in the Peruvian subandean Fold and Thrust Belt. VIII Seminario Internacional de Exploración y Producción de Petróleo y Gas - INGEPET Perupetro S.A. Lima- Perú.

Benavides, V. 1999. Orogenic evolution of the Peruvian Andes: the Andean cycle. In: Skinner, B. (Ed.), Geology and Mineral Deposits of Central Andes. Society of Economic Geology, Special Publication, vol. 7, pp. 61-108.

Canchanya, Z., Paz, M. 1992. Estudio Bioestratigráfico en base a nannoplancton calcareo: pozo Delfin-1X y Ballena-1X, Cuenca Salaverry. Petroperu, unidad de investigación y desarrollo. Reporte interno Petroperu.

Castroviejo, R., Rodrigues, J., Tassinari, C.G., Willner, A. 2014. Ophiolites in the Peruvian Andes: preliminary data. Abstract. In: LAK 2011: 22th Colloquium on Latin American Earth Sciences, Proceedings, 11-14 July, 2011, Lisbon, Portugal.

Catuneanu et. al., 2011. Sequence stratigraphy: methodology and nomenclature. Newsletters on Stratigraphy, Vol. 44/3, pp.173-245.

Chierici, G.L. 1995. Principles of Petroleum Reservoir Engineering, Volume 1. Springer-Verlag Berlin Heidelberg GmbH. Pp. 419.

DeCelles P.G., Ducea M.N., Kapp P., Zandt G. 2009, Cyclicity in Cordilleran orogenic systems: Nature Geoscience, v. 2, p. 251-257, doi:10.1038/ngeo469. 
Deckelman, J.A., Connors, F.X., Shultz, A.W., Glagola, P.A., Menard, W.M., Schwegal, S.R, Shearer, J.N. 2008. Neogene Oil and Gas Reservoirs in the Progreso Basin, Offshore Ecuador And Peru: Implications for Petroleum Exploration and Development. Journal of Petroleum Geology, vol. 31, pp. 4360.

Dickinson, W.F., and Snyder, W.S. 1979. Geometry of subducted slabs related to San Andres transform: The Journal of Geology, vol. 88, pp. 619-638.

Donelick, R.A., O’Suillivan. P.B., Ketcham, R.A. 2005. Apatite Fission-Track Analysis. Reviews in Mineralogy \& Geochemistry. Vol. 58, pp. 49-94, 2005.

Dunbar, R.B, Marty, R.C., Baker, P.A. 1990. Cenozoic marine sedimentation in the Sechura and Pisco Basins, Peru. Palaeogeography, Palaeoclimatology, Palaeoecology, Vol. 41, pp. 235-261.

Eiras, J., Filho, J. 2003. Sistemas Petrolíferos ígneo-sedimentares. 2o Congresso Brasileiro de P\&D em Petróleo \& Gas. Resumos Estendidos.

Espurt N., Baby P., Brusset S., Roddaz M., Hermoza W., Regard V., Antoine P.O., Salas-Gismondi R., Bolaños R. 2007. Influence of the Nazca Ridge subduction on the modern Amazonian retro-foreland basin, Geology, Vol. 35, No. 6: 515518.

Gaffney-Cline. 2005. Interpretation of balanced and restored sections for the Trujillo, Salaverry and Pisco Basins, Offshore Peru. In: Sequence stratigraphic analysis of the Peruvian Offshore Continental Shelf-Slope. Appendix F. Final Report prepared for Perupetro S. A. by Gaffney, Cline \& Associates, Inc.

Galbraith, R.F., Laslett, G.M. 1993. Statistical models for mixed fission track ages. Nuclear Tracks and Radiation Measurements, no. 21, pp. 459-470.

Guevara, C. 1980. El Grupo Casma del Perú Central entre Trujillo y Mala. Bol. SGP, Vol. 67, pp. 73-83.

Gleadow, A. J. W. 1981. Fission-track dating methods: what are the real alternatives?. Nuclear Tracks 5, 3-14.

Gonzales, E. 2014. Biostratigraphy Chart Salaverry Basin - Chan Chan Area: based on microfossils (planktonic, benthonic, nanno, pollen and spores). Savia Peru S.A. Internal Report. 
Hampel, A. 2002, The migration history of the Nazca Ridge along the Peruvian active margin: A re-evaluation: Earth and Planetary Science Letters, v. 203, p. 665679, doi: 10.1016/ S0012-821X(02)00859-2.

Haq, B. U., Hardenbol, J., and Vail, P. R. 1987. Chronology of fluctuating sea levels since the Triassic (250 million years ago to present). Science, Vol. 235, pp. $1156-1166$.

Hurford, A.J., Green, P.F. 1982. A user's guide to fission track dating calibration. Earth Planet. Sci. Lett.,59, 343-54.

Hurford, A.J., Green, P.F. 1983. The zeta age calibration of fission track dating. Chem. Geol. Isot. Geosci. Sect. 31, 285-317.

James, N.P. and Wood, R. 2010. Reefs, In: James, N.P. and Dalrymple, R.W., (eds) Facies Models 4. Geological Association of Canada, GEOtext 6, pp. 421-447.

Kay, S. and Coira, B. 2009. Shallowing and steepening subduction zones, continental lithospheric loss, magmatism, and crustal flow under the Central Andean Altiplano-Puna Plateau. In: Kay, S., Ramos, V. \& Dickinson, W. (eds) Backbone of the Americas: Shallow Subdcution Plateau Uplift and Ridge and Terrane Collision. Geological Society of America Memoirs, vol. 204, pp. 229 -259, doi: 10.1130/2009.1204(11).

Ketcham, R.A. 2005. Forward and inverse modeling of low-temperature thermochronometry data. Rev. Mineral. Geochem. 58 (1), 275-314.

Ketcham, R.A. Carter, A., Donelick, R.A., Barbarand, J., Hurford, A.J., 2007. Improved modeling of fission-track annealing in apatite. Am. Mineral. 92 (5-6), 799-810.

Kulm, L.D., Prince, R.A., French, W., Johnson, S., Masias, A. 1981. Crustal structure and tectonics of the central Perú continental margin and trench. Geological Society of America, Memoir 154, 445-508.

Lajo, A. 2014. Modelagem de Bacias e Sistemas Petrolíferos 2D do Setor Ilha Lobos de Afuera - Chiclayo: Bacias Sechura e Trujillo, Noroeste Offshore do Peru. Dissertação de Mestrado em Engenharia Civil - COPPE/UFRJ.

Martinez, E., Fernandez, J., Calderón, Y., Hermoza, W., Galdos, C. 2005. Tumbes and Talara Basins Hydrocarbon Evaluation. Exploration Department, Basin Evaluations Group. Perupetro Internal report.

Minguito, M.A., Machín, J., Cakebread, J., Beroiz, C. 1997. Hydrocarbon Potential Review. Z-29 block, Trujillo basin, Perú. Volume I: Geology. Repsol Exploración S.A. Internal Report. 
Mitchum, R.M., Vail, P.R., Sangree, J.B. 1977. Seismic stratigraphy and global changes of sea level, Part 6: Stratigraphic interpretation of seismic reflection patterns in depositional sequences. In: Payton, C.E. (Ed.), Seismic Stratigraphy Applications to Hydrocarbon Exploration. American Association of Petroleum Geologists, Memoir 26, pp. 117-135.

Montgomery, D.R., Balco, G., Willett, S.D. 2001. Climate, tectonics, and the morphology of the Andes: Geology, vol. 29, pp. 579-582, doi: 10.1130/00917613(2001)029<0579:CTATMO>2.0.CO;2.

Morales, C., Gonzales, E. 2006. Determinación litológica y bioestrtigráfica de los pozos Ballena-1X, Delfin-1X, Lobos-1X y Morsa-1X, Cuenca Trujillo. Petro-tech Peruana Internal Report.

Myers, J.S. 1974. Cretaceous stratigraphy and structure, Western Andes of Peru between latitudes $10^{\circ}-10^{\circ} 30^{\prime \prime}$. American Association of Petroleum Geologists Bulletin 58 (3), 474-487.

Myers, J.S. 1980. Geología de los Cuadrángulos de Huarmey y Huayllapampa. Bol. INGEMMET. Boletin N³3, Serie A, Carta Geologica Nacional.

Narvaez, J., Pardo, A. 1993. Nueva fauna fósil del Eoceno del pozo Delfin-1X (Margen continental, norte del Perú): Alcances estratigráficos y tectónicos. Reporte interno Petroperu.

Neal, J., and Abreu, V. 2009, Sequence stratigraphy hierarchy and the accommodation succession method, Geology, v. 37, p. 779-782.

Okada, H. 1990. Nannofossils biostratigraphy of the Ballena-1X and Delfin-1X wells. In: Azalgara, C. (Msc Thesis), Structural Evolution of the Offshore Forearc Basins of Peru, Including the Salaverry, Trujillo, Lima, West Pisco and East Pisco Basins. Appendix A.

Pardo Casas, F., and Molnar, P. 1987. Relative motion of the Nazca (Farallon) and South American plates since Late Cretaceous time. Tectonics, vol. 6, pp. 233248, doi: 10.1029/TC006i003p00233.

Peña, D. 2011. Geologia del Cenozóico - Cuenca Salaverry. Savia Peru Reporte Interno.

Pfiffner, O.A., and Gonzalez, L. 2013. Mesozoic-Cenozoic evolution of the western margin of South America: Case study of the Peruvian Andes. Geosciences, vol. 3, pp. 262-310, doi:10.3390/geosciences3020262. 
Quesada, S., Ferrando, R. and Bottinga, R. 2000. Source Rock Characteristics, Maturity Modeling and Oil to source Correlation. Repsol Internal Report. Repsol YPF, Denver Technical Centre.

Ramos, V.A. 2008. The basement of the Central Andes: The Arequipa and related terranes: Annual Review of Earth and Planetary Sciences, v. 36, p. 289-324, doi: 10.1146/annurev.earth.36.031207.124304.

Ramos, V.A. 2009a. Evolución de Los Andes Centrales. Laboratorio de Tectónica Andina. Curso in-house Petro-tech Peruana S.A.

Ramos, V.A. 2009b. Anatomy and global context of the Andes: main geologic features and the Andean orogenic cycle. In: Kay, S.M., Ramos, V.A., Dickinson, W. (Eds.), Backbone of the Americas: Shallow Subduction, Plateau Uplift, and Ridge and Terrane Collision, Memoir 204. Geological Society of America, pp. $31-65$.

Ramos, V.A. 2010. The Tectonic Regime along the Andes: present settings as a key for the Mesozoic regimes. Geological Journal, vol. 45, pp. 2-25.

Ramos, V.A. 2014. Evolución Tectónica Paleozóica de los Andes Peruanos: Nuevos datos y nuevas hipótesis. Instituto de Estudios Andinos (Universidad de Buenos Aires - CONICET). Conferencia Magistral, XVII Congreso Geológico Peruano, Lima-Perú.

Ramos, V.A., and Folguera, A. 2009, Andean flat slab subduction through time, in Murphy, B., ed., Ancient Orogens and Modern Analogues, The Geological Society, Special Publication 327: 31-54, London.

Reitsma, M.J. 2012. Reconstructing the Late Paleozoic-Early Mesozoic plutonic and sedimentary record of South-East Peru : orphaned back-arcs along the Western Margin of Gondwana. Terre \& Environnement ; Vol. 111. Thèse ; 4459. Thèse présentée à la Faculté des Sciences de l'Université de Genève pour obtenir le grade de Docteur ès sciences, mention Sciences de la Terre. https://archiveouverte.unige.ch/unige:23095.

Romero, D., Valencia, K., Alarcón, P., Peña, D. and Ramos, V.A. 2013. The offshore basement of Perú: Evidence for different igneous and metamorphic domains in the Forearc. Journal of South American Earth Sciences, vol. 42, pp. 47-60.

Sangree, J.B., Widmier, J.M. 1977. Seismic stratigraphy and global changes of sea level, Part 9: Seismic interpretation of clastic depositional facies. In: Payton, 
C.E. (Ed.), Seismic Stratigraphy - Applications to Hydrocarbon Exploration. American Association of Petroleum Geologists, Memoir 26, pp. 165-184.

Schell, W.W. 1978. Paleontology Occidental Wells, Peru. Occidental Petroleum Company Internal Report.

Schrader, H., and Cruzado, J. 1990. The Ballena and Delfin wells off central Peru: revised ages. In Suess, E., von Huene, R., et al., Proc. ODP, Sci. Results, Vol. 112: College Station, TX (Ocean Drilling Program), pp. 209-215. http://dx.doi.org/10.2973/odp.proc.sr.112.187.1990.

Shell Oil Company, 1987. Facies Analysis of a Depositional/Seismic Sequence. In: Bally, A. W. (Ed.). Atlas of Seismic Stratigraphy. AAPG. Studies in Geology (27), p. 37-52.

Somoza, R. 1998. Updated Nazca (Farallon)-South America relative motions during the last 40 My: Implications for mountain building in the Central Andean region: Journal of South American Earth Sciences, vol. 11, pp. 211-215, doi: 10.1016/S0895-9811(98)00012-1.

Tassinari, C.G., Castroviejo, R., Rodrigues, J.F., Acosta, J., Pereira, E. 2011. A Neoproterozoic age for the chromitite and gabbro of the Tapo ultramafic Massif, Eastern Cordillera, Central Peru and its tectonic implications. Journal of South American Earth Sciences, vol 32, pp. 429-437.

Timoteo, D., Romero, D., and Valencia, K. 2012. Posible existencia de la Faja Alleghanide? en el Noroeste del Perú: Recientes dataciones Ar/Ar y U-Pb, e implicancias en un Sistema Petrolero de la Cuenca Talara. Sociedad Geológica del Perú. XVI Congreso Peruano de Geología - Lima, Perú. DOI: 10.13140/RG.2.1.4571.1207.

Timoteo, D. 2013. Potencial Generador de Hidrocarburos y Distribución de las Secuencias Cretácicas costa afuera (offshore) del Perú centro-norte (Lima Chiclayo). Tesis para obtar el Título Profesional de Ingeniero Geólogo. UNI, Lima- Perú.

Timoteo, D., and Valencia, K. 2014. Caracterização Geológica-Geoquímica das rochas geradoras Cretácicas potenciais da Bacia Salaverry, Peru. Anais do 47 Congresso Brasileiro de Geologia - Salvador - BA - 2014, pp. 1485.

Vail, P.R., Mitchum, R.M., Thompson, S. 1977. Seismic stratigraphy and global changes of sea level, Part 3: Relative changes of sea level from coastal onlap. In: Payton, C.E. (Ed.), Seismic Stratigraphy - Applications to Hydrocarbon Exploration. American Association of Petroleum Geologists, Memoir 26, pp. 83-98. 
Vail, P.R. 1987. Seismic Stratigraphy Interpretation Using Sequence Stratigraphy: Part 1: Seismic Stratigraphy Interpretation Procedure. In Atlas of Seismic Stratigraphy (A. W. Bally, Ed.), pp. 1-10. American Association of Petroleum Geologists Studies in Geology No 27.

Vail et. al. 1991. An integrated approach to exploration and development in the 90s: well log-seismic sequence stratigraphy analysis: Gulf Coast Association of Geological Societies Transactions, v. 41, p. 630-650.

Valdespino, T., Seminario, F. 1976a. Informe Micropaleontológico del pozo Ballena $8 \mathrm{X}-1$. Reporte interno Petroperu.

Valdespino, T., Seminario, F. 1976b. Informe Micropaleontológico del pozo Delfin 20X-1X. Reporte interno Petroperu.

Valencia, K., and Romero, D. 2011. Evolución Tectono-sedimentaria de la Cuenca Salaverry y su Implicancia en la Generación de Hidrocarburos. VII Seminario Internacional de Exploración y Producción de Petróleo y Gas - INGEPET Perupetro S.A. Lima- Perú.

Valencia, K., Romero, D., Alarcón, P., Llerena, C., Borda, E., and Rojas, J. 2013. Structural Evolution of Convergent Margin of Central Peru and its Relationship with Petroleum Systems of the Forearc Salaverry Basin. AAPG Search and Discovery Article \#90166.

Veeken, P.C. 2013. Seismic Stratigraphy and Depositional Facies Models. EAGE Publications, pp. 467.

Web1. 2013. http://m.larepublica.pe/ultimas/1009670/en-octubre-adjudicaran-los-9lotes-frente-a-la-costa-y-habria-us-450-mlls-de-inversion.

Web2. 2013. http://www.bloomberg.com/news/articles/2013-03-25/exxon-apachelooking-to-bid-on-peru-s-offshore-oil-gas-blocks.

Willner A.P., Castroviejo, R., Rodrigues, J.F., Acosta, J., Rivera, M. 2010. High pressure metamorphic conditions in garnet amphibolite from a collisional shear zone related to the Tapo ultramafic body, Eastern Cordillera of central Perú. XV Congreso Peruano de Geología, Resúmenes Extendidos, Sociedad Geológica del Perú, Publicación Especial 9, pp. 87-90, Cusco.

Wine, G., Arcuri, J., Martínez, E., Monges, C., Calderón, Y., Galdos, C. 2001. Hydrocarbon Potential of the Salaverry Offshore Basin Peru. Perupetro Internal Report. 
Wipf, M. 2006. Evolution of the Western Cordillera and Coastal Margin of Peru: Evidence from low temperature Thermochronology and Geomorphology. Swiss Federal Institute of Technology Zürich. PhD Thesis, 163 pp.

Zou, C. 2013. Volcanic Reservoirs in Petroleum Exploration. Pp.204. 


\section{ANEXO 1}

\section{CARTA DE SUBMISSÃO DO ARTIGO}




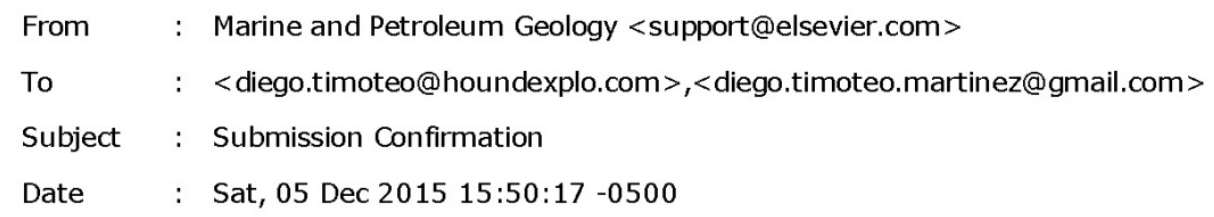

Article Type: Full Length Article.

Dear Mr. Diego Timoteo,

We have received your article "Tectono-sedimentary evolution of the Salaverry Basin, Peruvian Andes Forearc: new insights in assessing potential reservoirs and plays" for consideration for publication in Marine and Petroleum Geology.

Your manuscript will be given a reference number once an editor has been assigned.

To track the status of your paper, please do the following:

1. Go to this URL: http://ees.elsevier.com/jmpg/

2. Enter these login details:

Your username is: diego.timoteo@houndexplo.com

Your password is: *******

3. Click [Author Login]

This takes you to the Author Main Menu.

4. Click [Submissions Being Processed]

Thank you for submitting your work to this journal.

Kind regards,

Elsevier Editorial System

Marine and Petroleum Geology

$* * * * * * * * * * * * * * * * * * * * * * * * * * * * * * * * * * * * * * * * * * *$

Please note that the editorial process varies considerably from journal to journal. To view a sample editorial process, please click here:

http://ees.elsevier.com/eeshelp/sample_editorial_process.pdf

For further assistance, please visit our customer support site at

http://help.elsevier.com/app/answers/list/p/7923. Here you can search for solutions on a range of topics, find answers to frequently asked questions and learn more about EES via interactive tutorials. You will also find our 24/7 support contact details should you need any further assistance from one of our customer support representatives. 